\title{
Rayleigh Scattering at Atoms with Dynamical Nuclei
}

\section{Journal Article}

\section{Author(s):}

Fröhlich, J.; Griesemer, M.; Schlein, B.

Publication date:

2007

Permanent link:

https://doi.org/10.3929/ethz-b-000000345

Rights / license:

In Copyright - Non-Commercial Use Permitted

Originally published in:

Communications in Mathematical Physics 271(2), https://doi.org/10.1007/s00220-006-0134-x 


\title{
Rayleigh Scattering at Atoms with Dynamical Nuclei
}

\author{
J. Fröhlich ${ }^{1, \star}$, M. Griesemer ${ }^{2, \star \star}$, B. Schlein ${ }^{3, \star \star \star}$ \\ 1 Theoretical Physics, ETH-Hönggerberg, CH-8093 Zürich, Switzerland. \\ E-mail: juerg@itp.phys.ethz.ch \\ 2 Fachbereich Mathematik, Universität Stuttgart, D-70569 Stuttgart, Germany. \\ E-mail: marcel@mathematik.uni-stuttgart.de \\ 3 Department of Mathematics, Harvard University, Cambridge, MA 02138, USA. \\ E-mail: schlein@math.ucdavis.edu
}

Received: 6 September 2005 / Accepted: 2 June 2006

Published online: 8 February 2007 - @ C Springer-Verlag 2007

\begin{abstract}
Scattering of photons at an atom with a dynamical nucleus is studied on the subspace of states of the system with a total energy below the threshold for ionization of the atom (Rayleigh scattering). The kinematics of the electron and the nucleus is chosen to be non-relativistic, and their spins are neglected. In a simplified model of a hydrogen atom or a one-electron ion interacting with the quantized radiation field in which the helicity of photons is neglected and the interactions between photons and the electron and nucleus are turned off at very high photon energies and at photon energies below an arbitrarily small, but fixed energy (infrared cutoff), asymptotic completeness of Rayleigh scattering is established rigorously. On the way towards proving this result, it is shown that, after coupling the electron and the nucleus to the photons, the atom still has a stable ground state, provided its center of mass velocity is smaller than the velocity of light; but its excited states are turned into resonances. The proof of asymptotic completeness then follows from extensions of a positive commutator method and of propagation estimates for the atom and the photons developed in previous papers.

The methods developed in this paper can be extended to more realistic models. It is, however, not known, at present, how to remove the infrared cutoff.
\end{abstract}

\section{Introduction}

During the past decade, there have been important advances in our understanding of the mathematical foundations of quantum electrodynamics with non-relativistic, quantummechanical matter ("non-relativistic QED"). Subtle spectral properties of the Hamiltonians generating the time evolution of atoms and molecules interacting with the quantized radiation field have been established. In particular, existence of atomic ground states

\footnotetext{
* Activities supported, in part, by a grant from the Swiss National Foundation.

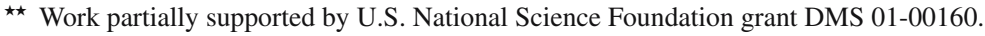

$\star \star \star$ Supported by a NSF postdoctoral fellowship
} 
and absence of stable excited states have been proven, and the energies and life times of resonances have been calculated in a rigorously controlled way, for a variety of models; see [BFS98, BFSS99, HS95, Sk98, GLL01, LL03, Gr04, AGG05, BFP05], and references given there. Furthermore, some important steps towards developing the scattering theory for systems of non-relativistic matter interacting with massless bosons, in particular photons, have been taken. Asymptotic electromagnetic field operators have been constructed in [FGS00], and wave operators for Compton scattering have been shown to exist in [Pi03]. Rayleigh scattering, i.e., the scattering of photons at atoms below their ionization threshold, has been analyzed in [FGS02] for models with an infrared cutoff. The results in this paper are based on methods developed in [DG99]. Some earlier results on Rayleigh scattering have been derived in [Sp97]. Compton scattering in models with an infrared cutoff has been studied in [FGS04]. While it is understood how to calculate scattering amplitudes for various low-energy scattering processes in models without an infrared cutoff, all known general methods to prove unitarity of the scattering matrix on subspaces of states of sufficiently low energy, i.e., asymptotic completeness, require the presence of an (arbitrarily small, but positive) infrared cutoff. In [Sp97, DG99, FGS02], Rayleigh scattering has only been studied for models of atoms with static (i.e., infinitely heavy) nuclei.

As suggested by this discussion, the main challenge in the scattering theory for nonrelativistic QED presently consists in solving the following problems:

i) To remove the infrared cutoff in the analysis of Rayleigh scattering;

ii) to remove the infrared cutoff in the treatment of Compton scattering of photons at one freely moving electron or ion;

iii) to prove asymptotic completeness of Rayleigh scattering of photons at atoms or molecules with dynamical nuclei.

In this paper, we solve problem iii) in the presence of an (arbitrarily small, but positive) infrared cutoff. In order to render our analysis, which is quite technical, as simple and transparent as possible, we consider the simplest model exhibiting all typical features and challenges encountered in an analysis of problem iii). We consider a hydrogen atom or a one-electron ion. The nucleus is described, like the electron, as a non-relativistic quantum-mechanical point particle of finite mass. We ignore the spin degrees of freedom of the electron and the nucleus. The electron and the nucleus interact with each other through an attractive two-body potential $V$, which can be chosen to be the electrostatic Coulomb potential. Electron and nucleus are coupled to a quantized radiation field. As in [FGS02], the field quanta of the radiation field are massless bosons, which we will call photons. Physically, the radiation field is the quantized electromagnetic field. However, the helicity of the photons does not play an interesting role in our analysis, and we therefore consider scalar bosons.

As announced, we focus our attention on Rayleigh scattering; we only consider the asymptotic dynamics of states of the atom and the radiation field with energies below the threshold for break-up of the atom into a freely moving nucleus and electron, i.e., below the ionization threshold. Moreover, we introduce an infrared cutoff: Photons with an energy below a certain arbitrarily small, but positive threshold energy do not interact with the nucleus and the electron. While all our other simplifications are purely cosmetic, the presence of an infrared cutoff is crucial in our proof of asymptotic completeness (but not for most other results presented in this paper).

In the model we study, the atom can be located anywhere in physical space and can move around freely, and the Hamiltonian of the system is translation-invariant. This feature suggests to combine and extend the techniques in two previous papers, [FGS02] 
(Rayleigh scattering with static nuclei) and [FGS04] (Compton scattering of photons at freely moving electrons), and this is, in fact, the strategy followed in the present paper. As in [FGS04], to prove asymptotic completeness we must impose an upper bound on the energy of the state of the system that guarantees that the center of mass velocity of the atom does not exceed one third of the velocity of light. This is a purely technical restriction which we believe can be replaced by one that guarantees that the velocity of the atom is less than the velocity of light. We note that, for realistic atoms, the condition that the total energy of a state be below the ionization threshold of an atom at rest automatically guarantees that the speed of the atom in an arbitrary internal state is much less than a third of the speed of light.

Next, we describe the model studied in this paper more explicitly. The Hilbert space of pure states is given by

$$
\mathcal{H}=L^{2}\left(\mathbb{R}^{3}, \mathrm{~d} x_{n}\right) \otimes L^{2}\left(\mathbb{R}^{3}, \mathrm{~d} x_{e}\right) \otimes \mathcal{F}
$$

where the variables $x_{n}$ and $x_{e}$ are the positions of the nucleus and of the electron, respectively, and $\mathcal{F}$ is the symmetric Fock space over the one-photon Hilbert space $L^{2}\left(\mathbb{R}^{3}, \mathrm{~d} k\right)$, where the variable $k$ denotes the momentum of a photon. Vectors in $\mathcal{F}$ describe pure states of the radiation field.

The Hamiltonian generating the time evolution of states of the system is given by

$$
H_{g}=H_{\mathrm{atom}}+H_{f}+g\left(\phi\left(G_{x_{e}}^{e}\right)+\phi\left(G_{x_{n}}^{n}\right)\right),
$$

where

$$
H_{\text {atom }}=\frac{p_{e}^{2}}{2 m_{e}}+\frac{p_{n}^{2}}{2 m_{n}}+V\left(x_{e}-x_{n}\right)
$$

is the Hamiltonian of the atom decoupled from the radiation field. Here $p_{e}=-i \nabla_{x_{e}}$ and $p_{n}=-i \nabla_{x_{n}}$ are the momentum operators of the electron and the nucleus, respectively, and $m_{e}$ and $m_{n}$ are their masses. The term $V\left(x_{e}-x_{n}\right)$ is the potential of an attractive twobody force, e.g., the electrostatic Coulomb force, between the electron and the nucleus; $(V(x)$ is negative and tends to zero, as $|x| \rightarrow \infty$, and it is assumed to be such that the spectrum of $H_{\text {atom }}$ is bounded from below and has at least one negative eigenvalue). The operator $H_{f}$ on the r.h.s. of (2) is the Hamiltonian of the free radiation field. It is given by

$$
H_{f}=\int \mathrm{d} k|k| a^{*}(k) a(k),
$$

where $|k|$ is the energy of a photon with momentum $k$, and $a^{*}(k), a(k)$ are the usual boson creation- and annihilation operators: For every function $f \in L^{2}\left(\mathbb{R}^{3}, \mathrm{~d} k\right)$,

$$
a^{*}(f)=\int \mathrm{d} k f(k) a^{*}(k) \quad \text { and } \quad a(f)=\int \mathrm{d} k \overline{f(k)} a(k)
$$

are densely defined, closed operators on the Fock space $\mathcal{F}$, and, for $f, h, \in L^{2}\left(\mathbb{R}^{3}, \mathrm{~d} k\right)$, they satisfy the canonical commutation relations

$$
\left[a(f), a^{*}(h)\right]=(f, h), \quad\left[a^{\sharp}(f), a^{\sharp}(h)\right]=0,
$$


where $(f, h)$ denotes the scalar product of $f$ and $h$. For $f \in L^{2}\left(\mathbb{R}^{3}, \mathrm{~d} k\right)$, a field operator $\phi(f)$ is defined by

$$
\phi(f)=a(f)+a^{*}(f) .
$$

It is a densely defined, self-adjoint operator on $\mathcal{F}$. The functions (form factors) $G_{x_{e}}^{e}$ and $G_{x_{n}}^{n}$ on the right side of (2) are given by

$$
G_{x_{e}}^{e}(k)=e^{-i k \cdot x_{e}} \kappa_{e}(k), \quad G_{x_{n}}^{n}(k)=e^{-i k \cdot x_{n}} \kappa_{n}(k),
$$

where $\kappa_{e}$ and $\kappa_{n}$ belong to the Schwartz space, and

$$
\kappa_{e}(k)=\kappa_{n}(k)=0, \quad \text { for all } k \in \mathbb{R}^{3} \text { with }|k| \leq \sigma,
$$

for some $\sigma>0$ (infrared cutoff). In many of our results, we could pass to the limit $\sigma=0$; but in our proof of asymptotic completeness of Rayleigh scattering, the condition that $\sigma>0$ is essential. Finally, the parameter $g$ on r.h.s. of (2) is a coupling constant; it is assumed to be non-negative and will be chosen sufficiently small in the proofs of our results. (It should be noted that we are using units such that Planck's constant $\hbar=1$ and the speed of light $c=1$, and we work with dimensionless variables $x_{e}, x_{n}, k$ chosen such that $H_{\text {atom }}$ and $H_{f}$ are independent of $g$.)

In the description of the atom, it is natural to use the following variables:

$$
X=\frac{m_{e} x_{e}+m_{n} x_{n}}{m_{e}+m_{n}}, \quad x=x_{e}-x_{n} .
$$

Here $X$ is the position of the center of mass of the atom, and $x$ is the position of the electron relative to the one of the nucleus. Then

$$
H_{g}=\frac{P^{2}}{2 M}+\frac{p^{2}}{2 m}+V(x)+H_{f}+g\left(\phi\left(G_{X+\frac{m_{n}}{M} x}^{e}\right)+\phi\left(G_{X-\frac{m_{e}}{M} x}^{n}\right)\right),
$$

where $P=-i \nabla_{X}, p=-i \nabla_{x}, M=m_{e}+m_{n}$, and $m=m_{e} m_{n} M^{-1}$; (center-of-mass momentum, relative momentum, total mass, reduced mass, respectively). Self-adjointness of $H_{g}$ on $\mathcal{H}$ (under appropriate assumptions on $V$ ) is a standard result.

In this paper, we study the dynamics generated by $H_{g}$ on the subspace of states in $\mathcal{H}$ whose maximal energy is below the ionization threshold

$$
\Sigma_{\text {ion }}=\lim _{R \rightarrow \infty} \inf _{\varphi \in \mathcal{D}_{R}} \frac{\left\langle\varphi, H_{g} \varphi\right\rangle}{\langle\varphi, \varphi\rangle},
$$

where $\mathcal{D}_{R}=\{\varphi \in \mathcal{H}: \chi(|x| \geq R) \varphi=\varphi\}$ is the subspace of vectors in $\mathcal{H}$ with the property that the distance between the electron and the nucleus is at least $R$. Vectors in $\mathcal{H}$ with a maximal total energy below $\Sigma_{\text {ion }}$ exhibit exponential decay in $|x|$, the distance between the electron and the nucleus, see [Gr04]. Under our assumptions, $-C g^{2} \leq \Sigma_{\text {ion }} \leq 0$, for a finite constant $C$ depending only on $\kappa_{e}$ and $\kappa_{n}$. When $g \downarrow 0$ then $\Sigma_{\text {ion }} \uparrow 0$, which is the ionization threshold of a one-electron atom or -ion decoupled from the radiation field.

Our choice of the Hamiltonian $H_{g}$, see (2) and (5), and of the form factors $G_{x_{e}}^{e}$ and $G_{x_{n}}^{n}$, see (4), makes it clear that the dynamics of the system is space-translation invariant: Let

$$
P_{f}=\int \mathrm{d} k k a^{*}(k) a(k)
$$


denote the momentum operator of the radiation field, and let $\Pi=P+P_{f}$ be the total momentum operator. It is easy to check that

$$
\left[H_{g}, \Pi\right]=0 \quad \text { (translation invariance). }
$$

It is then useful to consider direct-integral decompositions of the space $\mathcal{H}$ and the operator $H_{g}$ over the spectrum of the total momentum operator $\Pi$ (which, as a set, is $\mathbb{R}^{3}$ ). Thus

$$
\mathcal{H}=\int_{\mathbb{R}^{3}}^{\oplus} \mathrm{d} \Pi \mathcal{H}_{\Pi}, \quad \text { with } \quad \mathcal{H}_{\Pi} \simeq L^{2}\left(\mathbb{R}^{3}, d x\right) \otimes \mathcal{F}
$$

and

$$
H_{g}=\int_{\mathbb{R}^{3}}^{\oplus} \mathrm{d} \Pi H_{g}(\Pi),
$$

where the fiber Hamiltonian $H_{g}(\Pi)$ is the operator on the fiber Hilbert space $\mathcal{H}_{\Pi}$ given by

$$
H_{g}(\Pi)=\frac{\left(\Pi-P_{f}\right)^{2}}{2 M}+H_{\mathrm{at}}+H_{f}+g\left(\phi\left(G_{\frac{m_{n}}{M} x}^{e}\right)+\phi\left(G_{-\frac{m_{e}}{M} x}^{n}\right)\right),
$$

where $\Pi-P_{f}$ is the center-of-mass momentum of the atom, and $H_{\text {at }}=p^{2} / 2 m+V(x)$ is the Hamiltonian describing the relative motion of the electron around the nucleus.

We are now in the position to summarize the main results proven in this paper for the model introduced above. In a first part, we analyze the energy spectra of the fiber Hamiltonians $H_{g}(\Pi)$ below a certain threshold $\Sigma<\min \left(\Sigma_{\text {ion }}, \Sigma_{\beta}\right)$, where $\Sigma_{\text {ion }}$ is given in (6), and $\Sigma_{\beta}=E_{0}^{\text {at }}+M \beta^{2} / 2 ; E_{0}^{\text {at }}$ is the ground state energy of $H_{\text {at }}$, and $\beta$ is a constant $<1$ (=speed of light, in our units). The condition $\Sigma<\Sigma_{\text {ion }}$ guarantees that the electron is bound to the nucleus, with exponential decay in $x$, and $\Sigma<\Sigma_{\beta<1}$ implies that, for a sufficiently small coupling constant $g$, the center-of-mass velocity of the atom is smaller than the speed of light. (For center-of-mass velocities $>1$, the ground state energy of the atom decoupled from the radiation field is embedded in continuous spectrum, and the ground state becomes unstable when the coupling to the radiation field is turned on.) For realistic atoms, in particular for hydrogen, $\Sigma_{\text {ion }} \ll \Sigma_{\beta=1 / 3}$, so that $\Sigma<\Sigma_{\text {ion }}$ is the only relevant condition. We let $E_{g}(\Pi)=\inf \sigma\left(H_{g}(\Pi)\right)$ denote the ground state energy of $H_{g}(\Pi)$, and we define

$$
B_{\Sigma}=\left\{\Pi \in \mathbb{R}^{3}: E_{0}^{\mathrm{at}}+\frac{\Pi^{2}}{2 M} \leq \Sigma\right\} .
$$

We prove that, for every $\Pi \in B_{\Sigma}, E_{g}(\Pi)$ is a simple eigenvalue of $H_{g}(\Pi)$, i.e., that the atom has a unique ground state, provided $g$ is small enough. This is a result that is expected to survive the limit $\sigma \downarrow 0$, provided the factors $\kappa_{e}$ and $\kappa_{n}$ are not too singular at $k=0$. For the Pauli-Fierz model of non-relativistic QED, existence of a ground state can be proven under conditions similar to the ones described above, provided the total charge of electrons and nucleus vanishes; see [AGG05].

By appropriately modifying Mourre theory in a form developed in [BFSS99], we prove that the spectrum of $H_{g}(\Pi)$ in the interval $\left(E_{g}(\Pi), \Sigma\right)$ is purely continuous. With relatively little further effort, our methods would also show that $\sigma\left(H_{g}(\Pi)\right) \cap\left(E_{g}(\Pi), \Sigma\right)$ is absolutely continuous. (These results, too, would survive the removal of the infrared cutoff, $\sigma \downarrow 0$. This will not be shown in this paper; but see [FGSi05].) 
We denote the ground state of $H_{g}(\Pi), \Pi \in B_{\Sigma}$, by $\psi_{\Pi} ;\left(\psi_{\Pi}\right.$ is called the dressed atom (ground) state of momentum $\Pi$ ). The space of wave packets of dressed atom states, $\mathcal{H}_{\text {das }}$, is the subspace of the total Hilbert space $\mathcal{H}$ given by

$$
\mathcal{H}_{\text {das }}=\left\{\psi(f): \psi(f)=\int^{\oplus} \mathrm{d} \Pi f(\Pi) \psi_{\Pi}, f \in L^{2}\left(B_{\Sigma}, \mathrm{d} \Pi\right)\right\} .
$$

This space is invariant under the time evolution. In fact, $e^{-i H_{g} t} \psi(f)=\psi\left(f_{t}\right)$, where, for $f \in L^{2}\left(B_{\Sigma}, \mathrm{d} \Pi\right), f_{t}(\Pi)=e^{-i E_{g}(\Pi) t} f(\Pi) \in L^{2}\left(B_{\Sigma}, \mathrm{d} \Pi\right)$, for all times $t$.

In a second part of our paper, scattering theory is developed for the models introduced above. We first construct asymptotic photon creation- and annihilation operators

$$
a_{ \pm}^{\sharp}(h) \varphi=s-\lim _{t \rightarrow \pm \infty} e^{i H_{g} t} a^{\sharp}\left(h_{t}\right) e^{-i H_{g} t} \varphi,
$$

where $h_{t}(k)=e^{-i|k| t} h(k)$ is the free time evolution of a one-photon state $h(k)$. To ensure the existence of the strong limit on the r.h.s. of (14), we assume that $h \in L^{2}\left(\mathbb{R}^{3},(1+\right.$ $\left.\left.|k|^{-1}\right) \mathrm{d} k\right)$, that $\varphi$ belongs to the range of the spectral projection, $E_{\Sigma}\left(H_{g}\right)$, of $H_{g}$ corresponding to the interval $\left(-\infty, \Sigma\right.$ ], with $\Sigma<\left(\Sigma_{\text {ion }}, \Sigma_{\beta}\right)$, as above, and $\beta<1$, and that the coupling constant $g$ is so small (depending on $\Sigma$ ) that the velocity of the center of mass of the atom is smaller than one. The last condition ensures that the distance between the atom and a configuration of outgoing photons increases to infinity and hence the interaction between these photons and the atom tends to 0 , as time $t$ tends to $+\infty$. The details of the proof of (14) are very similar to those in [FGS00]. From (13) and (14) we infer that, for $\psi(f) \in \mathcal{H}_{\text {das }}$, and under the conditions of existence of the limit in (14), vectors of the form $a_{ \pm}^{*}\left(h_{1}\right) \cdots a_{ \pm}^{*}\left(h_{n}\right) \psi(f)$ exist, and their time evolution is the one of freely moving photons and a freely moving atom:

$$
e^{-i H_{g} t} a_{ \pm}^{*}\left(h_{1}\right) \ldots a_{ \pm}^{*}\left(h_{n}\right) \psi(f)=a^{*}\left(h_{1, t}\right) \cdots a^{*}\left(h_{n, t}\right) \psi\left(f_{t}\right)+o(1)
$$

as $t \rightarrow \pm \infty$. Furthermore, $a_{ \pm}(h) \psi(f)=0$, under the same assumptions. Equation (15) provides the justification for calling the vectors $a_{ \pm}^{*}\left(h_{1}\right) \ldots a_{ \pm}^{*}\left(h_{n}\right) \psi(f)$ scattering states. We already know that the atom does not have any stable excited states. It is therefore natural to expect that the time evolution of an arbitrary vector in the range of the spectral projection $E_{\Sigma}\left(H_{g}\right)$, with $\Sigma<\min \left(\Sigma_{\text {ion }}, \Sigma_{\beta<1}\right)$ as above, approaches a vector describing a configuration of freely moving photons and a freely moving atom in its ground state, as time $t$ tends to $\pm \infty$. Thus, with (15) and (13), we expect that, for $\Sigma<\min \left(\Sigma_{\text {ion }}, \Sigma_{\beta<1}\right)$,

$$
\begin{gathered}
\left\langle\left\{ a_{ \pm}^{*}\left(h_{1}\right) \ldots a_{ \pm}^{*}\left(h_{n}\right) \psi(f): \psi(f) \in \mathcal{H}_{\mathrm{das}}, h_{j} \in L^{2}\left(\mathbb{R}^{3},(1+1 /|k|) \mathrm{d} k\right),\right.\right. \\
j=1, \ldots, n, n=1,2, \ldots\}\rangle^{-} \supset \operatorname{Ran} E_{\Sigma}\left(H_{g}\right)
\end{gathered}
$$

where $\langle S\rangle$ denotes the linear subspace spanned by a set, $S$, of vectors in $\mathcal{H}$, and $\langle S\rangle^{-}$ denotes the closure of $\langle S\rangle$ in the norm of $\mathcal{H}$. Property (16) is called asymptotic completeness of Rayleigh scattering. The main result of this paper is a proof of (16) under the supplementary condition that $\Sigma<\Sigma_{\beta}$ for some $\beta<1 / 3$ (the proof of (16) is the only part of the paper where, for technical reasons, we need to assume $\beta<1 / 3$; all other results only require $\beta<1$ ). Next, we reformulate (16) in a more convenient language. 
We define a Hilbert space of scattering states as the space $\mathcal{H}_{\text {das }} \otimes \mathcal{F}$, and we introduce an asymptotic Hamilton operator, $\tilde{H}_{g}^{\text {das }}$, by setting

$$
\tilde{H}_{g}^{\text {das }}=H_{g}^{\text {das }} \otimes 1+1 \otimes H_{f},
$$

where

$$
H_{g}^{\text {das }} \psi(f)=\psi\left(E_{g}(.) f\right)
$$

for arbitrary $f \in L^{2}\left(B_{\Sigma}, \mathrm{d} \Pi\right)$, with $\Sigma<\min \left(\Sigma_{\text {ion }}, \Sigma_{\beta<1 / 3}\right)$, as above. On the range of $E_{\Sigma}\left(\tilde{H}_{g}^{\text {das }}\right)$, the operators $\Omega_{ \pm}$, given by

$$
\Omega_{ \pm}\left(\psi(f) \otimes a^{*}\left(h_{1}\right) \ldots a^{*}\left(h_{n}\right) \Omega\right)=a_{ \pm}^{*}\left(h_{1}\right) \ldots a_{ \pm}^{*}\left(h_{n}\right) \psi(f)
$$

exist; see (14). The vector $\Omega$ is the vacuum in the Fock-space characterized by the property that $a(h) \Omega=0$, for $h \in L^{2}\left(\mathbb{R}^{3}, \mathrm{~d} k\right)$. The operators $\Omega_{+}$and $\Omega_{-}$are called wave operators, and the scattering matrix is defined by

$$
S=\Omega_{+}^{*} \Omega_{-} .
$$

From Eqs. (14) and (15) we find that

$$
e^{-i H_{g} t} \Omega_{ \pm}=\Omega_{ \pm} e^{-i \tilde{H}_{g}^{\mathrm{das}} t}
$$

and hence the ranges of $\Omega_{+}$and $\Omega_{-}$are contained in the range of $E_{\Sigma}\left(H_{g}\right)$. Using that $a_{ \pm}(h) \psi(f)=0$, for $h \in L^{2}\left(\mathbb{R}^{3}, \mathrm{~d} k\right)$ and $\psi(f) \in \mathcal{H}_{\text {das }}$, one sees that $\Omega_{+}$and $\Omega_{-}$are isometries from the range of $E_{\Sigma}\left(\tilde{H}_{g}^{\text {das }}\right)$ into $\mathcal{H}$. If we succeeded in proving that

$$
\operatorname{Ran}\left(\Omega_{ \pm}\left\lceil\operatorname{Ran} E_{\Sigma}\left(\tilde{H}_{g}^{\mathrm{das}}\right)\right)=\operatorname{Ran} E_{\Sigma}\left(H_{g}\right)\right.
$$

we would have established the unitarity of the $S$-matrix, defined in (20), on $\operatorname{Ran} E_{\Sigma}\left(\tilde{H}^{\text {das }}\right)$, i.e., asymptotic completeness of Rayleigh scattering.

In order to prove (21), we show that $\Omega_{ \pm}$have right inverses defined on $\operatorname{Ran} E_{\Sigma}\left(H_{g}\right)$. Our proof is inspired by proofs of similar results in [DG99] and in [FGS02, FGS04]. It is based on constructing a so-called asymptotic observable $W$ and then proving that $W$ is positive on the orthogonal complement of $\mathcal{H}_{\text {das }}$ in $\operatorname{Ran} E_{\Sigma}\left(H_{g}\right)$. The proof of this last result is, perhaps, the most original accomplishment in this paper and is based on some new ideas.

Our paper is organized as follows. In Sect. 2, we define our model more precisely, and we state our assumptions on the potential $V(x)$ and on the form factors $G_{x_{e}}^{e}$ and $G_{x_{n}}^{n}$. In Sect. 3, we study the spectrum of the fiber Hamiltonian $H_{g}(\Pi)$ : In Sect. 3.1, we prove the existence of dressed atom states, and, in Sect. 3.3, we prove two positive commutator estimates, from which we conclude that the spectrum of $H_{g}(\Pi)$ above the ground state energy and below an appropriate threshold is continuous. In Sect. 4, we discuss the scattering theory of the system. First, in Sect. 4.1, we prove the existence of asymptotic field operators, we recall some of their properties, we prove the existence of the wave operators, and we state our main theorem. Then, in Sect. 4.2, we introduce a modified Hamiltonian, $H_{\text {mod }}$, describing "massive" photons, and we explain why it is enough to prove asymptotic completeness for $H_{\text {mod }}$ instead of $H_{g}$. In Sect. 4.3, we construct asymptotic observables $W$ and inverse wave operators $W_{ \pm}$. In Sect. 4.4, we 
prove positivity of our asymptotic observables when restricted to the orthogonal complement of $\mathcal{H}_{\text {das }}$ (the space of wave packets of dressed atom states). Finally, in Sect. 4.5, we complete the proof of asymptotic completeness. In Appendix A, we introduce some notation, used throughout the paper, concerning operators on the bosonic Fock space. In Appendix B, we summarize bounds used to control the interaction between the electron (or the nucleus) and the radiation field.

\section{The Model}

We consider a non-relativistic atom consisting of a nucleus and an electron interacting through a two-body potential $V(x)$. The Hamiltonian describing the dynamics of the atom is the self-adjoint operator

$$
H_{\text {atom }}=\frac{p_{n}^{2}}{2 m_{n}}+\frac{p_{e}^{2}}{2 m_{e}}+V\left(x_{n}-x_{e}\right)
$$

acting on the Hilbert space $\mathcal{H}_{\text {atom }}=L^{2}\left(\mathbb{R}^{3}, \mathrm{~d} x_{n}\right) \otimes L^{2}\left(\mathbb{R}^{3}, \mathrm{~d} x_{e}\right)$, where $x_{n}$ and $x_{e}$ denote the position of the nucleus and of the electron, respectively, and $p_{n}=-i \nabla_{x_{n}}$ and $p_{e}=-i \nabla_{x_{e}}$ are the corresponding momenta. We assume that the interaction potential $V(x)$ satisfies the following assumptions.

Hypothesis (H0). The potential $V$ is a real-valued, locally square integrable function on $\mathbb{R}^{3}$ with

$$
\lim _{|x| \rightarrow \infty} V(x)=0
$$

\section{Remarks.}

1) Hypothesis (H0) is satisfied by the Coulomb potential $V(x)=-1 /|x|$ and it is inspired by this potential. It guarantees that (a), $V \in L^{2}\left(\mathbb{R}^{3}\right)+L^{\infty}\left(\mathbb{R}^{3}\right)$, and that (b), $V \in R+L_{\varepsilon}^{\infty}\left(\mathbb{R}^{3}\right)$, where $R$ denotes the Rollnik class. Note that $L_{\text {loc }}^{2} \subset L_{\text {loc }}^{3 / 2}$ and that $L^{3 / 2}\left(\mathbb{R}^{3}\right) \subset R$. By (a), $V$ is infinitesimally small w.r.to $p^{2}=-\Delta$, and hence $p^{2} / 2 m+V$, for any $m>0$, is self-adjoint on $D\left(p^{2}\right)=H^{2}\left(\mathbb{R}^{3}\right)$ and bounded from below. If inf $\sigma\left(p^{2} / 2 m+V\right)$ is an eigenvalue, then, by (b), it is non-degenerate [RS78, Theorem XIII.46].

Similarly the Hamiltonian $H_{\text {atom }}$ with domain $H^{2}\left(\mathbb{R}_{x_{n}}^{3} \times \mathbb{R}_{x_{e}}^{3}\right)$ is a self-adjoint operator on the Hilbert space $L^{2}\left(\mathbb{R}^{3}, \mathrm{~d} x_{n}\right) \otimes L^{2}\left(\mathbb{R}^{3}, \mathrm{~d} x_{e}\right)$ and it is bounded from below.

2) With little more effort we could have covered a much larger class of locally square integrable potentials $V$ where only the negative part $V_{-}(x)=\max \{-V(x), 0\}$ is infinitesimally small with respect to $\Delta$ and $H_{\text {atom }}$ is self-adjointly realized in terms of a Friedrich's extension. This would allow, e.g., for confining potentials that tend to $\infty$ as $|x| \rightarrow \infty$.

3) Note that we are neglecting the degrees of freedom corresponding to the spin of the nucleus and of the electron, because they do not play an interesting role in the scattering process.

Next we couple the atom to a quantized scalar radiation field. We call the particles described by the quantized field (scalar) photons. The pure states of the photon field are vectors in the bosonic Fock space over the one-particle space $L^{2}\left(\mathbb{R}^{3}, \mathrm{~d} k\right)$,

$$
\mathcal{F}=\bigoplus_{n \geq 0} L_{s}^{2}\left(\mathbb{R}^{3 n}, \mathrm{~d} k_{1} \ldots \mathrm{d} k_{n}\right),
$$


where $L_{s}^{2}\left(\mathbb{R}^{3 n}\right)$ denotes the subspace of $L^{2}\left(\mathbb{R}^{3 n}\right)$ consisting of all functions which are completely symmetric under permutations of the $n$ arguments. The variables $k_{1}, \ldots k_{n}$ denote the momenta of the photons.

The dispersion relation of the photons is given by $\omega(k)=|k|$, which characterizes relativistic particles with zero mass. The free Hamiltonian of the quantized radiation field is given by the second quantization of $\omega(k)=|k|$, denoted by $\mathrm{d} \Gamma(|k|)$. Formally,

$$
\mathrm{d} \Gamma(|k|)=\int \mathrm{d} k|k| a^{*}(k) a(k),
$$

where $a^{*}(k)$ and $a(k)$ are the usual creation- and annihilation operators on $\mathcal{F}$, satisfying the canonical commutation relations $\left[a^{\sharp}(k), a^{\sharp}\left(k^{\prime}\right)\right]=0,\left[a(k), a^{*}\left(k^{\prime}\right)\right]=\delta\left(k-k^{\prime}\right)$. More notations for operators on Fock space that are used throughout the paper are collected in Appendix A.

The total system, atom plus quantized radiation field, has the Hilbert space $\mathcal{H}=$ $\mathcal{H}_{\text {atom }} \otimes \mathcal{F}$; its dynamics is generated by the Hamiltonian

$$
H_{g}=H_{\text {atom }}+\mathrm{d} \Gamma(|k|)+g\left(\phi\left(G_{x_{e}}^{e}\right)+\phi\left(G_{x_{n}}^{n}\right)\right),
$$

where $g$ is a real non-negative coupling constant (the assumption $g \geq 0$ is not needed, it just makes the notation a little bit simpler), and where

$$
\phi\left(G_{x}\right)=\int \mathrm{d} k\left(a^{*}(k) G_{x}(k)+a(k) \bar{G}_{x}(k)\right) .
$$

The form factors $G_{x}^{e}$ and $G_{x}^{n}$ are square integrable functions of $k$ with values in the multiplication operators on $L^{2}\left(\mathbb{R}^{3}, \mathrm{~d} x\right)$. Clearly, $G_{x}^{e}$ describes the interaction between the electron and the radiation field, and $G_{x}^{n}$ couples the field to the nucleus. The next hypothesis specifies our assumptions on the form factors $G_{x}^{e}$ and $G_{x}^{n}$.

Hypothesis (H1). The form factors $G_{x}^{e}$ and $G_{x}^{n}$ have the form

$$
G_{x}^{e}(k)=e^{-i k \cdot x} \kappa_{e}(k) \text { and } G_{x}^{n}(k)=e^{-i k \cdot x} \kappa_{n}(k),
$$

where $\kappa_{e}, \kappa_{n}$ belong to Schwartz space $\mathcal{S}\left(\mathbb{R}^{3}\right)$, and $\kappa_{e}(k)=\kappa_{n}(k)=0$ if $|k| \leq \sigma$, for some $\sigma>0$.

The particular form of $G_{x}^{e}$ and $G_{x}^{n}$ given in (27) guarantees the translation invariance of the system (see the discussion after (37)). The presence of an infrared cutoff $\sigma>0$ in $\kappa_{e}$ and $\kappa_{n}$ is used in the proofs of many of our results; but it is not necessary for the existence of the asymptotic field operators and for the existence of the wave operator in Sect. 4.1. Notice that, even though our main results require the coupling constant $g$ to be sufficiently small, how small $g$ has to be does not depend on the infrared cutoff $\sigma$, in the following sense. If we define $\kappa_{e}(k)=\tilde{\kappa}_{e}(k) \chi(|k| / \sigma)$ and $\kappa_{n}(k)=\tilde{\kappa}_{n}(k) \chi(|k| / \sigma)$, with $\tilde{\kappa}_{e, n} \in C_{0}^{\infty}\left(\mathbb{R}^{3}\right)$, and with $\chi \in C^{\infty}(\mathbb{R})$ monotone increasing and such that $\chi(s)=0$ if $s \leq 1$ and $\chi(s)=1$ if $s \geq 2$, then Hypothesis (H1) is satisfied for every choice of $\sigma>0$. Moreover how small $g$ has to be is independent of the choice of the parameter $\sigma$.

Assuming Hypotheses (H0) and (H1), the Hamiltonian $H_{g}$, defined on the domain $H^{2}\left(\mathbb{R}_{x_{e}}^{3} \times \mathbb{R}_{x_{n}}^{3}\right) \otimes D(\mathrm{~d} \Gamma(|k|))$, is essentially self-adjoint and bounded from below. This follows from Lemma 20, which shows, using Hypothesis (H1), that the interaction $\phi\left(G_{x_{e}}^{e}\right)+\phi\left(G_{x_{n}}^{n}\right)$ is infinitesimal with respect to the free Hamiltonian $H_{0}=H_{\text {atom }}+$ $\mathrm{d} \Gamma(|k|)$. 
To study the system described by the Hamiltonian $H_{g}$, it is more convenient to use coordinates describing the center of mass of the atom and the relative position of the nucleus and the electron. We define

$$
X=\frac{m_{n} x_{n}+m_{e} x_{e}}{m_{n}+m_{e}}, \quad x=x_{e}-x_{n} .
$$

Then, the atomic Hamiltonian $H_{\text {atom }}$ becomes

$$
H_{\text {atom }}=\frac{P^{2}}{2 M}+\frac{p^{2}}{2 m}+V(x),
$$

where $P=-i \nabla_{X}$ is the center of mass momentum of the atom, and $p=-i \nabla_{x}$ is the momentum conjugate to the relative coordinate $x$. Moreover, $M=m_{e}+m_{n}$ is the total atomic mass, and $m=\left(m_{e}^{-1}+m_{n}^{-1}\right)^{-1}$ is the reduced mass. Expressed in the new coordinates, the total Hamiltonian of the system is given by

$$
\begin{aligned}
H_{g} & =\frac{P^{2}}{2 M}+\frac{p^{2}}{2 m}+V(x)+\mathrm{d} \Gamma(|k|)+g\left(\phi\left(G_{X+\lambda_{e} x}^{e}\right)+\phi\left(G_{X-\lambda_{n} x}^{n}\right)\right) \\
& =\frac{P^{2}}{2 M}+\frac{p^{2}}{2 m}+V(x)+\mathrm{d} \Gamma(|k|)+g \phi\left(G_{X, x}\right) .
\end{aligned}
$$

Here $\lambda_{e}=m_{n} / M$ and $\lambda_{n}=m_{e} / M$, and we use the notation

$$
G_{X, x}(k)=G_{X+\lambda_{e} x}^{e}(k)+G_{X-\lambda_{n} x}^{n}(k)=e^{-i k \cdot X} F_{x}(k),
$$

with

$$
F_{x}(k)=e^{-i \lambda_{e} k \cdot x} \kappa_{e}(k)+e^{i \lambda_{n} k \cdot x} \kappa_{n}(k) .
$$

The fact that the form factors $\kappa_{e}$ and $\kappa_{n}$ contain an infrared cutoff (meaning that $\kappa_{n}(k)=$ $\kappa_{e}(k)=0$, if $\left.|k| \leq \sigma\right)$ implies that photons with very small momenta do not interact with the atom. In other words, they decouple from the rest of the system. We denote by $\chi_{i}$ (the subscript $i$ stands for "interacting") the characteristic function of the set $\left\{k \in \mathbb{R}^{3}:|k| \geq \sigma\right\}$. Then the operator $\Gamma\left(\chi_{i}\right)$, whose action on the $n$-particle sector of $\mathcal{F}$ is given by

$$
\Gamma\left(\chi_{i}\right)=\chi_{i} \otimes \chi_{i} \otimes \cdots \otimes \chi_{i}
$$

defines the orthogonal projection onto states without soft bosons. The fact that soft bosons do not interact with the atom implies that $H_{g}$ leaves the range of $\Gamma\left(\chi_{i}\right)$ invariant; $H_{g}$ commutes with $\Gamma\left(\chi_{i}\right)$. Another way to isolate the soft, non-interacting, photons from the rest of the system is as follows. We have that $L^{2}\left(\mathbb{R}^{3}\right)=L^{2}\left(B_{\sigma}(0)\right) \oplus L^{2}\left(B_{\sigma}(0)^{c}\right)$, where $B_{\sigma}(0)$ is the open ball of radius $\sigma$ around the origin and $B_{\sigma}(0)^{c}$ denotes its complement. Hence the Fock space can be decomposed as $\mathcal{F} \simeq \mathcal{F}_{i} \otimes \mathcal{F}_{s}$, where $\mathcal{F}_{i}$ is the bosonic Fock space over $L^{2}\left(B_{\sigma}(0)^{c}\right)$ (describing interacting photons), and $\mathcal{F}_{s}$ is the bosonic Fock space over $L^{2}\left(B_{\sigma}(0)\right)$ (describing soft, non-interacting, photons). Accordingly, the Hilbert space $\mathcal{H}=L^{2}\left(\mathbb{R}^{3}, \mathrm{~d} X\right) \otimes L^{2}\left(\mathbb{R}^{3}, \mathrm{~d} x\right) \otimes \mathcal{F}$ can be decomposed as

$$
\begin{aligned}
\mathcal{H} & \simeq \mathcal{H}_{i} \otimes \mathcal{F}_{s} \quad \text { with } \\
\mathcal{H}_{i} & =L^{2}\left(\mathbb{R}^{3}, \mathrm{~d} X\right) \otimes L^{2}\left(\mathbb{R}^{3}, \mathrm{~d} x\right) \otimes \mathcal{F}_{i} .
\end{aligned}
$$


By $U: \mathcal{H} \rightarrow \mathcal{H}_{i} \otimes \mathcal{F}_{s}$ we denote the unitary map from $\mathcal{H}$ to $\mathcal{H}_{i} \otimes \mathcal{F}_{s}$. The action of the Hamiltonian $H_{g}$ on $\mathcal{H}_{i} \otimes \mathcal{F}_{s}$ is then given by

$$
U H_{g} U^{*}=H_{i} \otimes 1+1 \otimes \mathrm{d} \Gamma(|k|)
$$

with

$$
H_{i}=H_{g}\left\lceil\mathcal{H}_{i}\right.
$$

Note that in the representation of the system on the Hilbert space $\mathcal{H}_{i} \otimes \mathcal{F}_{s}$, the projection $\Gamma\left(\chi_{i}\right)$ (projecting on states without soft bosons) is simply given by $U \Gamma\left(\chi_{i}\right) U^{*}=1 \otimes P_{\Omega}$, where $P_{\Omega}$ denotes the orthogonal projection onto the vacuum $\Omega$ in $\mathcal{F}_{s}$.

One of the most important properties of the Hamiltonian $H_{g}$ is its invariance with respect to translations of the whole system, atom and field. More precisely, defining the total momentum of the system by

$$
\Pi=P+\mathrm{d} \Gamma(k),
$$

we have that $\left[H_{g}, \Pi\right]=0$. Because of this property, it can be useful to rewrite the Hilbert space $\mathcal{H}=L^{2}\left(\mathbb{R}^{3}, \mathrm{~d} X\right) \otimes L^{2}\left(\mathbb{R}^{3}, \mathrm{~d} x\right) \otimes \mathcal{F}$ as a direct integral over fibers with fixed total momentum. Specifically, we define the isomorphism

$$
T: L^{2}\left(\mathbb{R}^{3}, \mathrm{~d} X\right) \otimes L^{2}\left(\mathbb{R}^{3}, \mathrm{~d} x\right) \otimes \mathcal{F} \longrightarrow L^{2}\left(\mathbb{R}^{3}, \mathrm{~d} \Pi ; L^{2}\left(\mathbb{R}^{3}, \mathrm{~d} x\right) \otimes \mathcal{F}\right)
$$

as follows. For $\psi=\left\{\psi^{(n)}\left(X, x, k_{1}, \ldots, k_{n}\right)\right\}_{n \geq 0} \in L^{2}\left(\mathbb{R}^{3}, \mathrm{~d} X\right) \otimes L^{2}\left(\mathbb{R}^{3}, \mathrm{~d} x\right) \otimes \mathcal{F}$, we define

$$
(T \psi)(\Pi)=\left\{(T \psi)_{\Pi}^{(n)}\left(x, k_{1}, \ldots k_{n}\right)\right\}_{n \geq 0} \in L^{2}\left(\mathbb{R}^{3}, \mathrm{~d} x\right) \otimes \mathcal{F}
$$

with

$$
(T \psi)_{\Pi}^{(n)}\left(x, k_{1}, \ldots k_{n}\right)=\widehat{\psi^{(n)}}\left(\Pi-k_{1}-\cdots-k_{n}, x, k_{1}, \ldots k_{n}\right),
$$

where

$$
\widehat{\psi^{(n)}}\left(P, x, k_{1}, \ldots, k_{n}\right)=\frac{1}{(2 \pi)^{3 / 2}} \int \mathrm{d} X e^{-i P \cdot X} \psi^{(n)}\left(X, x, k_{1}, \ldots, k_{n}\right)
$$

is the Fourier transform of $\psi^{(n)}$ with respect to its first variable. Because of its translation invariance, the Hamiltonian $H_{g}$ leaves invariant each fiber with fixed total momentum of the Hilbert space $L^{2}\left(\mathbb{R}^{3}, \mathrm{~d} \Pi ; L^{2}\left(\mathbb{R}^{3}, \mathrm{~d} x\right) \otimes \mathcal{F}\right) \simeq \int^{\oplus}\left(L^{2}\left(\mathbb{R}^{3}, \mathrm{~d} x\right) \otimes \mathcal{F}\right) \mathrm{d} \Pi$. More precisely,

$$
\begin{aligned}
\left(T^{*} H_{g} T \psi\right)(\Pi) & =H_{g}(\Pi) \psi(\Pi) \text { with } \\
H_{g}(\Pi) & =\frac{(\Pi-\mathrm{d} \Gamma(k))^{2}}{2 M}+\mathrm{d} \Gamma(|k|)+H_{\mathrm{at}}+g \phi\left(F_{x}\right),
\end{aligned}
$$

where we put $H_{\text {at }}=p^{2} / 2 m+V$. Recall that $F_{x}(k)=e^{-i \lambda_{e} k \cdot x} \kappa_{e}(k)+e^{i \lambda_{n} k \cdot x} \kappa_{n}(k)$.

Note that, for every fixed $\Pi$, the operator $H_{g}(\Pi)$ is a self-adjoint operator on the fiber space $L^{2}\left(\mathbb{R}^{3}, \mathrm{~d} x\right) \otimes \mathcal{F}$. Our first results, stated in the next section, describe the structure of the spectrum of the fiber-Hamiltonian $H_{g}(\Pi)$, for fixed values of $\Pi$. 


\section{The Spectrum of $H_{g}(\Pi)$}

3.1. Dressed atom states. The first question arising in the analysis of the spectrum of

$$
H_{g}(\Pi)=\frac{(\Pi-\mathrm{d} \Gamma(k))^{2}}{2 M}+\mathrm{d} \Gamma(|k|)+H_{\mathrm{at}}+g \phi\left(F_{x}\right)
$$

concerns the existence of a ground state of $H_{g}(\Pi)$ : we wish to know whether or not $E_{g}(\Pi)=\inf \sigma\left(H_{g}(\Pi)\right)$ is an eigenvalue of $H_{g}(\Pi)$. We will answer this question affirmatively, under the assumption that the energy $E_{g}(\Pi)$ lies below some threshold and that the coupling constant is sufficiently small. The restriction to small energies is necessary to guarantee that the center of mass of the atom does not move faster than with the speed of light ( $c=1$ in our units), and that the atom is not ionized. The following lemma (and its corollary) proves that an upper bound on the total energy is sufficient to bound the momentum of the center of mass of the atom (provided the coupling constant is sufficiently small) and to make sure that the electron is exponentially localized near the nucleus.

Lemma 1. Assume that Hypotheses (HO) and (HI) are satisfied.

i) Define $E_{0}^{a t}=\inf \sigma\left(H_{a t}\right)$, and fix $\beta>0$. Suppose $\Sigma<\Sigma_{\beta}=E_{0}^{a t}+(M / 2) \beta^{2}$. Then there is $g_{\Sigma, \beta}>0$ such that

$$
\left\|\frac{|\Pi-\mathrm{d} \Gamma(k)|}{M} E_{\Sigma}\left(H_{g}(\Pi)\right)\right\| \leq \beta
$$

for all $g \leq g_{\Sigma, \beta}(g \geq 0)$, and for all $\Pi \in \mathbb{R}^{3}$. In particular $\left\|(|P| / M) E_{\Sigma}\left(H_{g}\right)\right\| \leq \beta$. ii) Define the ionization threshold

$$
\Sigma_{i o n}=\lim _{R \rightarrow \infty} \inf _{\varphi \in \mathcal{D}_{R}}\left\langle\varphi, H_{g} \varphi\right\rangle
$$

with $\mathcal{D}_{R}=\left\{\varphi \in D\left(H_{g}\right): \chi(|x| \geq R) \varphi=\varphi\right\}$. Let $\Sigma, \alpha \in \mathbb{R}$ be such that $\Sigma+$ $\alpha^{2} /(2 m)<\Sigma_{i o n}$. Then

$$
\sup _{\Pi \in \mathbb{R}^{3}}\left\|e^{\alpha|x|} E_{\Sigma}\left(H_{g}(\Pi)\right)\right\|<\infty .
$$

Proof. i) Fix $\varepsilon>0$ such that $\Sigma+\varepsilon<\Sigma_{\beta}=E_{0}^{\text {at }}+(M / 2) \beta^{2}$. Choose $\chi \in C_{0}^{\infty}(\mathbb{R})$ such that $\chi(s)=1$ for $s \leq \Sigma$, and $\chi(s)=0$ if $s>\Sigma+\varepsilon$. Then we have that

$$
\begin{aligned}
\left\|(|\Pi-\mathrm{d} \Gamma(k)| / M) E_{\Sigma}\left(H_{g}(\Pi)\right)\right\| & \leq\left\|(|\Pi-\mathrm{d} \Gamma(k)| / M) \chi\left(H_{g}(\Pi)\right)\right\| \\
& \leq\left\|(|\Pi-\mathrm{d} \Gamma(k)| / M) \chi\left(H_{0}(\Pi)\right)\right\|+C g,
\end{aligned}
$$

where $H_{0}(\Pi)=(\Pi-\mathrm{d} \Gamma(k))^{2} / 2 M+H_{\mathrm{at}}+\mathrm{d} \Gamma(|k|)$ is the non-interacting fiber Hamiltonian and where the constant $C$ is independent of $\Pi$. To prove the last equation note that, if $\tilde{\chi}$ denotes an almost analytic extension of $\chi$ (see Appendix A in [FGS04] for a short introduction to the Helffer-Sjöstrand functional calculus), we have that

$$
\chi\left(H_{g}(\Pi)\right)-\chi\left(H_{0}(\Pi)\right)=\frac{1}{\pi} \int \mathrm{d} x \mathrm{~d} y \partial_{\bar{z}} \tilde{\chi}(z) \frac{1}{H_{0}(\Pi)-z} g \phi\left(F_{x}\right) \frac{1}{H_{g}(\Pi)-z},
$$


and therefore

$$
\begin{aligned}
& \left\||\Pi-\mathrm{d} \Gamma(k)|\left(\chi\left(H_{g}(\Pi)\right)-\chi\left(H_{0}(\Pi)\right)\right)\right\| \\
& \quad \leq C g\left\||\Pi-\mathrm{d} \Gamma(k)|\left(H_{0}(\Pi)+i\right)^{-1}\right\|\left\|\phi\left(F_{x}\right)\left(H_{g}(\Pi)+i\right)^{-1}\right\| \leq C g,
\end{aligned}
$$

uniformly in $\Pi$. Next, since $H_{\mathrm{at}} \geq E_{0}^{\mathrm{at}}=\inf \sigma\left(H_{\mathrm{at}}\right), \mathrm{d} \Gamma(|k|) \geq 0$, and by the definition of $\chi$, we have that

$$
\chi\left(H_{0}(\Pi)\right)=E_{\Sigma+\varepsilon-E_{0}^{\mathrm{at}}}\left(\frac{(\Pi-\mathrm{d} \Gamma(k))^{2}}{2 M}\right) \chi\left(H_{0}(\Pi)\right) .
$$

Since $\Sigma+\varepsilon-E_{0}^{\text {at }}<(1 / 2) M \beta^{2}$, we conclude from (46) that

$$
\left\|\frac{|\Pi-\mathrm{d} \Gamma(k)|}{M} E_{\Sigma}\left(H_{g}(\Pi)\right)\right\| \leq \beta
$$

for $g$ sufficiently small (independently of П).

As for part ii), we use Theorem 1 of [Gr04] and an estimate from its proof. Given $R \geq 0$ and $\Pi \in \mathbb{R}^{3}$, let

$$
\begin{aligned}
\Sigma_{R} & =\inf _{\varphi \in \mathcal{D}_{R},\|\varphi\|=1}\left\langle\varphi, H_{g} \varphi\right\rangle, \\
\Sigma_{R}(\Pi) & =\inf _{\varphi \in \mathcal{D}_{R, \Pi},\|\varphi\|=1}\left\langle\varphi, H_{g}(\Pi) \varphi\right\rangle,
\end{aligned}
$$

where $\mathcal{D}_{R}=\left\{\varphi \in D\left(H_{g}\right): \chi(|x| \leq R) \varphi=0\right\}$ and $\mathcal{D}_{R, \Pi}=\left\{\varphi \in D\left(H_{g}(\Pi)\right): \chi(|x| \leq\right.$ $R) \varphi=0\}$. Suppose for a moment that

$$
\Sigma_{R}(\Pi) \geq \Sigma_{R}
$$

for all $\Pi \in \mathbb{R}^{3}$ and all $R \in \mathbb{R}$. Then $\lim _{R \rightarrow \infty} \Sigma_{R}(\Pi) \geq \Sigma_{\text {ion }}$ and hence

$$
\left\|e^{\alpha|x|} E_{\Sigma}\left(H_{g}(\Pi)\right)\right\|<\infty
$$

by [Gr04, Theorem 1]. Moreover, the value of the parameter $R$ in the proof of [Gr04, Theorem 1], in the case of the Hamiltonian $H_{g}(\Pi)$, can be chosen independent of $\Pi$ thanks to (49). It follows that the estimate for $\left\|e^{\alpha|x|} E_{\Sigma}\left(H_{g}(\Pi)\right)\right\|$ from that proof is also independent of $\Pi$. It thus remains to prove (49). To this end we proceed by contradiction, assuming that (49) is wrong. Then there exist $\Pi_{0} \in \mathbb{R}^{3}$ and $\varepsilon>0$ such that

$$
\Sigma_{R}\left(\Pi_{0}\right)=\Sigma_{R}-\varepsilon .
$$

Hence, we find $\varphi_{0} \in \mathcal{D}_{R, \Pi_{0}} \subset L^{2}\left(\mathbb{R}^{3}, \mathrm{~d} x\right) \otimes \mathcal{F}$ with $\left\|\varphi_{0}\right\|=1$ and with

$$
\left\langle\varphi_{0}, H_{g}\left(\Pi_{0}\right) \varphi_{0}\right\rangle \leq \Sigma_{R}-\frac{\varepsilon}{2} .
$$

Moreover, since the map $\Pi \rightarrow\left\langle\varphi_{0}, H_{g}(\Pi) \varphi_{0}\right\rangle$, for fixed $\varphi_{0}$, is continuous in $\Pi$ (it is just a quadratic function in $\Pi$ ), there exists $\delta>0$ such that

$$
\left\langle\varphi_{0}, H_{g}(\Pi) \varphi_{0}\right\rangle \leq \Sigma_{R}-\frac{\varepsilon}{4}
$$


for all $\Pi$ with $\left|\Pi-\Pi_{0}\right| \leq \delta$. Next, we choose $f \in L^{2}\left(B_{\delta}\left(\Pi_{0}\right)\right)$ (where $B_{\delta}\left(\Pi_{0}\right)$ denotes the ball of radius $\delta$ around $\left.\Pi_{0}\right)$ with $\|f\|=1$, and we define $\varphi \in L^{2}\left(\mathbb{R}^{3}, \mathrm{~d} \Pi ; L^{2}\left(\mathbb{R}^{3}\right.\right.$, $\mathrm{d} x) \otimes \mathcal{F}$ ) by

$$
\varphi(\Pi)=f(\Pi) \varphi_{0}
$$

From (50), we obtain that

$$
\left\langle\varphi, H_{g} \varphi\right\rangle=\int \mathrm{d} \Pi\left\langle\varphi(\Pi), H_{g}(\Pi) \varphi(\Pi)\right\rangle \leq\left(\Sigma_{R}-\varepsilon / 4\right),
$$

because $\|f\|=1$. Since $\varphi \in \mathcal{D}_{R}$ (which is clear from the construction of $\varphi$ ), this contradicts the definition of $\Sigma_{R}$.

In the next proposition we prove the existence of a simple ground state for $H_{g}(\Pi)$, provided the energy is lower than a threshold energy $\Sigma$ and the coupling constant is small enough.

Proposition 2. Assume Hypotheses $(H O)$ and (HI) are satisfied. Fix $\beta<1$ and choose $\Sigma<\min \left(\Sigma_{\beta}, \Sigma_{\text {ion }}\right)$ (see Lemma 1 for the definition of $\Sigma_{\beta}$ and $\left.\Sigma_{\text {ion }}\right)$. Then, for $g$ suffciently small (depending on $\beta$ and $\Sigma$ ), $E_{g}(\Pi)=\inf \sigma\left(H_{g}(\Pi)\right)$ is a simple eigenvalue of $H_{g}(\Pi)$, provided that $E_{g}(\Pi) \leq \Sigma$.

Remark. Since $E_{g}(\Pi) \leq E_{0}^{\text {at }}+\Pi^{2} / 2 M$ it suffices that $E_{0}^{\text {at }}+\Pi^{2} / 2 M \leq \Sigma$ and that $g$ is small enough.

Proof. The proof is very similar to the proof of Theorem 4 in [FGS04]. For completeness we repeat the main ideas, but we omit details. In order to prove the proposition, we consider the modified Hamiltonian $H_{\text {mod }}$ (defined in Sect. 4.2) given, on the fiber with fixed total momentum $\Pi$, by

$$
H_{\text {mod }}(\Pi)=\frac{(\Pi-\mathrm{d} \Gamma(k))^{2}}{2 M}+\mathrm{d} \Gamma(\omega)+\frac{p^{2}}{2 m}+V(x)+g \phi\left(F_{x}\right),
$$

where the dispersion law $\omega(k)$ (with $\omega(k)=|k|$ if $|k| \geq \sigma$ and $\omega(k) \geq \sigma / 2$ for all $k$ ) is assumed to satisfy Hypothesis (H2) of Sect. 4.2. Set $E_{\bmod }(\Pi)=\inf \sigma\left(H_{\bmod }(\Pi)\right)$. Note that $H_{\text {mod }}(\Pi)$ and $H_{g}(\Pi)$ act identically on the range of $\Gamma\left(\chi_{i}\right)$, the orthogonal projection onto the subspace of vectors without soft bosons.

The proof of the proposition is divided into four steps.

1) Suppose $E_{g}(\Pi) \leq \Sigma$. Then, for sufficiently small $g$ (depending on $\beta$ and $\Sigma$ ), we have that

$$
\inf _{|k| \geq \varepsilon} E_{g}(\Pi-k)+|k|-E_{g}(\Pi)>0
$$

for every $\varepsilon>0$. This inequality follows by perturbation of the free Hamiltonian (see Lemma 35 in [FGS04] for details).

2) $E_{g}(\Pi)=E_{\text {mod }}(\Pi)$. Moreover, if $\psi$ is an eigenvector of $H_{g}(\Pi)$ (or of $H_{\text {mod }}(\Pi)$ ) corresponding to the eigenvalue $E_{g}(\Pi)$, then $\psi \in \operatorname{Ran} \Gamma\left(\chi_{i}\right)$. In particular, $\psi$ is an eigenvector of $H_{g}(\Pi)$ corresponding to the eigenvalue $E_{g}(\Pi)$ if and only if $\psi$ is an eigenvector of $H_{\bmod }(\Pi)$ corresponding to the eigenvalue $E_{\bmod }(\Pi)=E_{g}(\Pi)$. 
In order to prove these statements note that the Hamiltonians $H_{g}(\Pi)$ and $H_{\text {mod }}(\Pi)$ act on the fiber space $\mathcal{H}_{\Pi}=L^{2}\left(\mathbb{R}^{3}\right) \otimes \mathcal{F}=L^{2}\left(\mathbb{R}^{3}\right) \otimes \mathcal{F}_{i} \otimes \mathcal{F}_{s}$, where $\mathcal{F}_{s}$ is the Fock space of the soft bosons, $\mathcal{F}_{s}=\oplus_{n \geq 0} L_{s}^{2}\left(B_{\sigma}(0)^{\times n} ; \mathrm{d} k_{1} \ldots \mathrm{d} k_{n}\right)$. Thus

$$
\mathcal{H}_{\Pi} \simeq \bigoplus_{n \geq 0} L_{s}^{2}\left(B_{\sigma}(0)^{\times n}, \mathrm{~d} k_{1} \ldots \mathrm{d} k_{n} ; L^{2}\left(\mathbb{R}^{3}\right) \otimes \mathcal{F}_{i}\right)=: \bigoplus_{n \geq 0} \mathcal{H}_{\Pi}^{(n)}
$$

The restriction of $H_{g}(\Pi)$ to the subspace $\mathcal{H}_{\Pi}^{(n)}$ with exactly $n$ soft bosons is given by

$$
\left(H_{g}(\Pi) \psi\right)\left(k_{1}, \ldots, k_{n}\right)=H_{\Pi}\left(k_{1}, \ldots, k_{n}\right) \psi\left(k_{1}, \ldots, k_{n}\right)
$$

with

$$
\begin{aligned}
H_{\Pi}\left(k_{1}, \ldots, k_{n}\right)= & \frac{\left(\Pi-\mathrm{d} \Gamma(k)-\sum_{j=1}^{n} k_{j}\right)^{2}}{2 M}+\mathrm{d} \Gamma(|k|)+\sum_{j=1}^{n}\left|k_{j}\right|+\frac{p^{2}}{2 m} \\
& +V(x)+g \phi\left(F_{x}\right) \\
= & H_{g}\left(\Pi-\sum_{j=1}^{n} k_{j}\right)+\sum_{j=1}^{n}\left|k_{j}\right| \geq E_{g}\left(\Pi-\sum_{j=1}^{n} k_{j}\right)+\left|\sum_{j=1}^{n} k_{j}\right| \\
> & E_{g}(\Pi) \quad \text { if }\left(k_{1}, \ldots k_{n}\right) \neq(0, \ldots 0) .
\end{aligned}
$$

In the last inequality we used the result of part (1). This proves that

$$
\begin{aligned}
E_{g}(\Pi) & =\inf \sigma\left(H_{g}(\Pi)\right)=\inf \sigma\left(\left.H_{g}(\Pi)\right|_{L^{2}\left(\mathbb{R}^{3}\right) \otimes \mathcal{F}_{i}}\right) \\
& =\inf \sigma\left(\left.H_{\bmod }(\Pi)\right|_{L^{2}\left(\mathbb{R}^{3}\right) \otimes \mathcal{F}_{i}}\right) \geq E_{\bmod }(\Pi) .
\end{aligned}
$$

Since $H_{g}(\Pi) \leq H_{\bmod }(\Pi)$, we conclude that $E_{g}(\Pi)=E_{\bmod }(\Pi)$. Equation (54) also proves that eigenvectors of $H_{g}(\Pi)$ corresponding to the energy $E_{g}(\Pi)$, if they exist, belong to the range of $\Gamma\left(\chi_{i}\right)$. That the same is true for eigenvectors of $H_{\bmod }(\Pi)$ corresponding to the energy $E_{g}(\Pi)$ follows from an inequality for $H_{\bmod }(\Pi)$ analogous to $(54)$.

3) If $E_{g}(\Pi) \leq \Sigma$, and for $g$ sufficiently small (depending on $\beta$ and $\Sigma$ ) we have that

$$
\Delta(\Pi)=\inf _{k} E_{g}(\Pi-k)+\omega(k)-E_{g}(\Pi)>0 .
$$

For $|k|>\sigma / 4$, this follows from part (1) (because $\omega(k) \geq|k|)$, while for $|k| \leq \sigma / 4$, this inequality follows from $E_{g}(\Pi-k)+\omega(k)-E_{g}(\Pi)=E_{g}(\Pi-k)+|k|-E_{g}(\Pi)+$ $(\omega(k)-|k|) \geq \sigma / 4$, by (53) and by construction of $\omega$ (see Sect. 4.2).

4)

$$
\inf \sigma_{\mathrm{ess}}\left(H_{\mathrm{mod}}(\Pi)\right) \geq \min \left(E_{g}(\Pi)+\Delta(\Pi), \Sigma_{\bmod }(\Pi)\right)
$$

where $\Sigma_{\text {mod }}(\Pi)$ is defined like $\Sigma_{\text {ion }}$ with $H_{g}$ replaced by $H_{\text {mod }}(\Pi)$. (Recall from 2) that $E_{\bmod }(\Pi)=E_{g}(\Pi)$ ). 
The proof of part 4) is very similar to the proof of Lemma 36 in [FGS04] with a small modification at the beginning. We first need to localize with respect to the relative coordinate $x$. That is, we choose $J_{0}, J_{\infty} \in C^{\infty}\left(\mathbb{R}^{3},[0,1]\right)$ with $J_{0}(x)=1$ for $|x| \leq 1$, $J_{0}(x)=0$ for $|x| \geq 2$ and $J_{0}^{2}+J_{\infty}^{2}=1$. Let $J_{\sharp, R}(x)=J_{\sharp}(x / R)$. Then

$$
H_{\text {mod }}(\Pi)=J_{0, R} H_{\text {mod }}(\Pi) J_{0, R}+J_{\infty, R} H_{\bmod }(\Pi) J_{\infty, R}+O\left(R^{-2}\right)
$$

as $R \rightarrow \infty$. As in [FGS04], one shows that

$$
J_{0, R} H_{\text {mod }}(\Pi) J_{0, R} \geq J_{0, R}^{2}\left(E_{g}(\Pi)+\Delta(\Pi)\right)+K
$$

with $K$ relatively compact w.r.t. $H_{\bmod }(\Pi)$, while

$$
J_{\infty, R} H_{\bmod }(\Pi) J_{\infty, R} \geq J_{\infty, R}^{2} \Sigma_{\text {ion }}(\Pi)+o(1)
$$

as $R \rightarrow \infty$ follows from the definition of $\Sigma_{\bmod }(\Pi)$. Part 4$)$ follows from these estimates applied to the r.h.s. of (58).

Along with 1) and 2), and since $\Sigma_{\bmod }(\Pi) \geq \Sigma_{\text {ion }}$ for every $\Pi$ (see (49) and its proof), this proves that $E_{g}(\Pi)$ is an eigenvalue of $\bar{H}_{g}(\Pi)$, provided that $E_{g}(\Pi) \leq \Sigma$ and $g$ is sufficiently small (depending on $\Sigma$ ). The proof of the fact that $E_{g}(\Pi)$ is a simple eigenvalue is given in Corollary 6 , below.

From now on, for fixed $\beta<1$ and $\Pi$ such that $E_{g}(\Pi) \leq \Sigma<\min \left(\Sigma_{\beta}, \Sigma_{\text {ion }}\right)$, we denote by $\psi_{\Pi}$ the unique (up to a phase) normalized ground state vector of $H_{g}(\Pi)$. The vector $\psi_{\Pi}$ is called a dressed atom state with fixed total momentum $\Pi$. The space of dressed atom wave packets, $\mathcal{H}_{\text {das }} \subset \mathcal{H}$, is defined by

$$
T \mathcal{H}_{\text {das }}=\left\{\psi \in L^{2}\left(\left\{\Pi: E_{g}(\Pi) \leq \Sigma\right\} ; L^{2}\left(\mathbb{R}^{3}, \mathrm{~d} x\right) \otimes \mathcal{F}\right): \psi(\Pi) \in\left\langle\psi_{\Pi}\right\rangle\right\},
$$

where $\left\langle\psi_{\Pi}\right\rangle$ is the one-dimensional space spanned by the vector $\psi_{\Pi} ; \mathcal{H}_{\text {das }}$ is a closed linear subspace left invariant by the Hamiltonian $H_{g}$. In fact, $H_{g}$ commutes with the orthogonal projection $P_{\text {das }}$, onto $\mathcal{H}_{\text {das }}$. This follows from $\left(T P_{\text {das }} T^{*} \varphi\right)(\Pi)=P_{\psi_{\Pi}} \varphi(\Pi)$.

3.2. The Fermi Golden Rule. From Hypothesis (H0), and from standard results in the theory of Schrödinger operators (see [RS78]), it follows that the spectrum of

$$
H_{\mathrm{at}}=\frac{p^{2}}{2 m}+V
$$

in the negative half-axis $(-\infty, 0)$ is discrete. We denote the negative eigenvalues of $H_{\mathrm{at}}$ by $E_{0}^{\text {at }}<E_{1}^{\text {at }}<\cdots<0$. The eigenvalues $E_{j}^{\text {at }}$ can accumulate at zero only. If $H_{\text {at }}$ has no eigenvalues (a possibility which is not excluded by our assumptions), then our results (which only concern states of the system for which the electron is bound to the nucleus) are trivial. We denote the (finite) multiplicity of the eigenvalue $E_{j}^{\text {at }}$ by $m_{j}$. For fixed $j \geq 0$, we denote by $\varphi_{j, \alpha}, \alpha=1, \ldots m_{j}$, an orthonormal basis of the eigenspace of $H_{\mathrm{at}}$ corresponding to the eigenvalue $E_{j}^{\text {at }}$. By Hypothesis (H0), the lowest eigenvalue, $E_{0}^{\text {at }}$, of $H_{\text {at }}$ is simple ( $m_{0}=1$, see Remark (1) after Hypothesis (H0)). The unique (up to a phase) ground state vector of $H_{\text {at }}$ is denoted by $\varphi_{0}$.

For every fixed $\Pi$, the free Hamiltonian,

$$
H_{0}(\Pi)=\frac{(\Pi-\mathrm{d} \Gamma(k))^{2}}{2 M}+\mathrm{d} \Gamma(|k|)+H_{\mathrm{at}},
$$




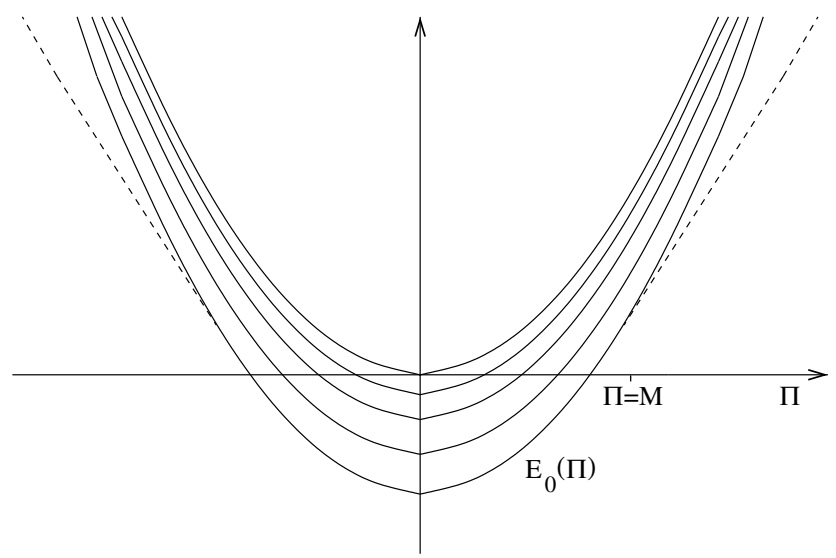

Fig. 1. Eigenvalues of the free Hamiltonian $H_{0}(\Pi)$ (the dashed curve represents inf $\sigma\left(H_{0}(\Pi)\right)$ )

has eigenvalues

$$
E_{j}(\Pi)=E_{j}^{\mathrm{at}}+\Pi^{2} / 2 M
$$

with multiplicity $m_{j}$ corresponding to the eigenvectors $\psi_{j}=\varphi_{j, \alpha} \otimes \Omega$ (where $\Omega \in \mathcal{F}$ denotes the Fock vacuum), for every $j \geq 0$. For $|\Pi| / M \leq 1, E_{0}(\Pi)=\inf \sigma\left(H_{0}(\Pi)\right)$, while all other eigenvalues $E_{j}(\Pi), j \geq 1$, are embedded in the continuous spectrum (see Fig. 1). For $|\Pi| / M>1$, all eigenvalues of $H_{0}(\Pi)$ are embedded in the continuous spectrum, and the Hamiltonian $H_{0}(\Pi)$ does not have a ground state. We restrict our attention to the physically more interesting case $|\Pi| / M \leq 1$; (this will be ensured by the condition that the total energy of the system is less than $\Sigma_{\beta=1}=E_{0}^{\text {at }}+(1 / 2) M$ and that the coupling constant $g$ is sufficiently small). One then expects the embedded eigenvalues $E_{j}(\Pi), j \geq 1$, to dissolve and to turn into resonances when the perturbation $\phi\left(F_{x}\right)$ is switched on. We prove that this is indeed the case, provided the lifetime of the resonances, as predicted by Fermi's Golden Rule in second order perturbation theory, is finite.

For given $i, j \geq 0$ and $k \in \mathbb{R}^{3}$, we define the $m_{i} \times m_{j}$ matrix

$$
\left(A_{i j}(k)\right)_{\alpha, \alpha^{\prime}}:=\left\langle\varphi_{i, \alpha}, F_{x}(k) \varphi_{j, \alpha^{\prime}}\right\rangle .
$$

For given $j \geq 0$, we then define the resonance matrix by setting

$$
\Gamma_{j}(\Pi)=\sum_{i: i \leq j} \int \mathrm{d} k A_{j i}^{*}(k) A_{j i}(k) \delta\left(\frac{(\Pi-k)^{2}}{2 M}+|k|-\frac{\Pi^{2}}{2 M}+E_{i}^{\mathrm{at}}-E_{j}^{\mathrm{at}}\right),
$$

where $\delta($.$) denotes the Dirac delta-function. Note that \Gamma_{j}(\Pi)$ is an $m_{j} \times m_{j}$ matrix. According to second order perturbation theory (Fermi's Golden Rule), the $m_{j}$ eigenvalues of $\Gamma_{j}(\Pi)$ are inverses of the lifetimes of the resonances bifurcating from the unperturbed eigenvalue $E_{j}(\Pi)=E_{j}^{\text {at }}+\Pi^{2} / 2 M$. We put

$$
\gamma_{j}(\Pi)=\inf \sigma\left(\Gamma_{j}(\Pi)\right) .
$$

Instability of the eigenvalue $E_{j}(\Pi)$ is equivalent, in second order perturbation theory, to the statement that $\gamma_{j}(\Pi)>0$. 
Hypothesis (H2). For fixed $\beta<1$ and $\Sigma<\min \left(\Sigma_{\beta}, \Sigma_{\text {ion }}\right)$, we assume that

$$
\inf \left\{\gamma_{j}(\Pi): \Pi \in \mathbb{R}^{3}, j \geq 1 \text { and } E_{j}(\Pi)<\Sigma\right\}>0 .
$$

3.3. The positive commutator. In order to prove the absence of embedded eigenvalues we use the technique of positive commutators. We prove the positivity of the commutator between the Hamiltonian $H_{g}(\Pi)$ and a suitable conjugate operator $A$. Then the absence of eigenvalues follows with the help of a virial theorem. We make use of ideas from [BFSS99], adapting them to our problem.

For fixed $j \geq 1$, we construct a suitable conjugate operator $A$, and, in Proposition 3 , we prove the positivity of the commutator $\left[H_{g}(\Pi), i A\right]$ when restricted to an energy interval $\Delta$ containing the unperturbed eigenvalue $E_{j}(\Pi)$ but no other eigenvalues of $H_{0}(\Pi)$. In Proposition 4, we then establish a commutator estimate on an energy interval around the ground state energy $E_{0}(\Pi)$ of $H_{0}(\Pi)$.

For fixed $j \geq 1$ we define

$$
P_{j}=\sum_{\alpha}\left|\varphi_{j, \alpha}\right\rangle\left\langle\varphi_{j, \alpha}\left|\otimes P_{\Omega}, \quad P_{\Omega}=\right| \Omega\right\rangle\langle\Omega| .
$$

By definition, $P_{j}$ is the orthogonal projection onto the eigenspace of $H_{0}(\Pi)$ corresponding to the eigenvalue $E_{j}(\Pi)=\Pi^{2} / 2 M+E_{j}^{\text {at }}$. Our conjugate operator is a symmetric (but not self-adjoint!) operator given by

$$
A=\mathrm{d} \Gamma(a)+i D
$$

where

$$
a=\frac{1}{2}(\hat{k} \cdot y+y \cdot \hat{k}), \quad \text { with } \hat{k}=\frac{k}{|k|}, y=i \nabla_{k},
$$

and

$$
D=g \theta P_{j} a\left(F_{x}\right) R_{\varepsilon}^{2} \bar{P}_{j}-g \theta \bar{P}_{j} R_{\varepsilon}^{2} a^{*}\left(F_{x}\right) P_{j} .
$$

In (67), we introduced the notation $\bar{P}_{j}=1-P_{j}$ and we used

$$
R_{\varepsilon}^{2}=\left(\left(H_{0}(\Pi)-E_{j}(\Pi)\right)^{2}+\varepsilon^{2}\right)^{-1}
$$

Note that $\varepsilon R_{\varepsilon}^{2} \rightarrow \delta\left(H_{0}(\Pi)-E_{j}(\Pi)\right)$ strongly, as $\varepsilon \rightarrow 0$. The real parameters $\theta$ and $\varepsilon$ will be fixed later on.

By a formal computation, the commutator $H_{g}(\Pi) i A-i A H_{g}(\Pi)$ is given by

$$
\left[H_{g}(\Pi), i A\right]=N-\frac{(\Pi-\mathrm{d} \Gamma(k))}{M} \cdot \mathrm{d} \Gamma(\hat{k})-g \phi\left(i a F_{x}\right)-\left[H_{g}(\Pi), D\right] .
$$

This expression is our definition of the operator $\left[H_{g}(\Pi), i A\right]$. In the proof of Proposition 3 we will see that $\left\langle\varphi,\left[H_{g}(\Pi), i A\right] \varphi\right\rangle$ defines a quadratic form that is bounded from below (but possibly $+\infty$ ) on vectors from the spectral subspaces $\operatorname{Ran} E_{\Delta}\left(H_{g}(\Pi)\right.$ ) of Proposition 3. On even smaller subspaces a rigorous connection between $\left[H_{g}(\Pi), i A\right]$ and $A$ will be established in the proof of the Virial Theorem, Proposition 5. 
Proposition 3. We assume that Hypotheses (HO)-(H2) are satisfied. We fix $\beta<1$ and choose $\Sigma<\min \left(\Sigma_{\beta}, \Sigma_{\text {ion }}\right)$; (see Lemma 1 for the definition of $\Sigma_{\beta}$ and $\left.\Sigma_{\text {ion }}\right)$. Moreover, we suppose that the interval $\Delta \subset(-\infty, \Sigma)$ is such that $E_{j}(\Pi) \in \Delta$ and

$$
d:=\operatorname{dist}\left(\Delta, \sigma_{p p}\left(H_{0}(\Pi)\right) \backslash\left\{E_{j}(\Pi)\right\}\right)>0 .
$$

Then, for $g>0$ sufficiently small (depending on $\beta, \Sigma$ and the distance $d$ ), one can choose $\varepsilon$ and $\theta$ such that

$$
E_{\Delta}\left(H_{g}(\Pi)\right)\left[H_{g}(\Pi), i A\right] E_{\Delta}\left(H_{g}(\Pi)\right) \geq C E_{\Delta}\left(H_{g}(\Pi)\right),
$$

with a positive constant $C$.

Remarks.

1) The choice of the parameter $\varepsilon, \theta$ and of the constant $C$ depends on the value of $g$. We can choose, for example,

$$
\theta \simeq O\left(g^{\kappa}\right) \quad \varepsilon \simeq O\left(g^{\alpha}\right) \quad C \simeq O\left(g^{2+\kappa-\alpha}\right),
$$

for $0<\kappa<\alpha<1$.

2) How small $g$ has to be chosen depends on the values of $\beta$, on $\gamma_{j}(\Pi)$, and on the distance $d$ in (70). We must have that $g \ll(1-\beta), \gamma_{j}(\Pi) \gg g^{\alpha-\kappa}$, and $\gamma_{j}(\Pi) d^{2} \gg \max \left(g^{\alpha-\kappa}, g^{1-\alpha}\right)$ (for an arbitrary choice of $\kappa, \alpha$ with $0<\kappa<\alpha<1$ ). Moreover, $g$ has to be sufficiently small, in order for Eq. (44) to hold true (and thus $g$ depends on the choice of the threshold $\Sigma$ ).

3) In this proposition, we do not need the infrared cutoff in the interaction (i.e., we can choose $\sigma=0$ ). However, the infrared cutoff is needed in the proof of Proposition 5 (the Virial Theorem), and hence in the proof of the absence of embedded eigenvalues.

Proof. Using Eq. (44), it is easy to check that, for $\Delta$ as above,

$$
\begin{gathered}
E_{\Delta}\left(H_{g}(\Pi)\right)\left(N-\frac{\Pi-\mathrm{d} \Gamma(k)}{M} \cdot \mathrm{d} \Gamma(\hat{k})\right) E_{\Delta}\left(H_{g}(\Pi)\right) \\
\quad \geq(1-\beta) E_{\Delta}\left(H_{g}(\Pi)\right)\left(1-P_{\Omega}\right) E_{\Delta}\left(H_{g}(\Pi)\right) .
\end{gathered}
$$

Thus, defining

$$
B=(1-\beta)\left(1-P_{\Omega}\right)-g \phi\left(i a F_{x}\right)-\left[H_{g}(\Pi), D\right],
$$

we conclude that

$$
E_{\Delta}\left(H_{g}(\Pi)\right)\left[H_{g}(\Pi), i A\right] E_{\Delta}\left(H_{g}(\Pi)\right) \geq E_{\Delta}\left(H_{g}(\Pi)\right) B E_{\Delta}\left(H_{g}(\Pi)\right),
$$

and it is enough to prove the positivity of the r.h.s. of the last equation to complete the proof. The advantage of working with $B$, instead of the commutator $\left[H_{g}(\Pi), i A\right]$, is that $B$ is bounded with respect to the Hamiltonian $H_{g}(\Pi)$ while $\left[H_{g}(\Pi), i A\right]$ is not (since the number operator $N$ is not bounded with respect to $\left.H_{g}(\Pi)\right)$. In order to prove that

$$
E_{\Delta}\left(H_{g}(\Pi)\right) B E_{\Delta}\left(H_{g}(\Pi)\right) \geq C E_{\Delta}\left(H_{g}(\Pi)\right),
$$

we first establish the inequality

$$
E_{\Delta}\left(H_{0}(\Pi)\right) B E_{\Delta}\left(H_{0}(\Pi)\right) \geq C E_{\Delta}\left(H_{0}(\Pi)\right) .
$$


To this end we may assume that

$$
\lambda_{0}:=\inf \sigma\left(\left.E_{\Delta}\left(H_{0}(\Pi)\right) B E_{\Delta}\left(H_{0}(\Pi)\right)\right|_{\operatorname{Ran} E_{\Delta}\left(H_{0}(\Pi)\right)}\right) \leq \frac{1-\beta}{2},
$$

for otherwise (76) holds with $C=(1-\beta) / 2$. This assumption will allow us to apply the Feshbach map with projection $P_{j}$ to the operator $E_{\Delta}\left(H_{0}(\Pi)\right) B E_{\Delta}\left(H_{0}(\Pi)\right)-\lambda_{0}$. Indeed, this operator restricted to $\operatorname{Ran} \bar{P}_{j}$ is invertible for small $g$ and $g^{2} \theta \varepsilon^{-2}$ as is shown in Step 1. Step 1 through Step 5 prepare the proof of (76).

Step 1. There exists a constant $C>0$, independent of $\Pi$ and $g$, such that

$$
\bar{P}_{j} E_{\Delta}\left(H_{0}(\Pi)\right) B E_{\Delta}\left(H_{0}(\Pi)\right) \bar{P}_{j} \geq\left(1-\beta-C\left(g+\frac{g^{2} \theta}{\varepsilon^{2}}\right)\right) \bar{P}_{j} E_{\Delta}\left(H_{0}(\Pi)\right) .
$$

In fact,

$$
\begin{aligned}
& \bar{P}_{j} E_{\Delta}\left(H_{0}(\Pi)\right) B E_{\Delta}\left(H_{0}(\Pi)\right) \bar{P}_{j} \\
& \geq(1-\beta) \bar{P}_{j} E_{\Delta}\left(H_{0}(\Pi)\right)\left(1-P_{\Omega}\right) E_{\Delta}\left(H_{0}(\Pi)\right) \bar{P}_{j} \\
& \quad+g \bar{P}_{j} E_{\Delta}\left(H_{0}(\Pi)\right) \phi\left(i a F_{x}\right) E_{\Delta}\left(H_{0}(\Pi)\right) \bar{P}_{j} \\
& \quad-g^{2} \theta \bar{P}_{j} E_{\Delta}\left(H_{0}(\Pi)\right) a^{*}\left(F_{x}\right) P_{j} a\left(F_{x}\right) R_{\varepsilon}^{2} E_{\Delta}\left(H_{0}(\Pi)\right) \bar{P}_{j} \\
& \quad-g^{2} \theta \bar{P}_{j} E_{\Delta}\left(H_{0}(\Pi)\right) R_{\varepsilon}^{2} a^{*}\left(F_{x}\right) P_{j} a\left(F_{x}\right) E_{\Delta}\left(H_{0}(\Pi)\right) \bar{P}_{j} .
\end{aligned}
$$

Applying Lemma 21 of Appendix B to bound $\phi\left(i a F_{x}\right)$, using that $P_{\Omega} \bar{P}_{j} E_{\Delta}\left(H_{0}(\Pi)\right)=$ 0 (by the choice of the interval $\Delta$ ), and that $\left\|R_{\varepsilon}^{2}\right\| \leq \varepsilon^{-2}$, inequality (78) follows easily.

Step 2. We define

$$
\begin{aligned}
\mathcal{E}= & P_{j} B P_{j}-P_{j} B \bar{P}_{j} E_{\Delta}\left(H_{0}(\Pi)\right)\left(\left.\left(B-\lambda_{0}\right)\right|_{\operatorname{Ran} \bar{P}_{j} E_{\Delta}\left(H_{0}(\Pi)\right)}\right)^{-1} \\
& \times E_{\Delta}\left(H_{0}(\Pi)\right) \bar{P}_{j} B P_{j},
\end{aligned}
$$

where $\lambda_{0}$ is defined in (77) (note that, by (77) and by the result of Step 1, the inverse on the r.h.s. of (80) is well defined, if $g$ and $g^{2} \theta / \varepsilon^{2}$ are small enough). Then we have

$$
\lambda_{0} \geq \inf \sigma\left(\left.\mathcal{E}\right|_{\operatorname{Ran} P_{j}}\right) .
$$

The proof of (81) relies on the isospectrality of the Feshbach map and can be found, for example, in [BFSS99]. This inequality says that, instead of finding a bound on the operator $B$ restricted to the range of $E_{\Delta}\left(H_{0}(\Pi)\right)$, we can study the operator $\mathcal{E}$ restricted to the much smaller range of the projection $P_{j}$ (which is finite-dimensional).

Using the assumption (77) and Eq. (78), we see that, if $g$ and $g^{2} \theta / \varepsilon^{2}$ are sufficiently small,

$$
\left.\left(B-\lambda_{0}\right)\right|_{\operatorname{Ran} \bar{P}_{j} E_{\Delta}\left(H_{0}(\Pi)\right)} \geq \frac{1-\beta}{4} .
$$

(Later, when we will choose the parameter $\theta$ and $\varepsilon$, we will make sure that $g^{2} \theta / \varepsilon^{2}$ is small enough, if $g$ is small enough). This implies that the operator $\mathcal{E}$ is bounded from below by

$$
\mathcal{E} \geq P_{j} B P_{j}-\frac{4}{1-\beta} P_{j} B \bar{P}_{j} E_{\Delta}\left(H_{0}(\Pi)\right) \bar{P}_{j} B P_{j} .
$$

Next, we study the second term on the r.h.s. of inequality (83). 
Step 3. There exists a constant $C>0$ independent of $g, \theta$ and $\varepsilon$ such that

$$
P_{j} B \bar{P}_{j} E_{\Delta}\left(H_{0}(\Pi)\right) \bar{P}_{j} B P_{j} \leq C g^{2} P_{j}+C\left(g^{2} \theta^{2}+\frac{g^{4} \theta^{2}}{\varepsilon^{2}}\right) P_{j} a\left(F_{x}\right) R_{\varepsilon}^{2} a^{*}\left(F_{x}\right) P_{j} .
$$

In order to prove this bound, we note that, for any $\psi \in \mathcal{H}$,

$$
\left\langle\psi, P_{j} B \bar{P}_{j} E_{\Delta}\left(H_{0}(\Pi)\right) \bar{P}_{j} B P_{j} \psi\right\rangle=\left\|E_{\Delta}\left(H_{0}(\Pi)\right) \bar{P}_{j} B P_{j} \psi\right\|^{2} .
$$

Furthermore

$$
\begin{aligned}
\bar{P}_{j} B P_{j} & =-g \bar{P}_{j} \phi\left(i a F_{x}\right) P_{j}-\bar{P}_{j}\left[H_{g}(\Pi)-E_{j}(\Pi), D\right] P_{j} \\
& =-g \bar{P}_{j} \phi\left(i a F_{x}\right) P_{j}+g \theta \bar{P}_{j}\left(H_{g}(\Pi)-E_{j}(\Pi)\right) \bar{P}_{j} R_{\varepsilon}^{2} a^{*}\left(F_{x}\right) P_{j},
\end{aligned}
$$

because

$$
P_{j}\left(H_{g}(\Pi)-E_{j}(\Pi)\right) P_{j}=P_{j}\left(H_{0}(\Pi)-E_{j}(\Pi)\right) P_{j}+g P_{j} \phi\left(F_{x}\right) P_{j}=0 .
$$

Hence we find that

$$
\begin{aligned}
E_{\Delta}\left(H_{0}(\Pi)\right) \bar{P}_{j} B P_{j} \psi= & -g E_{\Delta}\left(H_{0}(\Pi)\right) \bar{P}_{j} \phi\left(i a F_{x}\right) P_{j} \psi \\
& +g \theta \bar{P}_{j} E_{\Delta}\left(H_{0}(\Pi)\right)\left(H_{0}(\Pi)-E_{j}(\Pi)\right) R_{\varepsilon}^{2} a^{*}\left(F_{x}\right) P_{j} \psi \\
& +g^{2} \theta \bar{P}_{j} E_{\Delta}\left(H_{0}(\Pi)\right) \phi\left(F_{x}\right) \bar{P}_{j} R_{\varepsilon}^{2} a^{*}\left(F_{x}\right) P_{j} \psi .
\end{aligned}
$$

Using Lemma 21 and the bound $\left\|\left(H_{0}(\Pi)-E_{j}(\Pi)\right) R_{\varepsilon}\right\| \leq 1$, we conclude that

$$
\left\|E_{\Delta}\left(H_{0}(\Pi)\right) \bar{P}_{j} B P_{j} \psi\right\| \leq C g\left\|P_{j} \psi\right\|+C\left(g \theta+g^{2} \theta / \varepsilon\right)\left\|R_{\varepsilon} a^{*}\left(F_{x}\right) P_{j} \psi\right\| .
$$

Taking the square of this inequality, we obtain (84).

Step 4. We show that

$$
P_{j} B P_{j}=2 g^{2} \theta P_{j} a\left(F_{x}\right) R_{\varepsilon}^{2} a^{*}\left(F_{x}\right) P_{j} .
$$

Using that $P_{j}\left(1-P_{\Omega}\right) P_{j}=0$ and $P_{j} \phi\left(i a G_{x}\right) P_{j}=0$, we easily find that

$$
P_{j} B P_{j}=g \theta P_{j} H_{g}(\Pi) \bar{P}_{j} R_{\varepsilon}^{2} a^{*}\left(F_{x}\right) P_{j}+g \theta P_{j} a\left(F_{x}\right) R_{\varepsilon}^{2} \bar{P}_{j} H_{g}(\Pi) P_{j} .
$$

Writing $H_{g}(\Pi)=H_{0}(\Pi)+g \phi\left(F_{x}\right)$, using that $P_{j}$ commutes with $H_{0}(\Pi)$, and that $P_{j} a^{*}\left(F_{x}\right)=a\left(F_{x}\right) P_{j}=0$ we find

$$
P_{j} B P_{j}=g \theta P_{j} a\left(F_{x}\right) \bar{P}_{j} R_{\varepsilon}^{2} a^{*}\left(F_{x}\right) P_{j}+g \theta P_{j} a\left(F_{x}\right) R_{\varepsilon}^{2} \bar{P}_{j} a^{*}\left(F_{x}\right) P_{j} .
$$

Equation (89) now follows writing $\bar{P}_{j}=1-P_{j}$ and using that $P_{j} a\left(F_{x}\right) P_{j}=$ $P_{j} a^{*}\left(F_{x}\right) P_{j}=0$.

From Step 3 and Step 4 and from (83) we get

$$
\mathcal{E} \geq\left(2 g^{2} \theta-C\left(g^{2} \theta^{2}+\frac{g^{4} \theta^{2}}{\varepsilon^{2}}\right)\right) P_{j} a\left(F_{x}\right) R_{\varepsilon}^{2} a^{*}\left(F_{x}\right) P_{j}-C g^{2} P_{j}
$$

for a constant $C$ independent of $g, \theta$ and $\varepsilon$. 
Step 5. Next, we claim that

$$
P_{j} a\left(F_{x}\right) R_{\varepsilon}^{2} a^{*}\left(F_{x}\right) P_{j} \geq \frac{\gamma_{j}(\Pi)}{\varepsilon}\left(1+o_{\varepsilon}(1)\right) P_{j},
$$

where $o_{\varepsilon}(1) \rightarrow 0$, as $\varepsilon \rightarrow 0$.

In order to prove (93), we use the pull-through formula for $a(q) R_{\varepsilon}^{2}$ and the fact that $a(q) P_{j}=0$. This yields

$$
\begin{array}{rl}
P_{j} & a\left(F_{x}\right) R_{\varepsilon}^{2} a^{*}\left(F_{x}\right) P_{j} \\
= & \int \mathrm{d} q \mathrm{~d} q^{\prime} P_{j} \overline{F_{x}(q)} a(q) R_{\varepsilon}^{2} F_{x}\left(q^{\prime}\right) a^{*}\left(q^{\prime}\right) P_{j} \\
= & \int \mathrm{d} q \mathrm{~d} q^{\prime} P_{j} \overline{F_{x}(q)}\left(\left(\frac{(\Pi-q-\mathrm{d} \Gamma(k))^{2}}{2 M}+|q|+\mathrm{d} \Gamma(|k|)+H_{\mathrm{at}}-E_{j}(\Pi)\right)^{2}+\varepsilon^{2}\right)^{-1} \\
& \times F_{x}\left(q^{\prime}\right) a(q) a^{*}\left(q^{\prime}\right) P_{j} \\
= & \int \mathrm{d} q P_{j} \overline{F_{x}(q)}\left(\left(\frac{(\Pi-q-\mathrm{d} \Gamma(k))^{2}}{2 M}+|q|+\mathrm{d} \Gamma(|k|)+H_{\mathrm{at}}-E_{j}(\Pi)\right)^{2}+\varepsilon^{2}\right)^{-1} \\
& \times F_{x}(q) P_{j} .
\end{array}
$$

Next, we write $P_{j}=P_{j}^{\text {at }} \otimes P_{\Omega}$, where $P_{j}^{\text {at }}=\sum_{\alpha=1}^{m_{j}}\left|\phi_{j, \alpha}\right\rangle\left\langle\phi_{j, \alpha}\right|$ is the orthogonal projection onto the eigenspace of $H_{\text {at }}$ corresponding to the eigenvalue $E_{j}^{\text {at }}$. Then we obtain that

$$
\begin{aligned}
& P_{j} a\left(F_{x}\right) R_{\varepsilon}^{2} a^{*}\left(F_{x}\right) P_{j} \\
& =\left\{\int \mathrm{d} q P_{j}^{\mathrm{at}} \overline{F_{x}(q)}\left(\left(\frac{(\Pi-q)^{2}}{2 M}+|q|-\frac{\Pi^{2}}{2 M}+H_{\mathrm{at}}-E_{j}^{\mathrm{at}}\right)^{2}+\varepsilon^{2}\right)^{-1}\right. \\
& \left.\times F_{x}(q) P_{j}^{\text {at }}\right\} \otimes P_{\Omega} .
\end{aligned}
$$

All operators involved in the expression on the r.h.s. of (95) act trivially on the Fock space, and we get the lower bound

$$
\begin{aligned}
& \int \mathrm{d} q P_{j}^{\mathrm{at}} \overline{F_{x}(q)}\left(\left(\frac{(\Pi-q)^{2}}{2 M}+|q|-\frac{\Pi^{2}}{2 M}+H_{\mathrm{at}}-E_{j}^{\mathrm{at}}\right)^{2}+\varepsilon^{2}\right)^{-1} F_{x}(q) P_{j}^{\mathrm{at}} \\
& \geq \sum_{m \leq j} \int \mathrm{d} q P_{j}^{\mathrm{at}} \overline{F_{x}(q)} P_{m}^{\mathrm{at}}\left(\left(\frac{(\Pi-q)^{2}}{2 M}+|q|-\frac{\Pi^{2}}{2 M}+H_{\mathrm{at}}-E_{j}^{\mathrm{at}}\right)^{2}+\varepsilon^{2}\right)^{-1} \\
& \times P_{m}^{\text {at }} F_{x}(q) P_{j}^{\text {at }} \\
& =\sum_{m \leq j} \sum_{\alpha, \alpha^{\prime}} \int \mathrm{d} q\left(\left(\frac{(\Pi-q)^{2}}{2 M}+|q|-\frac{\Pi^{2}}{2 M}+E_{m}^{\mathrm{at}}-E_{j}^{\mathrm{at}}\right)^{2}+\varepsilon^{2}\right)^{-1} \\
& \times\left(A_{m j}^{*}(q) A_{m j}(q)\right)_{\alpha, \alpha^{\prime}}\left|\varphi_{j, \alpha}\right\rangle\left\langle\varphi_{j, \alpha^{\prime}}\right| .
\end{aligned}
$$


Using that $\varepsilon\left(x^{2}+\varepsilon^{2}\right)^{-1}=\delta(x)+o_{\varepsilon}(1)$, as $\varepsilon \rightarrow 0$, and recalling the definition of the matrix $\Gamma_{j}(\Pi)$, we find that

$$
\begin{aligned}
\left\langle\psi, P_{j} a\left(F_{x}\right) R_{\varepsilon}^{2} a^{*}\left(F_{x}\right) P_{j} \psi\right\rangle & \geq \frac{1}{\varepsilon}\left(1+o_{\varepsilon}(1)\right)\left\langle\psi, P_{j} \Gamma_{j}(\Pi) P_{j} \psi\right\rangle \\
& \geq \frac{\gamma_{j}(\Pi)}{\varepsilon}\left(1+o_{\varepsilon}(1)\right)\left\|P_{j} \psi\right\|^{2} .
\end{aligned}
$$

This proves Eq. (93).

Proof of Eq. (76). From (92) and (93), we derive that

$$
\mathcal{E} \geq\left(\frac{\gamma_{j}(\Pi)}{\varepsilon}\left(2 g^{2} \theta-C\left(g^{2} \theta^{2}+\frac{g^{4} \theta^{2}}{\varepsilon^{2}}\right)\right)\left(1+o_{\varepsilon}(1)\right)-C g^{2}\right) P_{j} .
$$

Choosing $\varepsilon=g^{\alpha}$ and $\theta=g^{\kappa}$, with $0<\kappa<\alpha<1$, we get

$$
\mathcal{E} \geq \gamma_{j}(\Pi) g^{2+\kappa-\alpha} P_{j},
$$

for $g$ sufficiently small. Note that, with this choice of $\varepsilon$ and $\theta, g^{2} \theta / \varepsilon^{2}=g^{2+\kappa-2 \alpha} \ll 1$, and thus (82) is satisfied, if $g$ is small enough. From (77) and (81) we then get that

$$
E_{\Delta}\left(H_{0}(\Pi)\right) B E_{\Delta}\left(H_{0}(\Pi)\right) \geq \gamma_{j}(\Pi) g^{2+\kappa-\alpha} E_{\Delta}\left(H_{0}(\Pi)\right)
$$

which proves (76).

Proof of Eq. (75). To prove (75), and hence complete the proof of the proposition, we must replace $E_{\Delta}\left(H_{0}(\Pi)\right)$ by the spectral projection $E_{\Delta}\left(H_{g}(\Pi)\right)$ of the full Hamiltonian $H_{g}(\Pi)$.

Given an interval $\Delta \subset(-\infty, \Sigma)$ with $E_{j} \in \Delta$ and

$$
\operatorname{dist}\left(\Delta, \sigma_{\mathrm{pp}}\left(H_{0}(\Pi)\right) \backslash\left\{E_{j}\right\}\right)>0,
$$

we choose an interval $\widetilde{\Delta} \subset(-\infty, \Sigma)$ such that $\Delta \subset \widetilde{\Delta}$, and

$$
\operatorname{dist}\left(\widetilde{\Delta}, \sigma_{\mathrm{pp}}\left(H_{0}(\Pi)\right) \backslash\left\{E_{j}\right\}\right)>0
$$

and with $\delta=\operatorname{dist}\left(\Delta, \widetilde{\Delta}^{\mathrm{c}}\right)>0$. Furthermore, we choose a function $\chi \in C^{\infty}(\mathbb{R})$ with the property that $\chi=1$ on $\Delta$ and $\chi=0$ on $\widetilde{\Delta}^{\mathrm{c}}$. We can assume that $\left|\chi^{\prime}(s)\right| \leq C \delta^{-1}$. Applying (100) with $\Delta$ replaced by $\widetilde{\Delta}$, and multiplying the resulting inequality with $\chi\left(H_{0}(\Pi)\right)$ we get

$$
\chi\left(H_{0}(\Pi)\right) B \chi\left(H_{0}(\Pi)\right) \geq \gamma_{j}(\Pi) g^{2+\kappa-\alpha} \chi^{2}\left(H_{0}(\Pi)\right) .
$$

Setting $\chi:=\chi\left(H_{g}(\Pi)\right)$ and $\chi_{0}:=\chi\left(H_{0}(\Pi)\right)$, we have that

$$
\chi B \chi=\chi_{0} B \chi_{0}+\left(\chi-\chi_{0}\right) B \chi_{0}+\chi_{0} B\left(\chi-\chi_{0}\right)+\left(\chi-\chi_{0}\right) B\left(\chi-\chi_{0}\right) .
$$

Using

$$
\left(\chi-\chi_{0}\right) B \chi_{0}+\chi_{0} B\left(\chi-\chi_{0}\right) \geq-(1 / 2) \chi_{0} B \chi_{0}-2\left(\chi-\chi_{0}\right)|B|\left(\chi-\chi_{0}\right)
$$

we find that

$$
\chi B \chi \geq 1 / 2 \chi_{0} B \chi_{0}-3\left(\chi-\chi_{0}\right)|B|\left(\chi-\chi_{0}\right) .
$$


Next we use that

$$
\begin{aligned}
\chi-\chi_{0} & =\int \mathrm{d} z \partial_{\bar{z}} \tilde{\chi}(z)\left(\frac{1}{H_{g}(\Pi)-z}-\frac{1}{H_{0}(\Pi)-z}\right) \\
& =g \int \mathrm{d} z \partial_{\bar{z}} \widetilde{\chi}(z) \frac{1}{H_{g}(\Pi)-z} \phi\left(F_{x}\right) \frac{1}{H_{0}(\Pi)-z} .
\end{aligned}
$$

From the definition of $B$ it follows that

$$
\left\|\left(H_{0}(\Pi)-z_{1}\right)^{-1}|B|\left(H_{g}(\Pi)-z\right)^{-1}\right\| \leq C\left(1+g \theta \varepsilon^{-2}\right)=C\left(1+g^{1+\kappa-2 \alpha}\right),
$$

with the choice $\varepsilon=g^{\alpha}, \theta=g^{\kappa}$. This implies that

$$
\left\langle\psi,\left(\chi-\chi_{0}\right)|B|\left(\chi-\chi_{0}\right) \psi\right\rangle \leq C\left(g^{2}+g^{3+\kappa-2 \alpha}\right)\|\psi\|^{2},
$$

where the constant $C$ depends on $\delta$ ( $C$ is proportional to $\left.\delta^{-2}\right)$. Thus, with (106),

$$
\begin{aligned}
\chi B \chi & \geq(1 / 2) \gamma_{j}(\Pi) g^{2+\kappa-\alpha} \chi_{0}^{2}-C\left(g^{2}+g^{3+\kappa-2 \alpha}\right) \\
& \geq(1 / 2) \gamma_{j}(\Pi) g^{2+\kappa-\alpha} \chi^{2}-C\left(g^{2}+g^{3+\kappa-2 \alpha}\right) .
\end{aligned}
$$

Since $0<\kappa<\alpha<1$, we have $g^{2+\kappa-\alpha} \gg g^{2}$ and $g^{2+\kappa-\alpha} \gg g^{3+\kappa-2 \alpha}$. Therefore, multiplying from the left and the right with $E_{\Delta}\left(H_{g}(\Pi)\right)$, and choosing $g$ small enough, we find that

$$
E_{\Delta}\left(H_{g}(\Pi)\right) B E_{\Delta}\left(H_{g}(\Pi)\right) \geq C E_{\Delta}\left(H_{g}(\Pi)\right),
$$

for some positive constant $C$, which, with (74), completes the proof of the proposition.

Proposition 3 and Proposition 5, below, prove absence of embedded eigenvalues of $H_{g}(\Pi)$ on $(-\infty, \Sigma)$ with the exception of a small interval around the ground state energy, inf $\sigma\left(H_{0}(\Pi)\right)$. Absence of embedded eigenvalues near the ground state energy follows from our next proposition.

Recall from (66) the notation

$$
a:=\frac{1}{2}(\hat{k} \cdot y+y \cdot \hat{k})
$$

with $\hat{k}=k /|k|, y=i \nabla_{k}$. A formal calculation shows that

$$
\left[H_{g}(\Pi), i \mathrm{~d} \Gamma(a)\right]=N-\frac{\Pi-\mathrm{d} \Gamma(k)}{M} \cdot \mathrm{d} \Gamma(\hat{k})-g \phi\left(i a F_{x}\right) .
$$

This expression is our definition of the operator $\left[H_{g}(\Pi), i \mathrm{~d} \Gamma(a)\right]$. The remarks after (69) apply equally to the connection between $\left[H_{g}(\Pi), i \mathrm{~d} \Gamma(a)\right]$ and $\mathrm{d} \Gamma(a)$.

Proposition 4. Assume Hypotheses (HO)-(H1). Fix $\beta<1$, and choose $\Sigma<\min \left(\Sigma_{\beta}\right.$, $\left.\Sigma_{\text {ion }}\right)$, with $\Sigma_{\beta}=E_{0}^{a t}+(M / 2) \beta^{2}$. Suppose the interval $\Delta \subset(-\infty, \Sigma)$ is such that

$$
\Delta \subset\left(-\infty, E_{1}(\Pi)\right) \text { and } d=\operatorname{dist}\left(\Delta, E_{1}(\Pi)\right)>0 .
$$

(Recall that $E_{1}(\Pi)$ denotes the first excited eigenvalue of the free Hamiltonian $H_{0}(\Pi)$ ). Then, if $g \geq 0$ is sufficiently small (depending on $\beta, \Sigma$ and $d$ ), there exists $C>0$ such that

$$
\begin{aligned}
& E_{\Delta}\left(H_{g}(\Pi)\right)\left[H_{g}(\Pi), i \mathrm{~d} \Gamma(a)\right] E_{\Delta}\left(H_{g}(\Pi)\right) \\
& \quad \geq(1-\beta) E_{\Delta}\left(H_{g}(\Pi)\right)\left(1-P_{\varphi_{0} \otimes \Omega}\right) E_{\Delta}\left(H_{g}(\Pi)\right)-C g E_{\Delta}\left(H_{g}(\Pi)\right) .
\end{aligned}
$$


Proof. By definition of $\left[H_{g}(\Pi), i \mathrm{~d} \Gamma(a)\right]$,

$$
\begin{aligned}
& E_{\Delta}\left(H_{g}(\Pi)\right)\left[H_{g}(\Pi), i \mathrm{~d} \Gamma(a)\right] E_{\Delta}\left(H_{g}(\Pi)\right) \\
& \quad \geq(1-\beta) E_{\Delta}\left(H_{g}(\Pi)\right) N E_{\Delta}\left(H_{g}(\Pi)\right) \\
& \quad-g E_{\Delta}\left(H_{g}(\Pi)\right) \phi\left(i a F_{x}\right) E_{\Delta}\left(H_{g}(\Pi)\right) \\
& \quad \geq(1-\beta-C g) E_{\Delta}\left(H_{g}(\Pi)\right)-(1-\beta) E_{\Delta}\left(H_{g}(\Pi)\right) P_{\Omega} E_{\Delta}\left(H_{g}(\Pi)\right) .
\end{aligned}
$$

Here we use that, by Hypothesis (H1), $\left\|E_{\Delta}\left(H_{g}(\Pi)\right)|\Pi-\mathrm{d} \Gamma(k)| / M\right\| \leq \beta$, and that, by Lemma 21, $\left\|\phi\left(i a F_{x}\right) E_{\Delta}\left(H_{g}(\Pi)\right)\right\| \leq C$.

Next, we note that

$$
\begin{aligned}
E_{\Delta}\left(H_{g}(\Pi)\right) P_{\Omega} E_{\Delta}\left(H_{g}(\Pi)\right)= & E_{\Delta}\left(H_{g}(\Pi)\right)\left(\chi\left(H_{\mathrm{at}}=E_{0}^{\mathrm{at}}\right) \otimes P_{\Omega}\right) E_{\Delta}\left(H_{g}(\Pi)\right) \\
& +E_{\Delta}\left(H_{g}(\Pi)\right)\left(\chi\left(H_{\mathrm{at}} \geq E_{1}^{\mathrm{at}}\right) \otimes P_{\Omega}\right) E_{\Delta}\left(H_{g}(\Pi)\right) \\
= & E_{\Delta}\left(H_{g}(\Pi)\right) P_{\varphi_{0} \otimes \Omega} E_{\Delta}\left(H_{g}(\Pi)\right) \\
& +E_{\Delta}\left(H_{g}(\Pi)\right) \chi\left(H_{0}(\Pi) \geq E_{1}(\Pi)\right) E_{\Delta}\left(H_{g}(\Pi)\right),
\end{aligned}
$$

where $\varphi_{0}$ is the unique (up to a phase) ground state vector of the atomic Hamiltonian $H_{\text {at }}$ (the fact that the ground state vector of $H_{\text {at }}$ is unique follows from Hypothesis ( $\left.\mathrm{H} 0\right)$, see Remark (1) after Hypothesis (H0)). Next we choose a function $\chi \in C^{\infty}(\mathbb{R})$ with $\chi(s)=0$ for $s \leq E_{1}(\Pi)-d$ and $\chi(s)=1$ for $s \geq E_{1}(\Pi)$. Equation (115) then implies that

$$
\begin{aligned}
& E_{\Delta}\left(H_{g}(\Pi)\right) P_{\Omega} E_{\Delta}\left(H_{g}(\Pi)\right) \\
& \quad \leq E_{\Delta}\left(H_{g}(\Pi)\right) P_{\varphi_{0} \otimes \Omega} E_{\Delta}\left(H_{g}(\Pi)\right)+E_{\Delta}\left(H_{g}(\Pi)\right) \chi\left(H_{0}(\Pi)\right) E_{\Delta}\left(H_{g}(\Pi)\right) .
\end{aligned}
$$

Note that

$$
\begin{aligned}
\chi\left(H_{0}(\Pi)\right)-\chi\left(H_{g}(\Pi)\right) & =\int \mathrm{d} z \partial_{\bar{z}} \tilde{\chi}(z)\left(\frac{1}{H_{0}(\Pi)-z}-\frac{1}{H_{g}(\Pi)-z}\right) \\
& =C g \int \mathrm{d} z \partial_{\bar{z}} \tilde{\chi}(z) \frac{1}{H_{0}(\Pi)-z} \phi\left(F_{x}\right) \frac{1}{H_{g}(\Pi)-z} .
\end{aligned}
$$

Since, by definition of the interval $\Delta, \chi\left(H_{g}(\Pi)\right) E_{\Delta}\left(H_{g}(\Pi)\right)=0$, we find that

$$
\begin{aligned}
E_{\Delta}\left(H_{g}(\Pi)\right) \chi\left(H_{0}(\Pi)\right) E_{\Delta}\left(H_{g}(\Pi)\right)= & E_{\Delta}\left(H_{g}(\Pi)\right) \\
& \times\left(\chi\left(H_{g}(\Pi)\right)-\chi\left(H_{0}(\Pi)\right)\right) E_{\Delta}\left(H_{g}(\Pi)\right) \\
\leq & C g E_{\Delta}\left(H_{g}(\Pi)\right) .
\end{aligned}
$$

With (114) and (116), this shows that

$$
\begin{aligned}
& E_{\Delta}\left(H_{g}(\Pi)\right)\left[H_{g}(\Pi), i \mathrm{~d} \Gamma(a)\right] E_{\Delta}\left(H_{g}(\Pi)\right) \\
& \quad \geq(1-\beta) E_{\Delta}\left(H_{g}(\Pi)\right)\left(1-P_{\varphi_{0} \otimes \Omega}\right) E_{\Delta}\left(H_{g}(\Pi)\right)-C g E_{\Delta}\left(H_{g}(\Pi)\right) .
\end{aligned}
$$

Proposition 5 (Virial Theorem). Let Hypotheses $(H O)-(H 1)$ be satisfied, and assume that $H_{g}(\Pi) \varphi=E \varphi$, for some $\varphi \in L^{2}\left(\mathbb{R}^{3}\right) \otimes \mathcal{F}$ with $\Gamma\left(\chi_{i}\right) \varphi=\varphi$, and for an energy $E<\Sigma_{\text {ion. }}$ Then

$$
\left\langle\varphi,\left[H_{g}(\Pi), i A\right] \varphi\right\rangle=0, \quad \text { and }\left\langle\varphi,\left[H_{g}(\Pi), i \mathrm{~d} \Gamma(a)\right] \varphi\right\rangle=0,
$$

where $\left[H_{g}(\Pi), i A\right]$ and $\left[H_{g}(\Pi), i \mathrm{~d} \Gamma(a)\right]$ are defined by (69) and (111), respectively. 
Proof. We choose $\omega \in C^{\infty}\left(\mathbb{R}^{3}\right)$ with $\omega(k)=|k|$, if $|k|>\sigma, \omega(k) \geq \sigma / 2$ for all $k$, and we define

$$
a_{\mathrm{mod}}=\frac{1}{2} \mathrm{~d} \Gamma(\nabla \omega(k) \cdot y+y \cdot \nabla \omega(k)) .
$$

As in (111) and (69), the commutators $\left[H_{\bmod }(\Pi), i \mathrm{~d} \Gamma\left(a_{\bmod }\right)\right]$ and $\left[H_{\bmod }(\Pi), i A_{\bmod }\right]$ are defined in terms of symmetric operators

$$
\begin{aligned}
{\left[H_{\mathrm{mod}}(\Pi), i \mathrm{~d} \Gamma\left(a_{\mathrm{mod}}\right)\right] } & :=\mathrm{d} \Gamma\left(|\nabla \omega|^{2}\right)-\frac{\Pi-\mathrm{d} \Gamma(k)}{M} \cdot \mathrm{d} \Gamma(\nabla \omega)-g \phi\left(i a_{\bmod } F_{x}\right), \\
{\left[H_{\bmod }(\Pi), i A_{\bmod }\right] } & :=\left[H_{\bmod }(\Pi), i \mathrm{~d} \Gamma\left(a_{\bmod }\right)\right]-\left[H_{\bmod }(\Pi), D\right] .
\end{aligned}
$$

Since $\omega(k)=|k|$ for $|k| \geq \sigma$, these operators coincide with $\left[H_{g}(\Pi), i \mathrm{~d} \Gamma(a)\right]$ and $\left[H_{g}(\Pi), i A\right]$ on states without soft bosons, that is, on the range of the projection $\Gamma\left(\chi_{i}\right)$. Therefore it is enough to prove that

$$
\left\langle\varphi,\left[H_{\mathrm{mod}}(\Pi), i \mathrm{~d} \Gamma\left(a_{\mathrm{mod}}\right)\right] \varphi\right\rangle=0
$$

and

$$
\left\langle\varphi,\left[H_{\bmod }(\Pi), i A_{\bmod }\right] \varphi\right\rangle=0 .
$$

This is done in the same way as in the proof of Lemma 40 in [FGS04].

Corollary 6. Assume Hypotheses (H0)-(H2). Fix $\beta<1$ and choose $\Sigma<\min \left(\Sigma_{\beta}\right.$, $\left.\Sigma_{i o n}\right)$, with $\Sigma_{\beta}$ and $\Sigma_{i o n}$ as in Lemma 1. Then, for sufficiently small values of $g>0$,

$$
\sigma_{p p}\left(H_{g}(\Pi)\right) \cap(-\infty, \Sigma)=\left\{E_{g}(\Pi)\right\},
$$

where $E_{g}(\Pi)$ is a simple eigenvalue, for all $\Pi$ with $E_{g}(\Pi) \leq \Sigma$.

Remark. How small $g$ has to be chosen depends on the choice of $\beta$ ( $g \ll 1-\beta$ ), on the choice of $\Sigma$ (we need (44) to hold true), on $\gamma_{j}(\Pi)\left(g \ll \inf \left\{\gamma_{j}(\Pi): j \geq 1, E_{g}(\Pi) \leq\right.\right.$ $\Sigma\}$ ), and it also depends on the distances between the eigenvalues of the atomic Hamiltonian (we must require that $g^{1 / 2} \ll \min \left\{\left|E_{j+1}^{\text {at }}-E_{j}^{\text {at }}\right|: 0 \leq j \leq n\right\}$, where $n$ is such that $E_{n}^{\text {at }} \leq \Sigma<E_{n+1}^{\text {at }}$ ).

Proof. We first prove that if $g$ is sufficiently small, then

$$
\sigma_{\mathrm{pp}}\left(H_{g}(\Pi)\left\lceil\operatorname{Ran} \Gamma\left(\chi_{i}\right)\right) \cap(-\infty, \Sigma)=\left\{E_{g}(\Pi)\right\},\right.
$$

and that $E_{g}(\Pi)$ is a simple eigenvalue of $H_{g}(\Pi)\left\lceil\operatorname{Ran} \Gamma\left(\chi_{i}\right)\right.$.

To this end, we define $\Delta_{0}=\left(-\infty,\left(E_{1}(\Pi)+E_{0}(\Pi)\right) / 2\right)$. We define intervals

$$
\Delta_{j}=\left(\frac{\left(E_{j}(\Pi)+2 E_{j-1}(\Pi)\right)}{3}, \frac{\left(E_{j}(\Pi)+E_{j+1}(\Pi)\right)}{2}\right),
$$

for $j=1, \ldots n$, where $n$ is such that $E_{n-1}(\Pi)<\Sigma \leq E_{n}(\Pi)$. Each interval $\Delta_{j}$ contains exactly one eigenvalue of the free Hamiltonian $H_{0}(\Pi)$, and $(-\infty, \Sigma) \subset \cup_{j=0}^{n} \Delta_{j}$. The absence of eigenvalues of $H_{g}(\Pi)\left\lceil\operatorname{Ran} \Gamma\left(\chi_{i}\right)\right.$ inside $\Delta_{j}$, for $j \geq 1$ and for $g$ sufficiently small, follows from Propositions 3 and 5. Next, suppose that $\psi$ is a normalized eigenvector of $H_{g}(\Pi)$ corresponding to an eigenvalue $E \in \Delta_{0}$. Without loss of generality, we can assume that $\left\langle\psi, \varphi_{0} \otimes \Omega\right\rangle$ is real; recall that $\varphi_{0}$ is the unique (up to a phase) 
normalized ground state vector of $H_{\mathrm{at}}=p^{2} / 2 m+V(x)$. Then, by Proposition 4 and Proposition 5, we have that

$$
\begin{aligned}
0 & \geq(1-\beta)\left\langle\psi,\left(1-P_{\varphi_{0} \otimes \Omega}\right) \psi\right\rangle-C g=(1-\beta)\left(1-\left\langle\psi, \varphi_{0} \otimes \Omega\right\rangle^{2}\right)-C g \\
& \geq(1-\beta)\left(1-\left\langle\psi, \varphi_{0} \otimes \Omega\right\rangle\right)-C g=\frac{1-\beta}{2}\left\|\psi-\varphi_{0} \otimes \Omega\right\|^{2}-C g .
\end{aligned}
$$

Hence

$$
\left\|\psi-\varphi_{0} \otimes \Omega\right\|^{2} \leq \frac{2 C g}{1-\beta} .
$$

If there were two orthogonal eigenvectors of $H_{g}(\Pi)\left\lceil\operatorname{Ran} \Gamma\left(\chi_{i}\right), \psi_{1}\right.$ and $\psi_{2}$, corresponding to eigenvalues in $\Delta_{0}$ then both would satisfy inequality (125), and, thus, we would conclude that

$$
\left\|\psi_{1}-\psi_{2}\right\| \leq 2 \sqrt{\frac{2 C g}{1-\beta}} .
$$

But this is impossible if $g \leq(1-\beta) / 4 C$. So, for $g$ small enough, there can only be one eigenvector of $H_{g}(\Pi)\left\lceil\operatorname{Ran} \Gamma\left(\chi_{i}\right)\right.$ corresponding to an eigenvalue in $\Delta_{0}$. In Proposition 2, we have proven that $H_{g}(\Pi)\left\lceil\operatorname{Ran} \Gamma\left(\chi_{i}\right)\right.$ has a ground state vector. This proves the fact that $E_{g}(\Pi)$ is a simple eigenvalue of $H_{g}(\Pi)\left\lceil\operatorname{Ran} \Gamma\left(\chi_{i}\right)\right.$ as well as the fact that $H_{g}(\Pi)\left\lceil\operatorname{Ran} \Gamma\left(\chi_{i}\right)\right.$ has no other eigenvalue in $\Delta_{0}$. Hence (123) follows. To complete the proof of the corollary, we need to show that

$$
\sigma_{\mathrm{pp}}\left(H_{g}(\Pi)\left\lceil\left(\operatorname{Ran} \Gamma\left(\chi_{i}\right)\right)^{\perp}\right)=\varnothing .\right.
$$

To this end, we decompose $\mathcal{F} \simeq \mathcal{F}_{i} \otimes \mathcal{F}_{s} \simeq \oplus_{n \geq 0} L_{s}^{2}\left(B_{\sigma}(0)^{\times n}, \mathrm{~d} k_{1} \ldots \mathrm{d} k_{n} ; \mathcal{F}_{i}\right)$, and we write $L^{2}\left(\mathbb{R}^{3}, \mathrm{~d} x\right) \otimes \mathcal{F} \simeq \oplus_{n \geq 0} \mathcal{H}_{n}$, where

$$
\mathcal{H}_{n}=L_{s}^{2}\left(B_{\sigma}(0)^{\times n}, \mathrm{~d} k_{1} \ldots k_{n} ; L^{2}\left(\mathbb{R}^{3}, \mathrm{~d} x\right) \otimes \mathcal{F}_{i}\right)
$$

is the space of vectors containing exactly $n$ soft, non-interacting, bosons. The Hamiltonian leaves each $\mathcal{H}_{n}$ invariant, and the restriction of $H_{g}(\Pi)$ on $\mathcal{H}_{n}$ is given by

$$
\begin{aligned}
\left(H_{g}(\Pi)\left\lceil\mathcal{H}_{n} \psi\right)\left(k_{1}, \ldots k_{n}\right)\right. & =H_{\Pi}\left(k_{1}, \ldots, k_{n}\right) \psi\left(k_{1}, \ldots k_{n}\right) \\
H_{\Pi}\left(k_{1}, \ldots, k_{n}\right) & =H_{g}\left(\Pi-k_{1}-\cdots-k_{n}\right)+\sum_{j=1}^{n}\left|k_{j}\right| .
\end{aligned}
$$

Here $H_{g}\left(\Pi-k_{1}-\cdots-k_{n}\right)$ is an operator over $L^{2}\left(\mathbb{R}^{3}, \mathrm{~d} x\right) \otimes \mathcal{F}_{i}$, the space of states with no soft bosons. We know that the only eigenvalue of $H_{g}\left(\Pi-k_{1}-\cdots-k_{n}\right)$ in $(-\infty, \Sigma)$ is its ground state energy $E_{g}\left(\Pi-k_{1}-\cdots-k_{n}\right)$ as long as $E_{g}\left(\Pi-k_{1}-\cdots-\right.$ $\left.k_{n}\right)<\Sigma$. In particular the only eigenvalue of $H_{\Pi}\left(k_{1}, \ldots, k_{n}\right)$ in $(-\infty, \Sigma)$ is given by $E_{g}\left(\Pi-k_{1}-\ldots k_{n}\right)+\left|k_{1}\right|+\cdots+\left|k_{n}\right|$ if this number is less than $\Sigma$. Thus $E \in(-\infty, \Sigma)$ is an eigenvalue of $H_{g}(\Pi)\left\lceil\mathcal{H}_{n}\right.$ if and only if there exists a set $M \subset B_{\sigma}(0)^{\times n}$ with positive measure, such that

$$
E=E_{g}\left(\Pi-k_{1}-\cdots-k_{n}\right)+\left|k_{1}\right|+\cdots+\left|k_{n}\right|
$$

for all $\left(k_{1}, \ldots, k_{n}\right) \in M$. But this is impossible because, by (44) and $\beta<1$,

$$
\left|\nabla E_{g}(\Pi)\right|=\left|\left\langle\psi_{\Pi},(\Pi-\mathrm{d} \Gamma(k)) / M \psi_{\Pi}\right\rangle\right| \leq 1
$$

for every $\Pi$ with $E_{g}(\Pi)<\Sigma$ and $g$ small enough. 


\section{Scattering Theory}

The proofs of most of the results in this section are similar to those of the corresponding results in [FGS04]. In order to give an idea of the structure of the proof of our main result (asymptotic completeness, Theorem 9), we repeat here the most important theorems, but we omit most of their proofs (we refer to the corresponding statements in [FGS04]). The main difference with respect to [FGS04] is encountered in the proof of the positivity of the asymptotic observable in Sect. 4.4: there, we propose some new ideas to control the internal degrees of freedom of the atom (which are not present in [FGS04], because there we considered free electrons coupled to the quantized radiation field).

4.1. The wave operator. The first step towards understanding scattering theory for the model studied in this paper consists in the construction of states with asymptotically free photons. This can be accomplished using asymptotic field operators, which are constructed in the next theorem. Note that in Theorems 7 and 8 we do not impose any infrared cutoff on the interaction; we can take $\sigma=0$, provided the form factor $\kappa(k)$ is smooth at $k=0$. We use the notation $L_{\omega}^{2}\left(\mathbb{R}^{3}\right)=L^{2}\left(\mathbb{R}^{3},(1+1 /|k|) \mathrm{d} k\right)$.

Theorem 7 (Existence of asymptotic field operators). Assume Hypotheses $(\mathrm{HO})-(\mathrm{HI})$ are satisfied (but $\sigma=0$ is allowed!). Fix $\beta<1$ and choose $\Sigma<\min \left(\Sigma_{\beta}, \Sigma_{\text {ion }}\right)$ (with $\Sigma_{\beta}$ as in Lemma 1). If $g \geq 0$ is so small that (44) is true, then the following results hold.

i) Let $h \in L_{\omega}^{2}\left(\mathbb{R}^{3}\right)$ and let $h_{t}(k)=e^{-i|k| t} h(k)$. Then the limit

$$
a_{+}^{\sharp}(h) \varphi=\lim _{t \rightarrow \infty} e^{i H_{g} t} a^{\sharp}\left(h_{t}\right) e^{-i H_{g} t} \varphi
$$

exists for all $\varphi \in \operatorname{Ran} E_{\Sigma}\left(H_{g}\right)$.

ii) Let $h, g \in L_{\omega}^{2}\left(\mathbb{R}^{3}\right)$. Then

$$
\left[a_{+}(g), a_{+}^{*}(h)\right]=(g, h) \text { and }\left[a_{+}^{\sharp}(g), a_{+}^{\sharp}(h)\right]=0,
$$

in the sense of quadratic forms on $\operatorname{Ran} E_{\Sigma}\left(H_{g}\right)\left(a^{\sharp}(h)\right.$ means either $a^{*}(h)$ or $\left.a(h)\right)$.

iii) Let $h \in L_{\omega}^{2}\left(\mathbb{R}^{3}\right)$, and let $M:=\sup \{|k|: h(k) \neq 0\}$ and $m:=\inf \{|k|: h(k) \neq 0\}$. Then

$$
\begin{aligned}
& a_{+}^{*}(h) \operatorname{Ran} \chi\left(H_{g} \leq E\right) \subset \operatorname{Ran} \chi\left(H_{g} \leq E+M\right), \\
& a_{+}(h) \operatorname{Ran} \chi\left(H_{g} \leq E\right) \subset \operatorname{Ran} \chi\left(H_{g} \leq E-m\right),
\end{aligned}
$$

if $E \leq \Sigma$.

iv) Let $h_{i} \in L_{\omega}^{2}\left(\mathbb{R}^{3}\right)$ for $i=1, \ldots n$. Put $M_{i}=\sup \left\{|k|: h_{i}(k) \neq 0\right\}$ and assume $\varphi \in \operatorname{Ran} E_{\lambda}\left(H_{g}\right)$. Then if $\lambda+\sum_{i=1}^{n} M_{i} \leq \Sigma$ we have that $\varphi \in D\left(a_{+}^{\sharp}\left(h_{1}\right) \ldots a_{+}^{\sharp}\left(h_{n}\right)\right)$, the limits

$$
a_{+}^{\sharp}\left(h_{1}\right) \ldots a_{+}^{\sharp}\left(h_{n}\right) \varphi=\lim _{t \rightarrow \infty} e^{i H_{g} t} a^{\sharp}\left(h_{1, t}\right) \ldots a^{\sharp}\left(h_{n, t}\right) e^{-i H_{g} t} \varphi
$$

exist, and

$$
\left\|a_{+}^{\sharp}\left(h_{1}\right) \ldots a_{+}^{\sharp}\left(h_{n}\right)\left(H_{g}+i\right)^{-n / 2}\right\| \leq C\left\|h_{1}\right\|_{\omega} \ldots\left\|h_{n}\right\|_{\omega} .
$$


v) If $\varphi \in E_{\Sigma}\left(H_{g}\right) \mathcal{H}_{\text {das }}$ and $h \in L_{\omega}^{2}\left(\mathbb{R}^{3}\right)$,

$$
a_{+}(h) \varphi=0 .
$$

(Wave packets of dressed atom states are vacua of the asymptotic field operators.)

The proof of this theorem is very similar to the one of Theorem 13 and Lemma 14 in [FGS04]. It relies on a propagation estimate for the center of mass of the atom (see Proposition 12 in [FGS04]), which guarantees that if the energy is smaller than $\Sigma_{\beta}$, then the asymptotic velocity of the atom is bounded above by $\beta$ (here $\beta<1$ ), and it exploits the fact that, because the energy is below the ionization threshold, the electron is exponentially bound to the nucleus. These two facts and the fact that the propagation speed of photons is the speed of light are sufficient to prove that the interaction between the atom and asymptotically freely propagating photons tends to zero, as $t \rightarrow \infty$.

The existence of asymptotic field operators allows us to introduce the wave operator $\Omega_{+}$of the system. In order to define $\Omega_{+}$, we add a new copy of the Fock space $\mathcal{F}$ describing states of free photons to the physical Hilbert space $\mathcal{H}=L^{2}\left(\mathbb{R}^{3}, \mathrm{~d} X\right) \otimes L^{2}\left(\mathbb{R}^{3}, \mathrm{~d} x\right) \otimes \mathcal{F}$. We define the extended Hamiltonian

$$
\tilde{H}_{g}=H_{g} \otimes 1+1 \otimes \mathrm{d} \Gamma(|k|)
$$

on the extended Hilbert space $\widetilde{\mathcal{H}}=\mathcal{H} \otimes \mathcal{F}$. In the next theorem, we establish the existence of the wave operator $\Omega_{+}$as an isometry from a subspace of $\widetilde{\mathcal{H}}$ to a subspace of the physical Hilbert space $\mathcal{H}$. The "scattering identification map", $I$, used in the definition of the wave operator $\Omega_{+}$, is defined in Appendix A.6.

Theorem 8 (Existence of the wave operator). Let Hypotheses $(\mathrm{HO})-(\mathrm{Hl})$ be satisfied (but $\sigma=0$ is allowed). Fix $\beta<1$, and choose $\Sigma<\min \left(\Sigma_{\beta}, \Sigma_{i o n}\right)$ (with $\Sigma_{\beta}, \Sigma_{i o n}$ defined as in Lemma 1). Then if $g \geq 0$ is small enough (depending on $\beta$ and $\Sigma$ ) the limit

$$
\Omega_{+} \varphi:=\lim _{t \rightarrow \infty} e^{i H_{g} t} I e^{-i \tilde{H}_{g} t}\left(P_{\text {das }} \otimes 1\right) \varphi
$$

exists for an arbitrary vector $\varphi$ in the dense subspace of $\operatorname{Ran} E_{\Sigma}(\tilde{H})$ spanned by finite linear combinations of vectors of the form $\gamma \otimes a^{*}\left(h_{1}\right) \ldots a^{*}\left(h_{n}\right) \Omega$, where $\gamma=E_{\lambda}\left(H_{g}\right) \gamma$, $h_{i} \in L_{\omega}^{2}\left(\mathbb{R}^{3}\right)$, and with $\lambda+\sum_{i} \sup \left\{|k|: h_{i}(k) \neq 0\right\} \leq \Sigma$. If $\varphi=\gamma \otimes a^{*}\left(h_{1}\right) \ldots a^{*}\left(h_{n}\right) \Omega$ belongs to this space then

$$
\Omega_{+} \varphi=a_{+}^{*}\left(h_{1}\right) \ldots a_{+}^{*}\left(h_{n}\right) P_{\text {das }} \gamma .
$$

Furthermore $\left\|\Omega_{+}\right\|=1$, and $\Omega_{+}$has therefore a unique extension, also denoted by $\Omega_{+}$, to $E_{\Sigma}\left(\tilde{H}_{g}\right) \tilde{\mathcal{H}}$. On $\left(P_{\text {das }} \otimes 1\right) E_{\Sigma}\left(\tilde{H}_{g}\right) \tilde{\mathcal{H}}$, the operator $\Omega_{+}$is isometric. For all $t \in \mathbb{R}$,

$$
e^{-i H_{g} t} \Omega_{+}=\Omega_{+} e^{-i \tilde{H}_{g} t}
$$

For the proof of this theorem we refer to the proof of Theorem 15 in [FGS04], which is almost identical. From Eq. (132) we see that vectors in the range of $\Omega_{+}$are limits of linear combination of vectors describing the wave packet of dressed atom states and configurations of finitely many asymptotically freely moving photons. Physically, it is expected that the asymptotic evolution of every state with an energy below the ionization threshold of the atom (that is $\Sigma<\Sigma_{\text {ion }}$ ) and so small that the atom does not propagate 
with a velocity larger than one (i.e., $\Sigma<\Sigma_{\beta=1}$ ) can be approximated by linear combinations of vectors describing a dressed atom state and a configuration of finitely many freely propagating photons. More precisely, one expects that

$$
\operatorname{Ran} \Omega_{+} \supset \operatorname{Ran} E_{\Sigma}\left(H_{g}\right), \quad \text { if } \Sigma<\min \left(\Sigma_{\beta=1}, \Sigma_{\text {ion }}\right) .
$$

This statement is called asymptotic completeness of Rayleigh scattering. Due to technical difficulties, we can only prove asymptotic completeness for states with energy less than a threshold energy $\Sigma<\min \left(\Sigma_{\beta=1 / 3}, \Sigma_{\exp }\right)$ and assuming that the coupling constant $g$ is small enough. The following theorem is our main result.

Theorem 9 (Asymptotic Completeness). Assume that Hypotheses $(\mathrm{HO})-(\mathrm{H} 2)$ are satisfied (see Eqs. (27), and (63)). Fix $\beta<1 / 3$, and choose $\Sigma<\min \left(\Sigma_{\beta}, \Sigma_{\text {ion }}\right)$ (with $\Sigma_{\beta}$ and $\Sigma_{i o n}$ as in Lemma 1). Then, for $g>0$ sufficiently small,

$$
\operatorname{Ran} \Omega_{+} \supset E_{\Sigma}\left(H_{g}\right) \mathcal{H}
$$

Remark. The allowed range of values of $g$ depends on the value of $(1 / 3-\beta)$; (we need that $g \ll 1 / 3-\beta)$, on the choice of $\Sigma$ ( $g$ must be small enough in order for Eq. (44) to hold true), on the value of $\alpha=\inf \left\{\gamma_{j}(\Pi): j \geq 1, E_{g}(\Pi)<\Sigma\right\}(g \ll \alpha)$, and on $\delta=\min \left\{\left|E_{j+1}^{\text {at }}-E_{j}^{\text {at }}\right|: 0 \leq j \leq n\right\}$, with $n$ such that $E_{n}^{\text {at }} \leq \Sigma<E_{n+1}^{\text {at }}\left(g^{1 / 2} \ll \delta\right)$. As remarked in Sect. 2, the assumption that $g$ is positive is not necessary, it only simplifies the notation (but $g=0$ is not allowed, because in this case the fiber Hamiltonian $H_{g}(\Pi)$ has embedded eigenvalues).

Theorem 9 will be seen to follow from Lemma 11, where we show that it suffices to prove an analogous statement for a modified Hamiltonian $H_{\text {mod }}$ (introduced in the next section) and from Theorem 19 in Sect. 4.4, where asymptotic completeness for $H_{\text {mod }}$ is proven.

4.2. The modified Hamiltonian. The fact that the bosons are massless leads to some technical difficulties connected with the unboundedness of the operator $N=\mathrm{d} \Gamma$ (1) with respect to the Hamiltonian. However, as long as the infrared cutoff is strictly positive, the number of bosons with energy below $\sigma$ is conserved. This allows us to introduce a modified Hamiltonian, where the dispersion law of the soft, non-interacting, photons is changed. We define

$$
H_{\mathrm{mod}}=\frac{P^{2}}{2 M}+\frac{p^{2}}{2 m}+V(x)+\mathrm{d} \Gamma(\omega)+g \phi\left(G_{X, x}\right),
$$

and we assume that the dispersion law $\omega$ has the following properties:

Hypothesis (H3). $\omega \in \mathcal{C}^{\infty}\left(\mathbb{R}^{3}\right)$, with $\omega(k) \geq|k|$, $\omega(k)=|k|$, for $|k| \geq \sigma$, $\omega(k) \geq \sigma / 2$, for all $k \in \mathbb{R}^{3}$, $\sup _{k}|\nabla \omega(k)| \leq 1$, and $\nabla \omega(k) \neq 0$ unless $k=0$. Furthermore, $\omega\left(k_{1}+k_{2}\right) \leq \omega\left(k_{1}\right)+\omega\left(k_{2}\right)$ for all $k_{1}, k_{2} \in \mathbb{R}^{3}$. (Here $\sigma>0$ is the infrared cutoff defined in Hypothesis (H1).)

The two Hamiltonians, $H_{g}$ and $H_{\text {mod }}$, agree on states of the system without soft bosons. Recall that $\chi_{i}(k)$ is the characteristic function of the set $\{k:|k| \geq \sigma\}$ and that the operator $\Gamma\left(\chi_{i}\right)$ is the orthogonal projection onto the subspace of vectors describing states without soft bosons. It is straightforward to check that $H_{g}$ and $H_{\text {mod }}$ leave the range of the projection $\Gamma\left(\chi_{i}\right)$ invariant and that

$$
H_{g}\left\lceil\operatorname{Ran} \Gamma\left(\chi_{i}\right)=H_{\bmod }\left\lceil\operatorname{Ran} \Gamma\left(\chi_{i}\right) .\right.\right.
$$


The same conclusion can be reached using the unitary operator $U: \mathcal{H} \rightarrow \mathcal{H}_{i} \otimes \mathcal{F}_{S}$ introduced in Sect. 2. On the factorized Hilbert space $\mathcal{H}_{i} \otimes \mathcal{F}_{s}$, the Hamiltonians $H_{g}$ and $H_{\text {mod }}$ are given by

$$
\begin{aligned}
U H_{g} U^{*} & =H_{i} \otimes 1+1 \otimes \mathrm{d} \Gamma(|k|), \\
U H_{\mathrm{mod}} U^{*} & =H_{i} \otimes 1+1 \otimes \mathrm{d} \Gamma(\omega) \text { with } \\
H_{i} & =\frac{P^{2}}{2 M}+\frac{p^{2}}{2 m}+V(x)+\mathrm{d} \Gamma(|k|)+g \phi\left(G_{X, x}\right),
\end{aligned}
$$

and we see explicitly that the two Hamiltonians agree on states without soft bosons.

The modified Hamiltonian $H_{\text {mod }}$, just like the physical Hamiltonian $H_{g}$, commutes with spatial translations, i.e., $\left[H_{\bmod }, \Pi\right]=0$, where $\Pi=P+\mathrm{d} \Gamma(k)$ is the total momentum of the system. In the representation of the system on the Hilbert space $L^{2}\left(\mathbb{R}_{\Pi}^{3} ; L^{2}\left(\mathbb{R}^{3}, \mathrm{~d} x\right) \otimes \mathcal{F}\right)$, the modified Hamiltonian $H_{\text {mod }}$ is given by

$$
\begin{aligned}
\left(T H_{\mathrm{mod}} T^{*} \psi\right)(\Pi) & =H_{\bmod }(\Pi) \psi(\Pi), \\
H_{\bmod }(\Pi) & =\frac{(\Pi-\mathrm{d} \Gamma(k))^{2}}{2 M}+\frac{p^{2}}{2 m}+V(x)+\mathrm{d} \Gamma(\omega)+g \phi\left(F_{x}\right),
\end{aligned}
$$

where $T: \mathcal{H} \rightarrow L^{2}\left(\mathbb{R}^{3}, d \Pi ; L^{2}\left(\mathbb{R}^{3}, \mathrm{~d} x\right) \otimes \mathcal{F}\right)$ has been defined in Sect. 2 .

The fiber Hamiltonians $H_{g}(\Pi)$ and $H_{\bmod }(\Pi)$ commute with the projection $\Gamma\left(\chi_{i}\right)$ and agree on its range,

$$
H_{g}(\Pi)\left\lceil\operatorname{Ran} \Gamma\left(\chi_{i}\right)=H_{\bmod }(\Pi)\left\lceil\operatorname{Ran} \Gamma\left(\chi_{i}\right) .\right.\right.
$$

In the proof of Proposition 2 we have shown that if $\beta<1$ and $\Sigma<\min \left(\Sigma_{\beta}, \Sigma_{\text {ion }}\right)$ then, for $g$ small enough,

$$
\inf \sigma\left(H_{\text {mod }}(\Pi)\right)=\inf \sigma\left(H_{g}(\Pi)\right)=E_{g}(\Pi),
$$

where $E_{g}(\Pi)$ is a simple eigenvalue of $H_{g}(\Pi)$ and of $H_{\bmod }(\Pi)$, as long as $E_{g}(\Pi) \leq \Sigma$. Moreover, the corresponding dressed atom states coincide. Since the subspace $\mathcal{H}_{\text {das }}$ is defined in terms of the dressed atom states $\psi_{\Pi}$, it follows that vectors in $\mathcal{H}_{\text {das }}$ also describe dressed atom wave packets for the dynamics generated by the modified Hamiltonian $H_{\text {mod }}$.

We remark that

$$
\sigma_{\mathrm{pp}}\left(H_{\mathrm{mod}}(\Pi)\right) \cap(-\infty, \Sigma)=\left\{E_{g}(\Pi)\right\},
$$

for all $\Pi \in \mathbb{R}^{3}$ with $E_{g}(\Pi) \leq \Sigma$, and for $g$ sufficiently small; see Eq. (123) and Corollary 6.

Next, we discuss the scattering theory for the modified Hamiltonian $H_{\text {mod }}$. As in Theorem 8 we fix $\beta<1$ and we choose $\Sigma<\min \left(\Sigma_{\beta}, \Sigma_{\text {ion }}\right)$. Then, by the assumption that $\omega(k)=|k|$ for $|k| \geq \sigma$, and since $\mathrm{d} \Gamma(|k|-\omega)$ commutes with $H_{g}$ and $H_{\text {mod }}$ we have that

$$
\begin{aligned}
e^{i H_{\bmod } t} a^{\sharp}\left(e^{-i \omega t} h\right) e^{-i H_{\bmod } t} & =e^{i H_{g} t} e^{-i \mathrm{~d} \Gamma(|k|-\omega) t} a^{\sharp}\left(e^{-i \omega t} h\right) e^{i \mathrm{~d} \Gamma(|k|-\omega) t} e^{-i H_{g} t} \\
& =e^{i H_{g} t} a^{\sharp}\left(e^{-i|k| t} h\right) e^{-i H_{g} t},
\end{aligned}
$$

for all $t$. It follows that the limit

$$
a_{\mathrm{mod},+}^{\sharp}(h) \varphi=\lim _{t \rightarrow \infty} e^{i H_{\bmod } t} a^{\sharp}\left(e^{-i \omega t} h\right) e^{-i H_{\bmod } t} \varphi
$$


exists and that $a_{\text {mod, }+}^{\sharp}(h) \varphi=a_{+}^{\sharp}(h) \varphi$, for all $\varphi \in \operatorname{Ran} E_{\Sigma}\left(H_{\text {mod }}\right) \subset \operatorname{Ran} E_{\Sigma}\left(H_{g}\right)$ and for all $h \in L_{\omega}^{2}\left(\mathbb{R}^{3}\right)$. This and the fact that vectors in $\mathcal{H}_{\text {das }}$ describe dressed atom states for $H_{g}$ and for $H_{\text {mod }}$ show that the asymptotic states constructed with the help of the Hamiltonians $H_{g}$ and $H_{\text {mod }}$ coincide.

On the extended Hilbert space $\tilde{\mathcal{H}}=\mathcal{H} \otimes \mathcal{F}$, we define the extended modified Hamiltonian

$$
\tilde{H}_{\text {mod }}=H_{\text {mod }} \otimes 1+1 \otimes \mathrm{d} \Gamma(\omega) \text {. }
$$

In terms of $H_{\text {mod }}$ and $\tilde{H}_{\text {mod }}$ we also define a modified version, $\tilde{\Omega}_{+}^{\text {mod }}$, of the wave operator $\Omega_{+}$introduced in Sect. 4.1.

Lemma 10. Let Hypotheses (HO), (H1) and (H3) be satisfied $(\sigma=0$ in Hypothesis (HI) is allowed, and then $\left.H_{\text {mod }}=H_{g}\right)$. Fix $\beta<1$ and $\Sigma<\min \left(\Sigma_{\beta}, 0\right)$. Then if $g \geq 0$ is sufficiently small, depending on $\beta$ and $\Sigma$, the limit

$$
\tilde{\Omega}_{+}^{\text {mod }} \varphi=\lim _{t \rightarrow \infty} e^{i H_{\text {mod }} t} I e^{-i \tilde{H}_{\text {mod }} t} \varphi
$$

exists for all $\varphi \in E_{\Sigma}\left(\tilde{H}_{\text {mod }}\right) \tilde{\mathcal{H}}$. Moreover, the modified wave operator $\Omega_{+}^{\text {mod }}$, defined by $\Omega_{+}^{\text {mod }}=\tilde{\Omega}_{+}^{\text {mod }}\left(P_{\text {das }} \otimes 1\right)$, agrees with $\Omega_{+}$on $\operatorname{Ran} E_{\Sigma}\left(\tilde{H}_{\text {mod }}\right)$. That is,

$$
\Omega_{+}^{\bmod } \varphi=\Omega_{+} \varphi
$$

for all $\varphi \in \operatorname{Ran} E_{\Sigma}\left(\tilde{H}_{m o d}\right) \subset \operatorname{Ran} E_{\Sigma}\left(\tilde{H}_{g}\right)$.

We now extend the domain of $\Omega_{+}$to include arbitrarily many soft, non-interacting bosons. As a byproduct we obtain a proof of (138). To start with, we recall the isomorphism $U: \mathcal{F} \rightarrow \mathcal{F}_{i} \otimes \mathcal{F}_{S}$ introduced in Sec. 2 and define a unitary isomorphism $U \otimes U: \tilde{\mathcal{H}} \rightarrow \mathcal{H}_{i} \otimes \mathcal{F}_{i} \otimes \mathcal{F}_{s} \otimes \mathcal{F}_{s}$ separating interacting from soft bosons in the extended Hilbert space $\tilde{\mathcal{H}}$. With respect to this factorization the extended Hamiltonian $\tilde{H}$ becomes $\tilde{H}_{g}=\tilde{H}_{i} \otimes 1 \otimes 1+1 \otimes 1 \otimes \mathrm{d} \Gamma(|k|) \otimes 1+1 \otimes 1 \otimes 1 \otimes \mathrm{d} \Gamma(|k|)$, where $\tilde{H}_{i}=H_{i} \otimes 1+1 \otimes \mathrm{d} \Gamma(|k|)$. As an operator from $\mathcal{H}_{i} \otimes \mathcal{F}_{i} \otimes \mathcal{F}_{s} \otimes \mathcal{F}_{s}$ to $\mathcal{H}_{i} \otimes \mathcal{F}_{s}$, the wave operator $\Omega_{+}$acts as

$$
U \Omega_{+}\left(U^{*} \otimes U^{*}\right)=\Omega_{+}^{\text {int }} \otimes \Omega_{+}^{\text {soft }}
$$

where $\Omega_{+}^{\text {int }}: \mathcal{H}_{i} \otimes \mathcal{F}_{i} \rightarrow \mathcal{H}_{i}$ is given by

$$
\Omega_{+}^{\text {int }}=s-\lim _{t \rightarrow \infty} e^{i H_{i} t} I e^{-i \tilde{H}_{i} t}\left(P_{\mathrm{das}}^{\text {int }} \otimes 1\right)
$$

while $\Omega_{+}^{\text {soft }}: \mathcal{F}_{S} \otimes \mathcal{F}_{S} \rightarrow \mathcal{F}_{s}$ is given by

$$
\Omega_{+}^{\text {soft }}=I\left(P_{\Omega} \otimes 1\right),
$$

where $P_{\Omega}$ is the orthogonal projection onto the vacuum vector $\Omega \in \mathcal{F}_{s}$. In view of (139) and (140), the domain of $\Omega_{+}$can obviously be extended to $\operatorname{Ran} E_{\Sigma}\left(\tilde{H}_{i}\right) \otimes \mathcal{F}_{s} \otimes$ $\mathcal{F}_{s} \supset \operatorname{Ran} E_{\Sigma}\left(\tilde{H}_{g}\right)$. For the modified wave operator $\Omega_{+}^{\bmod }=\tilde{\Omega}_{+}^{\bmod }\left(P_{\text {das }} \otimes 1\right)$, we have $\Omega_{+}^{\text {mod }}=\Omega_{+, \text {int }}^{\text {int }} \otimes \Omega_{+}^{\text {soft }}$, and from $H_{g}\left\lceil\operatorname{Ran} \Gamma\left(\chi_{i}\right)=H_{\text {mod }}\left\lceil\operatorname{Ran} \Gamma\left(\chi_{i}\right)\right.\right.$ it follows that $\Omega_{+, \text {int }}^{\text {int }}=\Omega_{+}^{\text {int }}$. Consequently, also $\Omega_{+}^{\text {mod }}$ is well defined on $\operatorname{Ran} E_{\Sigma}\left(\tilde{H}_{i}\right) \otimes \mathcal{F}_{s} \otimes \mathcal{F}_{s}$ and $\Omega_{+}^{\bmod }=\Omega_{+}$.

We summarize the main conclusions in a lemma. 
Lemma 11. Let the assumptions of Lemma 10 be satisfied, and let $\Omega_{+}$be defined on $\operatorname{Ran} E_{\Sigma} \otimes \mathcal{F}_{S} \otimes \mathcal{F}_{s}$, as explained above. Then

$$
\operatorname{Ran} \Omega_{+} \cong \operatorname{Ran} \Omega_{+}^{i n t} \otimes \mathcal{F}_{S}
$$

in the factorization $\mathcal{H} \cong \mathcal{H}_{i} \otimes \mathcal{F}_{s}$. In particular, the following statements are equivalent:

i) $\operatorname{Ran} \Omega_{+} \supset E_{\Sigma}\left(H_{g}\right) \mathcal{H}$,

ii) $\operatorname{Ran} \Omega_{+} \supset \Gamma\left(\chi_{i}\right) E_{\Sigma}\left(H_{g}\right) \mathcal{H}$,

iii) $\operatorname{Ran} \Omega_{+} \supset E_{\Sigma}\left(H_{\text {mod }}\right) \mathcal{H}$,

iv) $\operatorname{Ran} \Omega_{+} \supset \Gamma\left(\chi_{i}\right) E_{\Sigma}\left(H_{\text {mod }}\right) \mathcal{H}$.

4.3. Existence of the asymptotic observable and of the inverse wave operator. Fix $\beta<1$ and choose $\Sigma<\min \left(\Sigma_{\beta}, \Sigma_{\text {ion }}\right)$ (recall from Lemma 1 that $\left.\Sigma_{\beta}=E_{0}^{\text {at }}+M \beta^{2} / 2\right)$. We choose numbers $\beta_{1}, \beta_{2}, \beta_{3}$ and $\gamma$ such that

$$
\beta<\beta_{1}<\beta_{2}<\beta_{3}<\gamma .
$$

Definition. We pick a function $\chi_{\gamma} \in \mathbb{C}^{\infty}(\mathbb{R} ;[0,1])$ such that $\chi_{\gamma} \equiv 1$ on $[\gamma, \infty)$ and $\chi_{\gamma} \equiv 0$ on $\left(-\infty, \beta_{3}\right]$. Our asymptotic observable $W$ is defined by

$$
W=s-\lim _{t \rightarrow \infty} e^{i H_{\text {mod }} t} f\left(H_{\text {mod }}\right) \mathrm{d} \Gamma\left(\chi_{\gamma}(|y| / t)\right) f\left(H_{\text {mod }}\right) e^{-i H_{\text {mod }} t},
$$

where the energy cutoff $f$ is smooth and supported in $(-\infty, \Sigma)$. For the existence of this limit, see Proposition 13 below.

The physical meaning of the asymptotic observable is easy to understand: $W$ measures the number of photons that are propagating with an asymptotic velocity larger than $\gamma$. We will prove in Sect. 4.4 that $W$ is positive when restricted to the subspace of vectors orthogonal to the space $\mathcal{H}_{\text {das }}$ of wave packets of dressed atom states. Instead of inverting the wave operator $\Omega_{+}$directly, we can then invert it with respect to the asymptotic observable $W$. More precisely, we define an operator $W_{+}: \mathcal{H} \rightarrow \tilde{\mathcal{H}}=\mathcal{H} \otimes \mathcal{F}$, called the inverse wave operator, such that $W=\widetilde{\Omega}_{+} W_{+}$. Then, using the positivity of $W$, we can construct an inverse of $\widetilde{\Omega}_{+}$. In order to define $W_{+}$, we need to split each boson state into two parts, the second part being mapped to the second Fock-space of prospective asymptotically freely moving bosons.

Definition. We define $j_{t}: \mathfrak{h}=L^{2}\left(\mathbb{R}^{3}, d k\right) \rightarrow \mathfrak{h} \oplus \mathfrak{h}$ as follows: let $j_{t} h=\left(j_{0, t} h, j_{\infty}, t h\right)$, where $j_{\sharp, t}(y)=j_{\sharp}(|y| / t), j_{\sharp} \in C^{\infty}(\mathbb{R} ;[0,1]), j_{0}+j_{\infty} \equiv 1, j_{0} \equiv 1$ on $\left(-\infty, \beta_{2}\right]$, $\operatorname{supp}\left(j_{0}\right) \subset\left(-\infty, \beta_{3}\right]$ while $j_{\infty} \equiv 1$ on $\left[\beta_{3}, \infty\right)$ and $\operatorname{supp}\left(j_{\infty}\right) \subset\left[\beta_{2}, \infty\right)$. Then the inverse wave operator $W_{+}$is given by

$$
W_{+}=s-\lim _{t \rightarrow \infty} e^{i \tilde{H}_{\text {mod }} t} f\left(\tilde{H}_{\text {mod }}\right) \breve{\Gamma}\left(j_{t}\right) \mathrm{d} \Gamma\left(\chi_{\gamma}(|y| / t)\right) f\left(H_{\text {mod }}\right) e^{-i H_{\text {mod }} t},
$$

where $f$ is a smooth energy cutoff supported in $(-\infty, \Sigma)$. See Appendix A.5 for the definition of the operator $\breve{\Gamma}\left(j_{t}\right)$. For the existence of this limit, see Proposition 14.

Note that, since by definition $\beta<\gamma$, the photons which propagate with velocity larger than $\gamma$ are asymptotically free. To prove this fact, notice first that Lemma 1 continues to hold with $H_{g}$ replaced by $H_{\text {mod }}$. Hence, the assumption that supp $f \subset(-\infty, \Sigma)$ (where $f$ is the energy cutoff appearing in the definition of $W$ and $\left.W_{+}\right)$with $\Sigma<\min \left(\Sigma_{\beta}, \Sigma_{\text {ion }}\right)$ 
guarantees, for sufficiently small $g$, that both the nucleus and the electron remain inside a ball of radius $\beta t$ around the origin. In fact, using the assumption $\Sigma<\Sigma_{\beta}$ (and $g$ small enough), we can prove, analogously to Proposition 12 in [FGS04], that

$$
s-\lim _{t \rightarrow \infty} h(|X| / t) f\left(H_{\mathrm{mod}}\right) e^{-i t H_{\mathrm{mod}}}=0
$$

for any $h \in C^{\infty}(\mathbb{R})$ with $h^{\prime} \in C_{0}^{\infty}(\mathbb{R})$, supp $h \subset(\beta, \infty)$ and for any $f \in C_{0}^{\infty}(\mathbb{R})$ with supp $f \subset(-\infty, \Sigma)$. Recall that $X$ is the coordinate of the center of mass of the atom. Moreover, the assumption that $\Sigma<\Sigma_{\text {ion }}$ implies that the electron and the nucleus remain exponentially bound for all times; therefore, both the electron and the nucleus are localized inside the ball of radius $\beta t$. As a consequence, the interaction strength between the nucleus (or the electron) and those bosons counted by $\mathrm{d} \Gamma\left(\chi_{\gamma}(|y| / t)\right)$ decays in $t$ at an integrable rate. To establish this fact rigorously we need the following lemma, similar to Lemma 9 in [FGS04].

Lemma 12. Assume that Hypothesis (H1) is satisfied and that $R^{\prime}>R>0$. Then, for every $\mu \geq 0$, there exists a constant $C_{\mu}$ such that

$$
\sup _{X, x \in \mathbb{R}^{3}} e^{-\alpha|x|} \chi(|X| \leq R)\left\|\chi\left(|y| \geq R^{\prime}\right) G_{X, x}\right\| \leq C_{\mu}\left(R^{\prime}-R\right)^{-\mu} .
$$

Moreover, if $\Sigma<\Sigma_{\text {ion }}$, we have

$$
\left\|\phi\left(\chi\left(|y| \geq R^{\prime}\right) G_{X, x}\right) \chi(|X| \leq R) E_{\Sigma}\left(H_{m o d}\right)\right\| \leq C_{\mu}\left(R^{\prime}-R\right)^{-\mu} .
$$

Remark. In the proof of the existence of the operators $W$ and $W_{+}$, where we use this lemma, typically $R=\beta t$ and $R^{\prime}=\gamma t$. Hence the r.h.s. of (145) gives a decay in time which is integrable if we choose $\mu$ large enough.

Proof. To prove (144), we first choose $\varepsilon=\left(R^{\prime}-R\right) / 2 \lambda>0$, with $\lambda=\max \left(\lambda_{n}, \lambda_{e}\right)$ (recall that $\lambda_{e}=m_{n} / M$ and $\lambda_{n}=m_{e} / M$ ) and we observe that

$$
\begin{aligned}
e^{-\alpha|x|} \chi(|X| \leq R)\left\|\chi\left(|y| \geq R^{\prime}\right) G_{X, x}\right\| \leq & \chi(|x| \leq \varepsilon) \chi(|X| \leq R) \\
& \times\left\|\chi\left(|y| \geq R^{\prime}\right) G_{X, x}\right\|+e^{-\alpha \varepsilon}\left\|G_{X, x}\right\| .
\end{aligned}
$$

Using that $G_{X, x}(k)=e^{-i\left(X+\lambda_{e} x\right) \cdot k} \kappa_{e}(k)+e^{-i\left(X-\lambda_{n} x\right) \cdot k} \kappa_{n}(k)$, it follows that

$$
\left\|G_{X, x}\right\|^{2} \leq 2 \int \mathrm{d} k\left(\left|\kappa_{e}(k)\right|^{2}+\left|\kappa_{n}(k)\right|^{2}\right) .
$$

Hence the second term on the r.h.s. of (146) can be bounded by $C \varepsilon^{-\mu}=C\left(R^{\prime}-R\right)^{-\mu}$ (because $\varepsilon^{\mu} e^{-\alpha \varepsilon}$ is bounded). Moreover the square of the first term on the r.h.s. of (146) can be estimated by

$$
\begin{aligned}
\chi(|x| \leq \varepsilon) \chi(|X| \leq R)\left\|\chi\left(|y| \geq R^{\prime}\right) G_{X, x}\right\|^{2} \\
\leq 2 \chi(|x| \leq \varepsilon) \chi(|X| \leq R) \int \mathrm{d} y \chi\left(|y| \geq R^{\prime}\right) \\
\quad \times\left(\left|\hat{\kappa}_{e}\left(X+\lambda_{e} x-y\right)\right|^{2}+\left|\hat{\kappa}_{n}\left(X-\lambda_{n} x-y\right)\right|^{2}\right) \\
\leq 2 \int \mathrm{d} y \chi\left(\left|X+\lambda_{e} x-y\right| \geq \frac{R^{\prime}-R}{2}\right)\left|\hat{\kappa}_{e}\left(X+\lambda_{e} x-y\right)\right|^{2} \\
\quad+2 \int \mathrm{d} y \chi\left(\left|X-\lambda_{n} x-y\right| \geq \frac{R^{\prime}-R}{2}\right)\left|\hat{\kappa}_{n}\left(X-\lambda_{n} x-y\right)\right|^{2} \\
\leq C \int_{|y| \geq \frac{R^{\prime}-R}{2}} \mathrm{~d} y\left(\left|\hat{\kappa}_{n}(y)\right|^{2}+\left|\hat{\kappa}_{e}(y)\right|^{2}\right)
\end{aligned}
$$


for all $X$ and $x$. Here we used that, from $|y| \geq R^{\prime},|X| \leq R$, and since, by definition of $\varepsilon$, $\lambda_{e}|x| \leq \lambda_{e} \varepsilon \leq\left(R^{\prime}-R\right) / 2$ and $\lambda_{n}|x| \leq \lambda_{n} \varepsilon \leq\left(R^{\prime}-R\right) / 2$, we have that $\left|X+\lambda_{e} x-y\right| \geq$ $|y|-|X|-\lambda_{e}|x| \geq\left(R^{\prime}-R\right) / 2$ and analogously $\left|X-\lambda_{n}-y\right| \geq\left(R^{\prime}-R\right) / 2$. Since $\kappa_{e}, \kappa_{n} \in C_{0}^{\infty}\left(\mathbb{R}^{3}\right)$, their Fourier transforms decay faster than any power, and hence (148) implies (144).

To prove (145), we use that

$$
\begin{aligned}
& \left\|\phi\left(\chi\left(|y| \geq R^{\prime}\right) G_{X, x}\right) \chi(|X| \leq R) E_{\Sigma}\left(H_{\text {mod }}\right)\right\| \\
& \leq\left\|e^{-\alpha|x|} \chi(|X| \leq R) \phi\left(\chi\left(|y| \geq R^{\prime}\right) G_{X, x}\right)(N+1)^{-1}\right\| \\
& \quad\left\|(N+1) e^{\alpha|x|} E_{\Sigma}\left(H_{\text {mod }}\right)\right\| \\
& \leq C \sup _{x, X} e^{-\alpha|x|} \chi(|X| \leq R)\left\|\chi\left(|y| \geq R^{\prime}\right) G_{X, x}\right\|
\end{aligned}
$$

because $\left\|(N+1) e^{\alpha|x|} E_{\Sigma}\left(H_{\text {mod }}\right)\right\|$ is finite (because $\Sigma<\Sigma_{\text {ion }}$ and by a simple commutation). Equation (145) then follows from (144).

The decay of the interaction determined in the last lemma is one of the two key ingredients for proving the existence of $W$ and $W_{+}$. The other one is a propagation estimate for the photons, analogous to Proposition 24 in [FGS04], but with the cutoff for $x / t$ (in [FGS04], $x$ is the position of the electron) replaced by a cutoff for the asymptotic velocity $X / t$ of the center of mass of the nucleus-electron compound (the reason why we can introduce here a cutoff in $X / t$ is that, because of (143), we know it can not exceed $\beta$ ).

For the details of the proof of the next two proposition we refer to Theorems 26 and 28 in [FGS04].

Proposition 13 (Existence of the asymptotic observable). Assume that Hypotheses (HO), (H1) and (H3) are satisfied. Fix $\beta<1$, and choose $\Sigma<\min \left(\Sigma_{\beta}, \Sigma_{\text {ion }}\right)$. Suppose that $f \in C_{0}^{\infty}(\mathbb{R})$ with $\operatorname{supp}(f) \subset(-\infty, \Sigma)$. Let $\gamma$, and $\chi_{\gamma}$ be as defined above, and let $\chi_{\gamma, t}$ be the operator of multiplication with $\chi_{\gamma}(|y| / t)$. Then, for $g \geq 0$ small enough (in order for (44) to hold true),

$$
W=s-\lim _{t \rightarrow \infty} e^{i H_{\text {mod }} t} f \mathrm{~d} \Gamma\left(\chi_{\gamma, t}\right) f e^{-i H_{\text {mod }} t}
$$

exists, $W=W^{*}$ and $W$ commutes with $H_{\text {mod. }}$ Here $f=f\left(H_{\text {mod }}\right)$.

Proposition 14 (Existence of $W_{+}$). Assume Hypotheses $(H O),(H 1)$ and $(H 3)$ are satisfied. Fix $\beta<1$ and choose $\Sigma<\min \left(\Sigma_{\beta}, \Sigma_{i o n}\right)$. Suppose that $f \in C_{0}^{\infty}(\mathbb{R})$ with $\operatorname{supp}(f) \subset(-\infty, \Sigma)$, and that $\chi_{\gamma}$ and $j_{t}$ are defined as described above. If $g \geq 0$ is so small that (44) holds, then

(i) the limit

$$
W_{+}=s-\lim _{t \rightarrow \infty} e^{i \tilde{H}_{m o d} t} f\left(\tilde{H}_{m o d}\right) \breve{\Gamma}\left(j_{t}\right) \mathrm{d} \Gamma\left(\chi_{\gamma, t}\right) f\left(H_{m o d}\right) e^{-i H_{m o d} t}
$$

exists, and $e^{-i \tilde{H}_{m o d} s} W_{+}=W_{+} e^{-i H_{m o d} s}$, for all $s \in \mathbb{R}$;

(ii) $\left(1 \otimes \chi_{\sim}(N=0)\right) W_{+}=0$;

(iii) $W=\tilde{\Omega}_{+} W_{+}$. 
4.4. Positivity of the asymptotic observable and asymptotic completeness. In this section we prove the positivity of the asymptotic observable $W$, restricted to the subspace of states orthogonal to wave packets of dressed atom states and not containing any soft bosons. We need the following lemma.

Lemma 15. Assume Hypotheses $(H 0),(H 1),(H 3)$. Fix $\beta<1$ and choose $\Sigma<\min \left(\Sigma_{\beta}\right.$, $\left.\Sigma_{\text {ion }}\right)$. Suppose, moreover, that $f \in C_{0}^{\infty}(\mathbb{R})$ and $\operatorname{supp}(f) \subset(-\infty, \Sigma)$. Put $a_{X}=$ $(1 / 2)(\nabla \omega \cdot(y-X)+(y-X) \cdot \nabla \omega)$, where $X$ is the position of the center of mass of the atom. Then, if $g$ is so small that (44) holds true, we have that

$$
f\left(H_{\text {mod }}\right)\left[i H_{\text {mod }}, \mathrm{d} \Gamma\left(a_{X}\right)\right] f\left(H_{\text {mod }}\right) \geq(1-\beta) f\left(H_{\text {mod }}\right) N f\left(H_{\text {mod }}\right)-C g f\left(H_{\text {mod }}\right)^{2}
$$

on the range of the projection $\Gamma\left(\chi_{i}\right)$.

This lemma follows from a straightforward estimate of the commutator $\left[H_{\text {mod }}, \mathrm{d} \Gamma\left(a_{X}\right)\right]$, from (44), (45), and from Lemma 21.

Theorem 16 (Positivity of the asymptotic observable). Assume that Hypotheses (HO)(H3) are satisfied. Fix $\beta<1 / 3$ and choose $\Sigma<\min \left(\Sigma_{\beta}, \Sigma_{i o n}\right)\left(\right.$ with $\Sigma_{\beta}$ and $\Sigma_{i o n}$ as in Lemma 1). Let the operator $W$ be defined as in Proposition 13 with supp $f \subset(-\infty, \Sigma)$. Then if $g>0$ is sufficiently small we can choose $\gamma>\beta$ in the definition of $W$ such that

$$
\langle\varphi, W \varphi\rangle \geq C\left\|f\left(H_{m o d}\right) \varphi\right\|^{2}, \quad \text { for all } \varphi \in \operatorname{Ran} P_{d a s}^{\perp} \Gamma\left(\chi_{i}\right) .
$$

Here $C$ is a positive constant depending on $g, \beta, \Sigma$, but independent of $\varphi$. In particular, if $\Delta \subset(-\infty, \Sigma)$ and then $f=1$ on $\Delta$, then

$$
\left.W\right|_{\operatorname{Ran} E_{\Delta}\left(H_{m o d}\right) \Gamma\left(\chi_{i}\right) P} \stackrel{\perp d a s}{\perp} \geq C>0 .
$$

Proof. Let $\mathcal{D}=D(\mathrm{~d} \Gamma(a)) \cap \operatorname{Ran} \Gamma\left(\chi_{i}\right) P_{\text {das }}^{\perp}$. Since $\mathcal{D}$ is dense in $\operatorname{Ran} \Gamma\left(\chi_{i}\right) P_{\text {das }}^{\perp}$, it is enough to prove that

$$
\langle\varphi, W \varphi\rangle \geq C\|f \varphi\|^{2}
$$

for every $\varphi \in \mathcal{D}$. As before, we use the notation $f=f\left(H_{\text {mod }}\right)$.

The first step consists in proving that there exists a constant $C$, depending only on $\Sigma$, such that, for every $\varphi \in \mathcal{D}$ and for every $\varepsilon>0$,

$$
\begin{aligned}
\left\langle\varphi_{t}, f \mathrm{~d} \Gamma\left(\chi_{\gamma, t}\right) f \varphi_{t}\right\rangle \geq & C\|f \varphi\|^{-2}\left[\frac{1-\beta}{t} \int_{0}^{t} \mathrm{~d} s\left\langle\varphi_{s}, f N f \varphi_{s}\right\rangle-(\gamma+\beta+\varepsilon)\right. \\
& \left.\times\left\langle\varphi_{t}, f N f \varphi_{t}\right\rangle\right]^{2}-C g\|f \varphi\|^{2}+o(1), \quad \text { as } \quad t \rightarrow \infty .
\end{aligned}
$$

This inequality follows from Lemma 15 by straightforward adaptations of arguments given in the proof of Theorem 27 in [FGS04]. Next, we observe that

$$
\frac{1}{t} \int_{0}^{t} \mathrm{~d} s\left\langle\varphi_{s}, f N f \varphi_{s}\right\rangle \geq\|f \varphi\|^{2}-\frac{1}{t} \int_{0}^{t}\left\langle\varphi_{s}, f P_{\Omega} f \varphi_{s}\right\rangle,
$$

where $P_{\Omega}$ denotes the orthogonal projection onto the Fock vacuum $\Omega$. The second term on the r.h.s. of the last equation can be written as an integral over fibers with fixed 
total momentum. Making use of the fact that $\varphi=P_{\text {das }}^{\perp} \varphi$ and of Fubini's Theorem to interchange the integration over $s$ and over $\Pi$, we obtain

$$
\frac{1}{t} \int_{0}^{t} \mathrm{~d} s\left\langle\varphi_{s}, f P_{\Omega} f \varphi_{s}\right\rangle=\int \mathrm{d} \Pi \frac{1}{t} \int_{0}^{t} \mathrm{~d} s\left\|P_{\Omega} f\left(H_{\bmod }(\Pi)\right) e^{-i H_{\bmod }(\Pi) s} P_{\psi_{\Pi}}^{\perp} \varphi(\Pi)\right\|^{2},
$$

where $P_{\psi_{\Pi}}=\left|\psi_{\Pi}\right\rangle\left\langle\psi_{\Pi}\right|$ is the orthogonal projection onto the dressed atom state $\psi_{\Pi}$, and $P_{\psi_{\Pi}}^{\perp}=1-P_{\psi_{\Pi}}$ is its orthogonal complement. For every fixed $\Pi$, the operator $P_{\Omega} f\left(H_{\text {mod }}(\Pi)\right)$ is compact on $L^{2}\left(\mathbb{R}^{3}, \mathrm{~d} x\right) \otimes \mathcal{F}$, because $\left\|e^{\alpha|x|} E_{\Sigma}\left(H_{\text {mod }}(\Pi)\right)\right\| \leq C$; (since $\Sigma<\Sigma_{\text {ion }}$, this follows from Lemma 1). By the continuity of the spectrum of $H_{\text {mod }}(\Pi)$ on $\operatorname{Ran} E_{\Sigma}\left(H_{\text {mod }}\right) P_{\psi_{\Pi}}^{\perp} \Gamma\left(\chi_{i}\right)$ (see Corollary 6), and the RAGE Theorem (see, for example, [RS79]), it follows that

$$
\frac{1}{t} \int_{0}^{t} \mathrm{~d} s\left\|P_{\Omega} f\left(H_{\bmod }(\Pi)\right) e^{-i H_{\bmod }(\Pi) s} P_{\psi_{\Pi}}^{\perp} \varphi(\Pi)\right\|^{2} \rightarrow 0,
$$

as $t \rightarrow \infty$, pointwise in $\Pi$. Using Lebesgue's Dominated Convergence Theorem, we conclude that

$$
\frac{1}{t} \int_{0}^{t} \mathrm{~d} s\left\langle\varphi_{s}, f P_{\Omega} f \varphi_{s}\right\rangle \rightarrow 0
$$

as $t \rightarrow \infty$. From (155) we obtain

$$
\frac{1}{t} \int_{0}^{t} \mathrm{~d} s\left\langle\varphi_{s}, f N f \varphi_{s}\right\rangle \geq \frac{\|f \varphi\|^{2}}{2}
$$

for $t$ large enough, where we can assume $\|f \varphi\| \neq 0$ without loss of generality. Equations (158) and (159) allow us to apply Lemma 17, with $h_{1}(s)=\left\langle\varphi_{s}, f N f \varphi_{s}\right\rangle$ and $h_{2}(s)=\left\langle\varphi_{s}, f P_{\Omega} f \varphi_{s}\right\rangle$ (it is easy to check that $h_{1}$ and $h_{2}$ are bounded and continuous). We conclude that there exists a sequence $\left\{t_{n}\right\}_{n \geq 0}$ with $t_{n} \rightarrow \infty$, as $n \rightarrow \infty$, such that

$$
\begin{aligned}
\frac{1}{t_{n}} \int_{0}^{t_{n}} \mathrm{~d} s\left\langle\varphi_{s}, f N f \varphi_{s}\right\rangle & \geq(1-\varepsilon)\left\langle\varphi_{t_{n}}, f N f \varphi_{t_{n}}\right\rangle, \quad \text { and } \\
\left\langle\varphi_{t_{n}}, f P_{\Omega} f \varphi_{t_{n}}\right\rangle & \rightarrow 0, \quad \text { as } n \rightarrow \infty .
\end{aligned}
$$

From (154) we infer that

$$
\begin{aligned}
\left\langle\varphi_{t_{n}}, f \mathrm{~d} \Gamma\left(\chi_{\gamma, t_{n}}\right) f \varphi_{t_{n}}\right\rangle \geq & C\|f \varphi\|^{-2}(1-2 \beta-\gamma-2 \varepsilon)^{2}\left\langle\varphi_{t_{n}}, f N f \varphi_{t_{n}}\right\rangle^{2} \\
& -C g\|f \varphi\|^{2}+o(1),
\end{aligned}
$$

as $n \rightarrow \infty$. Choosing $\gamma-\beta$ and $\varepsilon>0$ sufficiently small, we conclude that

$$
\begin{aligned}
\left\langle\varphi_{t_{n}}, f \mathrm{~d} \Gamma\left(\chi_{\gamma, t_{n}}\right) f \varphi_{t_{n}}\right\rangle \geq & C \frac{(1-3 \beta)^{2}}{2}\|f \varphi\|^{-2}\left\langle\varphi_{t_{n}}, f N f \varphi_{t_{n}}\right\rangle^{2}-C g\|f \varphi\|^{2}+o(1) \\
\geq & C \frac{(1-3 \beta)^{2}}{2}\left(\|f \varphi\|^{2}-2\left\langle\varphi_{t_{n}}, f P_{\Omega} f \varphi_{t_{n}}\right\rangle\right) \\
& -C g\|f \varphi\|^{2}+o(1),
\end{aligned}
$$


as $n \rightarrow \infty$. Hence, by (160), there are constants $C_{1}>0$ and $C_{2}<\infty$, depending only on $\Sigma$, such that

$$
\left\langle\varphi_{t_{n}}, f \mathrm{~d} \Gamma\left(\chi_{\gamma, t_{n}}\right) f \varphi_{t_{n}}\right\rangle \geq C_{1}\left(1-3 \beta-C_{2} g\right)^{2}\|f \varphi\|^{2}+o(1),
$$

as $n \rightarrow \infty$. If $\beta<1 / 3$ and $g$ is small enough, we arrive at (151) by taking the limit $n \rightarrow \infty$. (Since we already know that the limit defining $W$ exists, it is enough to prove its positivity on some arbitrary subsequence!)

Lemma 17. Suppose $h_{1}$ and $h_{2}$ are positive, continuous, bounded functions on $\mathbb{R}$, such that

$$
m_{1}(t):=\frac{1}{t} \int_{0}^{t} \mathrm{~d} s h_{1}(s) \geq C>0
$$

for all $t>0$ large enough, and

$$
m_{2}(t):=\frac{1}{t} \int_{0}^{t} \mathrm{~d} s h_{2}(s) \rightarrow 0 \text { as } t \rightarrow \infty .
$$

Then, for every $\delta>0$, there exists a sequence $\left\{t_{n}\right\}_{n \geq 0}$, with $t_{n} \rightarrow \infty$, as $n \rightarrow \infty$, such that

$$
m_{1}\left(t_{n}\right) \geq \frac{1}{1+\delta} h_{1}\left(t_{n}\right)
$$

and

$$
h_{2}\left(t_{n}\right) \rightarrow 0 \quad \text { as } n \rightarrow \infty
$$

Proof. Define the sets

$$
S_{T}:=\left\{t \in[0, T]: m_{1}(t)<\frac{1}{1+\delta} h_{1}(t)\right\}, \quad \text { for some } \delta>0 .
$$

By the continuity of $h_{1}(t)$ and $m_{1}(t)$ the sets $S_{T}$ are measurable (with respect to Lebesgue measure on $\mathbb{R}$ ), for all $T$. Denote by $\mu(A)$ the Lebesgue measure of a measurable set $A \subset \mathbb{R}$. We show that

$$
\liminf _{T \rightarrow \infty} \frac{\mu\left(S_{T}\right)}{T}<1
$$

In fact, if (168) were false, then (since $\mu\left(S_{T}\right) / T \leq 1$ for all $T \geq 0$ )

$$
\lim _{T \rightarrow \infty} \frac{\mu\left(S_{T}\right)}{T}=1
$$

and hence, for arbitrary $\varepsilon>0$, we could find a $T_{0}$ such that

$$
\frac{\mu\left(S_{T}\right)}{T} \geq 1-\varepsilon,
$$


for all $T>T_{0}$. This would imply that

$$
\begin{aligned}
m_{1}(T) & =\frac{1}{T} \int_{0}^{T} \mathrm{~d} s h_{1}(s) \geq \frac{1}{T} \int_{S_{T}} \mathrm{~d} s h_{1}(s) \\
& \geq \frac{1+\delta}{T} \int_{S_{T}} \mathrm{~d} s m_{1}(s) \geq \frac{1+\delta}{T} \int_{0}^{T} \mathrm{~d} s m_{1}(s)-\frac{\mu\left(S_{T}^{c}\right)}{T}\left\|m_{1}\right\|_{\infty},
\end{aligned}
$$

where $\left\|m_{1}\right\|_{\infty}$ denotes the supremum of the bounded function $m_{1}$ and $S_{T}^{c}=[0, T] \backslash S_{T}$ is the complement of $S_{T}$ inside $[0, T]$. Hence, we find

$$
m_{1}(T) \geq \frac{1+\delta}{T} \int_{0}^{T} \mathrm{~d} s m_{1}(s)-\varepsilon\left\|m_{1}\right\|_{\infty}
$$

for every $T \geq T_{0}$. Put $\widetilde{m}_{1}(T):=(1 / T) \int_{0}^{T} \mathrm{~d} s m_{1}(s)$. Then we have

$$
\frac{d}{d T} \log \widetilde{m}_{1}(T)=\frac{\widetilde{m}_{1}^{\prime}(T)}{\widetilde{m}_{1}(T)}=\frac{1}{T}\left(\frac{m_{1}(T)}{\widetilde{m}_{1}(T)}-1\right) \geq \frac{1}{T}\left(\delta-\frac{\varepsilon\left\|m_{1}\right\|_{\infty}}{\widetilde{m}_{1}(T)}\right) .
$$

By the assumption that $m_{1}(T) \geq C$ for all $T$ large enough, we have $\tilde{m}_{1}(T) \geq C$, and thus, choosing $\varepsilon<C \delta / 2\left\|m_{1}\right\|_{\infty}$, we find

$$
\frac{d}{d T} \log \widetilde{m}_{1}(T) \geq \frac{\delta}{2 T}
$$

for all $T$ large enough. This contradicts the boundedness of $\widetilde{m}_{1}(T)$ (which follows from the boundedness of $m_{1}(T)$ ). This proves (168), and implies that there exist $\varepsilon>0$ and a sequence $\left\{T_{m}\right\}_{m \geq 0}$ converging to infinity such that

$$
\frac{\mu\left(S_{T_{m}}\right)}{T_{m}} \leq 1-\varepsilon
$$

for all $m \geq 0$. Hence $\mu\left(S_{T_{m}}^{c}\right) \geq \varepsilon T_{m}$, for all $m$. Next, we show that there exists a sequence $\left\{t_{n}\right\}_{n \geq 0}$, with $t_{n} \rightarrow \infty$ as $n \rightarrow \infty$, such that $t_{n} \in \cup_{m \geq 0} S_{T_{m}}^{c}$, for all $n \geq 0$, and

$$
h_{2}\left(t_{n}\right) \rightarrow 0
$$

as $n \rightarrow \infty$. Since, for all $n \geq 0, t_{n} \in S_{T_{m}}^{c}$, for some $m \in \mathbb{N}$, the sequence $t_{n}$ automatically satisfies (166). Thus the lemma follows if we can prove (174). To this end we argue again by contradiction. If there were no sequence $\left\{t_{n}\right\}_{n \geq 0} \in \cup_{m \geq 0} S_{T_{m}}^{c}$ satisfying (174) then there would exist $\tau$ and $\alpha>0$ such that $h_{2}(t) \geq \alpha$, for all $t \in \cup_{m \geq 0} S_{T_{m}}^{c} \cap[\tau, \infty)$. But then, for an arbitrary $m \in \mathbb{N}$ with $T_{m} \geq \tau$,

$$
\begin{aligned}
\frac{1}{T_{m}} \int_{0}^{T_{m}} \mathrm{~d} s h_{2}(s) & \geq \frac{1}{T_{m}} \int_{S_{T_{m}}^{c} \cap\left[\tau, T_{m}\right]} \mathrm{d} s h_{2}(s) \geq \alpha \frac{\mu\left(S_{T_{m}}^{c} \cap\left[\tau, T_{m}\right]\right)}{T_{m}} \\
& \geq \alpha \frac{\mu\left(S_{T_{m}}^{c}\right)}{T_{m}}-\frac{\alpha \tau}{T_{m}} \geq \alpha \varepsilon-\frac{\alpha \tau}{T_{m}}
\end{aligned}
$$

for all $m \in \mathbb{N}$ with $T_{m} \geq \tau$. Taking $m \rightarrow \infty$, this contradicts the assumption (165). 
4.5. Asymptotic completeness. Using the positivity of the asymptotic observable $W$, we can complete the proof of asymptotic completeness for the Hamiltonian $H_{\text {mod }}$. Our proof is based on induction in the energy. The following simple lemma is useful.

Lemma 18. Assume that Hypotheses (HO)-(H3) are satisfied. Fix $\beta<1$ and choose $\Sigma<\min \left(\Sigma_{\beta}, \Sigma_{i o n}\right)$. The wave operators $\tilde{\Omega}_{+}$and $\Omega_{+}$are defined as in Lemma 10 and in Theorem 8, respectively. Suppose that $\operatorname{Ran} \Omega_{+} \supset E_{\eta}\left(H_{\text {mod }}\right) \mathcal{H}$, for some $\eta<\Sigma$. Then, for every $\varphi \in \operatorname{Ran} E_{\Sigma}\left(\tilde{H}_{\text {mod }}\right)$, there exists $\psi \in \operatorname{Ran} E_{\Sigma}\left(\tilde{H}_{m o d}\right)$ such that

$$
\tilde{\Omega}_{+}\left(E_{\eta}\left(H_{m o d}\right) \otimes 1\right) \varphi=\Omega_{+} \psi .
$$

If $\Delta \subset(-\infty, \Sigma)$ and $\varphi \in E_{\Delta}\left(\tilde{H}_{m o d}\right) \tilde{\mathcal{H}}$ then $\psi \in E_{\Delta}\left(\tilde{H}_{\text {mod }}\right) \tilde{\mathcal{H}}$.

The interpretation of this lemma is simple: If we know that asymptotic completeness holds for vectors with energy lower than $\eta$, then it continues to be true if we add asymptotically free photons to these vectors (no matter what the total energy of the new state is). For the proof of this lemma we refer to Lemma 20 of [FGS04]. Using this lemma we can prove asymptotic completeness for $H_{\text {mod }}$; the proof is similar to the proof of Theorem 19 in [FGS04]. We repeat it here, because it is very short, and because it explains the ideas behind all the tools introduced in Sect. 4.

Theorem 19. Assume that Hypotheses (HO)-(H3) are satisfied. Fix $\beta<1 / 3$ and choose $\Sigma<\min \left(\Sigma_{\beta}, \Sigma_{i o n}\right)$; (with $\Sigma_{\beta}$ and $\Sigma_{i o n}$ defined as in Lemma 1$)$. If $g>0$ is sufficiently small, then

$$
\operatorname{Ran} \Omega_{+} \supset E_{(-\infty, \Sigma)}\left(H_{\text {mod }}\right) \mathcal{H} .
$$

Proof. The proof is by induction in energy steps of size $m=\sigma / 2$. We show that

$$
\operatorname{Ran} \Omega_{+} \supset E_{(-\infty, \Sigma-k m)}\left(H_{\text {mod }}\right) \mathcal{H}
$$

holds for $k=0$, by proving this claim for all $k \in\{0,1,2, \ldots\}$. Since $H_{\text {mod }}$ is bounded below, (176) is obviously correct for $k$ large enough. Assuming that (176) holds for $k=n+1$, we now prove it for $k=n$. Since Ran $\Omega_{+}$is closed (by Theorem 8) and since $\operatorname{Ran} \Omega_{+} \supset \mathcal{H}_{\text {das }}$, it suffices to prove that

$$
\operatorname{Ran} \Omega_{+} \supset P_{\text {das }}^{\perp} \Gamma\left(\chi_{i}\right) E_{\Delta}\left(H_{\text {mod }}\right) \mathcal{H},
$$

for $\Delta=\left(\inf \sigma\left(H_{g=0}\right)-1, \Sigma-n m\right)$. Here we use Lemma 11. Fix $\widetilde{\Sigma}$ with $\Sigma<$ $\widetilde{\Sigma}<\min \left(\Sigma_{\beta}, \Sigma_{\text {ion }}\right)$ and choose $f \in C_{0}^{\infty}(\mathbb{R})$ real-valued, with $f \equiv 1$ on $\Delta$ and $\operatorname{supp}(f) \subset(-\infty, \widetilde{\Sigma})$. We define the asymptotic observable $W$ in terms of $f$, as in Proposition 13. By Theorem 16, the operator $\Gamma\left(\chi_{i}\right) P_{\mathrm{das}}^{\perp} W P_{\mathrm{das}}^{\perp} \Gamma\left(\chi_{i}\right)$ is strictly positive on $P_{\text {das }}^{\perp} \Gamma\left(\chi_{i}\right) E_{\Delta}\left(H_{\text {mod }}\right) \mathcal{H}$, and hence onto, if $g$ is small enough. Given $\psi$ in this space, we can therefore find a vector $\varphi=P_{\text {das }}^{\perp} \Gamma\left(\chi_{i}\right) \varphi$ such that

$$
P_{\text {das }}^{\perp} \Gamma\left(\chi_{i}\right) W \varphi=\psi
$$

By Proposition $14, W \varphi=\tilde{\Omega}_{+} W_{+} \varphi$ and $W_{+} \varphi=E_{\Sigma-n m}\left(\tilde{H}_{\text {mod }}\right) W_{+} \varphi$. Furthermore, by part (ii) of Proposition 14, $W_{+} \varphi$ has at least one boson in the outer Fock space, and thus an energy of at most $\Sigma-(n+1) m$ in the inner one. That is,

$$
W_{+} \varphi=\left[E_{\Sigma-(n+1) m}\left(H_{\mathrm{mod}}\right) \otimes 1\right] W_{+} \varphi .
$$


Hence we can use the induction hypothesis Ran $\Omega_{+} \supset E_{\Sigma-(n+1) m}\left(H_{\text {mod }}\right) \mathcal{H}$. By Lemma 18, it follows that $\tilde{\Omega}_{+} W_{+} \varphi=\Omega_{+} \gamma$ for some $\gamma \in E_{\Delta}\left(\tilde{H}_{\text {mod }}\right) \mathcal{H}$. We conclude that

$$
\begin{aligned}
\psi & =\Gamma\left(\chi_{i}\right) P_{\text {das }}^{\perp} \Omega_{+} \gamma \\
& =\Gamma\left(\chi_{i}\right) \Omega_{+}\left(1 \otimes P_{\Omega}^{\perp}\right) \gamma \\
& =\Omega_{+}\left(\Gamma\left(\chi_{i}\right) \otimes \Gamma\left(\chi_{i}\right) P_{\Omega}^{\perp}\right) \gamma,
\end{aligned}
$$

where $P_{\Omega}^{\perp}$ is the projection onto the orthogonal complement of the vacuum. This proves the theorem.

\section{A. Fock Space and Second Quantization}

Let $\mathfrak{h}$ be a complex Hilbert space, and let $\otimes_{s}^{n} \mathfrak{h}$ denote the $n$-fold symmetric tensor product of $\mathfrak{h}$. Then the bosonic Fock space over $\mathfrak{h}$,

$$
\mathcal{F}=\mathcal{F}(\mathfrak{h})=\bigoplus_{n \geq 0} \mathfrak{h}^{\otimes_{s} n},
$$

is the space of sequences $\varphi=\left(\varphi_{n}\right)_{n \geq 0}$, with $\varphi_{0} \in \mathbb{C}, \varphi_{n} \in \otimes_{S}^{n} \mathfrak{h}$, and with the scalar product given by

$$
\langle\varphi, \psi\rangle:=\sum_{n \geq 0}\left(\varphi_{n}, \psi_{n}\right),
$$

where $\left(\varphi_{n}, \psi_{n}\right)$ denotes the inner product in $\otimes_{s}^{n} \mathfrak{h}$. The vector $\Omega=(1,0, \ldots) \in \mathcal{F}$ is called the vacuum. By $\mathcal{F}_{0} \subset \mathcal{F}$ we denote the dense subspace of vectors $\varphi$ for which $\varphi_{n}=0$, for all but finitely many $n$. The number operator $N$ is defined by $(N \varphi)_{n}=n \varphi_{n}$.

A.1. Creation- and annihilation operators. The creation operator $a^{*}(h), h \in \mathfrak{h}$, is defined on $\mathfrak{h}^{\otimes_{s} n-1}$ by

$$
a^{*}(h) \varphi=\sqrt{n} S(h \otimes \varphi), \quad \text { for } \varphi \in \mathfrak{h}^{\otimes_{s} n-1},
$$

and extended by linearity to $\mathcal{F}_{0}$. Here $S$ denotes the orthogonal projection onto the symmetric subspace $\otimes_{s}^{n} \mathfrak{h} \subset \otimes^{n} \mathfrak{h}$. The annihilation operator $a(h)$ is the adjoint of $a^{*}(h)$. Creation- and annihilation operators satisfy the canonical commutation relations (CCR)

$$
\left[a(g), a^{*}(h)\right]=(g, h), \quad\left[a^{\#}(g), a^{\#}(h)\right]=0 .
$$

In particular, $\left[a(h), a^{*}(h)\right]=\|h\|^{2}$, which implies that the graph norms associated with the closable operators $a(h)$ and $a^{*}(h)$ are equivalent. It follows that the closures of $a(h)$ and $a^{*}(h)$ have the same domain. On this common domain we define the self-adjoint operator

$$
\phi(h)=a(h)+a^{*}(h) .
$$

The creation- and annihilation operators, and thus $\phi(h)$, are bounded relative to the square root of the number operator:

$$
\left\|a^{\#}(h)(N+1)^{-1 / 2}\right\| \leq\|h\| .
$$

More generally, for any $p \in \mathbb{R}$ and any integer $n$,

$$
\left\|(N+1)^{p} a^{\#}\left(h_{1}\right) \ldots a^{\#}\left(h_{n}\right)(N+1)^{-p-n / 2}\right\| \leq C_{n, p}\left\|h_{1}\right\| \cdot \ldots \cdot\left\|h_{n}\right\| .
$$


A.2. The functor $\Gamma$. Let $\mathfrak{h}_{1}$ and $\mathfrak{h}_{2}$ be two Hilbert spaces and let $b \in \mathbf{B}\left(\mathfrak{h}_{1}, \mathfrak{h}_{2}\right)$. We define $\Gamma(b): \mathcal{F}\left(\mathfrak{h}_{1}\right) \rightarrow \mathcal{F}\left(\mathfrak{h}_{2}\right)$ by

$$
\Gamma(b)\left\lceil\otimes_{s}^{n} \mathfrak{h}_{1}=b \otimes \cdots \otimes b .\right.
$$

In general $\Gamma(b)$ is unbounded; but if $\|b\| \leq 1$ then $\|\Gamma(b)\| \leq 1$. From the definition of $a^{*}(h)$ it easily follows that

$$
\begin{array}{rlrl}
\Gamma(b) a^{*}(h) & =a^{*}(b h) \Gamma(b), & h \in \mathfrak{h}_{1}, \\
\Gamma(b) a\left(b^{*} h\right) & =a(h) \Gamma(b), \quad h \in \mathfrak{h}_{2} .
\end{array}
$$

If $b^{*} b=1$ on $\mathfrak{h}_{1}$ then these equations imply that

$$
\begin{aligned}
\Gamma(b) a(h) & =a(b h) \Gamma(b), & & h \in \mathfrak{h}_{1}, \\
\Gamma(b) \phi(h) & =\phi(b h) \Gamma(b), & & h \in \mathfrak{h}_{1} .
\end{aligned}
$$

A.3. The operator $\mathrm{d} \Gamma(b)$. Let $b$ be an operator on $\mathfrak{h}$. Then $\mathrm{d} \Gamma(b): \mathcal{F}(\mathfrak{h}) \rightarrow \mathcal{F}(\mathfrak{h})$ is defined by

$$
\mathrm{d} \Gamma(b)\left\lceil\otimes_{s}^{n} \mathfrak{h}=\sum_{i=1}^{n}(1 \otimes \ldots b \otimes \ldots 1) .\right.
$$

For example $N=\mathrm{d} \Gamma(1)$. From the definition of $a^{*}(h)$ we infer that

$$
\left[\mathrm{d} \Gamma(b), a^{*}(h)\right]=a^{*}(b h) \quad[\mathrm{d} \Gamma(b), a(h)]=-a\left(b^{*} h\right),
$$

and, if $b=b^{*}$,

$$
i[\mathrm{~d} \Gamma(b), \phi(h)]=\phi(i b h) .
$$

Note that $\left\|\mathrm{d} \Gamma(b)(N+1)^{-1}\right\| \leq\|b\|$.

A.4. The tensor product of two Fock spaces. Let $\mathfrak{h}_{1}$ and $\mathfrak{h}_{2}$ be two Hilbert spaces. We define a linear operator $U: \mathcal{F}\left(\mathfrak{h}_{1} \oplus \mathfrak{h}_{2}\right) \rightarrow \mathcal{F}\left(\mathfrak{h}_{1}\right) \otimes \mathcal{F}\left(\mathfrak{h}_{2}\right)$ by

$$
\begin{aligned}
U \Omega & =\Omega \otimes \Omega, \\
U a^{*}(h) & =\left[a^{*}\left(h_{(0)}\right) \otimes 1+1 \otimes a^{*}\left(h_{(\infty)}\right)\right] U \quad \text { for } h=\left(h_{(0)}, h_{(\infty)}\right) \in \mathfrak{h}_{1} \oplus \mathfrak{h}_{2} .
\end{aligned}
$$

This defines $U$ on finite linear combinations of vectors of the form $a^{*}\left(h_{1}\right) \ldots a^{*}\left(h_{n}\right) \Omega$. From the CCRs it follows that $U$ is isometric. Its closure is isometric and onto, hence unitary.

A.5. Factorizing Fock space in a tensor product. Suppose $j_{0}$ and $j_{\infty}$ are linear operators on $\mathfrak{h}$ and $j: \mathfrak{h} \rightarrow \mathfrak{h} \oplus \mathfrak{h}$ is defined by $j h=\left(j_{0} h, j_{\infty} h\right), h \in \mathfrak{h}$. Then $j^{*}\left(h_{1}, h_{2}\right)=$ $j_{0}^{*} h_{1}+j_{\infty}^{*} h_{2}$ and consequently $j^{*} j=j_{0}^{*} j_{0}+j_{\infty}^{*} j_{\infty}$. We define

$$
\breve{\Gamma}(j)=U \Gamma(j): \mathcal{F} \rightarrow \mathcal{F} \otimes \mathcal{F},
$$

where $\Gamma(j)$ is as defined in Sect. A.2. It follows that $\breve{\Gamma}(j)^{*} \breve{\Gamma}(j)=\Gamma\left(j^{*} j\right)$ which is the identity if $j^{*} j=1$. In this case

$$
\begin{aligned}
\breve{\Gamma}(j) a^{\#}(h) & =\left[a^{\#}\left(j_{0} h\right) \otimes 1+1 \otimes a^{\#}\left(j_{\infty} h\right)\right] \breve{\Gamma}(j), \\
\breve{\Gamma}(j) \phi(h) & =\left[\phi\left(j_{0} h\right) \otimes 1+1 \otimes \phi\left(j_{\infty} h\right)\right] \breve{\Gamma}(j) .
\end{aligned}
$$


A.6. The "Scattering Identification". We define the scattering identification $I: \mathcal{F} \otimes$ $\mathcal{F} \rightarrow \mathcal{F}$ by

$$
\begin{aligned}
I(\varphi \otimes \Omega) & =\varphi, \\
I \varphi \otimes a^{*}\left(h_{1}\right) \cdots a^{*}\left(h_{n}\right) \Omega & =a^{*}\left(h_{1}\right) \cdots a^{*}\left(h_{n}\right) \varphi, \quad \varphi \in \mathcal{F}_{0},
\end{aligned}
$$

and extend it by linearity to $\mathcal{F}_{0} \otimes \mathcal{F}_{0}$. (Note that this definition is symmetric with respect to the two factors in the tensor product.) There is a second characterization of $I$ which can be useful. Let $\iota: \mathfrak{h} \oplus \mathfrak{h} \rightarrow \mathfrak{h}$ be defined by $\iota\left(h_{(0)}, h_{(\infty)}\right)=h_{(0)}+h_{(\infty)}$. Then $I=\Gamma(\iota) U^{*}$, with $U$ as above. Since $\|\iota\|=\sqrt{2}$, the operator $I$ is unbounded, but it can be proved that $I(N+1)^{-k} \otimes \chi(N \leq k)$ is bounded, for any $k \geq 1$.

\section{B. Bounds on the Interaction}

In this section we review standard estimates that are used throughout this paper to bound the interaction.

Lemma 20. Let $L_{\omega}^{2}\left(\mathbb{R}^{3}\right):=L^{2}\left(\mathbb{R}^{3},(1+1 /|k|) d k\right)$ and let $h \in L_{\omega}^{2}\left(\mathbb{R}^{3}\right)$. Then

$$
\begin{aligned}
\|a(h) \varphi\| & \leq\left(\int d k|h(k)|^{2} /|k|\right)^{1 / 2}\left\|\mathrm{~d} \Gamma(|k|)^{1 / 2} \varphi\right\|, \\
\left\|a^{*}(h) \varphi\right\| & \leq\|h\|_{\omega}\left\|(\mathrm{d} \Gamma(|k|)+1)^{1 / 2} \varphi\right\|, \\
\|\phi(h) \varphi\| & \leq \sqrt{2}\|h\|_{\omega}\left\|(\mathrm{d} \Gamma(|k|)+1)^{1 / 2} \varphi\right\|, \\
\pm \phi(h) & \leq \alpha \mathrm{d} \Gamma(|k|)+\frac{1}{\alpha} \int d k \frac{|h(k)|^{2}}{|k|}, \quad \alpha>0,
\end{aligned}
$$

where $\|h\|_{\omega}^{2}=\int d k(1+1 /|k|)|h(k)|^{2}$.

The next lemma is used to control the factor $\phi\left(i a F_{x}\right)$ appearing in the commutators of Sect. 3.3.

Lemma 21. Assume Hypothesis $(H O)-(H 1)$. Let $a=(1 / 2)(\hat{k} \cdot y+y \hat{k})$ with $\hat{k}=k /|k|$ and choose $\Sigma<\Sigma_{\text {ion. }}$. Then there exists $C_{\Sigma}<\infty$ such that

$$
\left\|\phi\left(i a F_{x}\right) E_{\Sigma}\left(H_{g}(\Pi)\right)\right\| \leq C_{\Sigma}
$$

with $F_{x}$ as in Eq. (32). For $a_{X}:=(1 / 2)(\hat{k} \cdot(y-X)+(y-X) \cdot \hat{k})$,

$$
\left\|\phi\left(i a_{X} G_{X, x}\right) E_{\Sigma}\left(H_{g}\right)\right\| \leq C_{\Sigma}
$$

where $G_{X, x}(k)=e^{-i k \cdot X} F_{X}(k)$; (see Eqs. (31), (32)).

Proof. Note that

$$
\begin{aligned}
\left(a F_{x}\right)(k)= & \left(i \hat{k} \cdot \nabla_{k}+2 /|k|\right)\left(e^{-i \lambda_{e} k \cdot x} \kappa_{e}(k)+e^{i \lambda_{n} k \cdot x} \kappa_{n}(k)\right) \\
= & e^{-i \lambda_{e} k \cdot x}\left(\lambda_{e} x \cdot \hat{k} \kappa_{e}(k)+i \hat{k} \cdot \nabla \kappa_{e}(k)+2 /|k| \kappa_{e}(k)\right) \\
& +e^{i \lambda_{n} k \cdot x}\left(-\lambda_{n} x \cdot \hat{k} \kappa_{n}(k)+i \hat{k} \cdot \nabla \kappa_{n}(k)+2 /|k| \kappa_{n}(k)\right) .
\end{aligned}
$$

Equation (187) follows from Lemma 20, because $e^{\alpha|x|} E_{\Sigma}\left(H_{g}(\Pi)\right)$ is bounded (see Lemma 1) and from Hypothesis (H1). Equation (188) follows from (187) because

$$
\left(a_{X} G_{X, x}\right)(k)=e^{-i X \cdot k}\left(a F_{X}\right)(k) .
$$




\section{References}

[AGG05] Amour, L., Grébert, B., Guillot, J.C.: The dressed mobile atoms and ions. J. Math. Pures Appl. 86(3), 177-200 (2006)

[BFP05] Bach, V., Fröhlich, J., Pizzo, A.: Infrared-finite algorithms in QED: I. The groundstate of an atom interacting with the quantized radiation field. Commun. Math. Phys. 264(1), 145-165 (2006)

[BFS98] Bach, V., Fröhlich, J., Sigal, I.M.: Quantum electrodynamics of confined nonrelativistic particles. Adv. Math. 137(2), 299-395 (1998)

[BFSS99] Bach, V., Fröhlich, J., Sigal, I.M., Soffer, A.: Positive commutators and spectrum of nonrelativistic QED. Commun. Math. Phys. 207(3), 557-587 (1999)

[DG99] Dereziński, J., Gérard, C.: Asymptotic completeness in quantum field theory. Massive Pauli-Fierz Hamiltonians. Rev. Math. Phys. 11(4), 383-450 (1999)

[Frö73] Fröhlich, J.: On the infrared problem in a model of scalar electrons and massless, scalar bosons. Ann. Inst. H. Poincaré, Sect. A XIX(1), 1-103 (1973)

[Frö74] Fröhlich, J.: Existence of dressed one-electron states in a class of persistent models. Fortschr. Phys. 22, 159-198 (1974)

[FGS00] Fröhlich, J., Griesemer, M., Schlein, B.: Asymptotic electromagnetic fields in models of quantum-mechanical matter interacting with the quantized radiation field. Adv. Math. 164(2), 349-398 (2001)

[FGS02] Fröhlich, J., Griesemer, M., Schlein, B.: Asymptotic completeness for Rayleigh scattering. Ann. Henri Poincaré 3, 107-170 (2002)

[FGS04] Fröhlich, J., Griesemer, M., Schlein, B.: Asymptotic completeness for Compton Scattering. Commun. Math. Phys. 252, 415-176 (2004)

[FGSi05] Fröhlich, J., Griesemer, M., Sigal, I.M.: Mourre estimate and spectral theory for the standard model of non-relativistic QED. Preprint arXiv: math-ph/0611013

[Gr04] Griesemer, M.: Exponential decay and ionization thresholds in non-relativistic quantum electrodynamics. J. Funct. Anal. 210(3), 321-340 (2004)

[GLL01] Griesemer, M., Lieb, E.H., Loss, M.: Ground states in non-relativistic quantum electrodynamics. Invent. Math. 145(3), 557-595 (2001)

[LL03] Lieb, E.H., Loss, M.: Existence of atoms and molecules in non-relativistic quantum electrodynamics. Adv. Theor. Math. Phys. 7(4), 1-54 (2003)

[HS95] Hübner, M., Spohn, H.: Spectral properties of the spin-boson Hamiltonian. Ann. Inst. H. Poincaré 62(3), 289-323 (1995)

[Pi03] Pizzo, A.: One-particle (improper) states in Nelson's massless model. Ann. Henri Poincaré, 4(3), 439-486 (2003)

[RS79] Reed, M., Simon, B.: Methods of modern mathematical physics: Scattering Theory. Volume 3, New York: Academic Press, 1979

[RS78] Reed, M., Simon, B.: Methods of modern mathematical physics: Analysis of Operators. Volume 4, New York: Academic Press, 1978

[Sk98] Skibsted, E.: Spectral analysis of $N$-body systems coupled to a bosonic field. Rev. Math. Phys. 10(7), 989-1026 (1998)

[Sp97] Spohn, H.: Asymptotic completeness for Rayleigh scattering. J. Math. Phys. 38(5), 2281-2296 (1997)

Communicated by H.-T. Yau 